\title{
A High Fidelity Mixed Reality System for Remote Collaboration
}

by

\author{
Stephen Thompson
}

\author{
A thesis \\ submitted to the Victoria University of Wellington \\ in fulfilment of the \\ requirements for the degree of \\ Master of Science \\ in Computer Graphics.
}

Victoria University of Wellington

2020 



\begin{abstract}
This thesis presents a novel system for enabling remote collaboration within a mixed reality environment. Since the increase of virtual and augmented reality headsets, there has been increased interest in improving remote collaboration. Systems have been proposed to use 3D geometry or $360^{\circ}$ video for providing remotely collaborating users with a view of the local, real-world environment. However, many systems provide limited interactions in the local environment and target using coupled views of all users, rather than simulating face-to-face interactions, or use virtual environments for the remote user, losing visual realism.

The presented system enables a user situated in a remote location to join a local user to collaborate on a task. An omni-directional camera is streamed to the remote user in real-time to provide a live view of the local space. The $360^{\circ}$ video is also used to provide believable lighting when compositing virtual objects into the real-world. Remote users are displayed to local users as an abstracted avatar to provide basic body gestures and social presence. Voice chat is also provided for verbal communication.

The system has been evaluated for technical performance and user experience. The evaluation found the performance of the system was suitable for real-time collaboration. Remote and local users were also found to have similar satisfaction with the system, experiencing high levels of presence, social presence and tele-presence. Shared cinematic and remote presentations are suggested as possible applications to guide further development of the system.
\end{abstract}




\section{Acknowledgments}

I wish to thank Dr Taehyun Rhee and Dr Andrew Chalmers for their supervision during the completion of this thesis, and for TEC New Zealand for funding the research through the Entrepreneurial University Program. I would also like to thank Dr Daniel Medeiros for his suggestions and help with the running of the user evaluation; Ian Loh, Lohit Petikam and the rest of the staff and students at the Computational Media Innovation Centre (CMIC) of Victoria University of Wellington for their help, suggestions and encouragement; and to my family for their support during the Masters. 


\section{List of Publications}

RHEE, T., THOMPSON, S., MEDEIROS, .D, DOS ANJOS, R. CHALMERS, A., Augmented Virtual Teleportation for High-Fidelity Telecollaboration. IEEE Transactions on Visualization and Computer Graphics (special issues for IEEE VR), accepted [Journal]

The following publications were related but not part of this thesis.

THOMPSON, S., CHALMERS, A., RHEE, T., Real-time Underwater Caustics for Mixed Reality $360^{\circ}$ Videos. IEEE VR 2019 [Poster]

THOMPSON, S., CHALMERS, A., RHEE, T., Real-Time Mixed Reality Rendering for Underwater $360^{\circ}$ Videos. ISMAR 2019 [Conference Paper]

THOMPSON, S., CHALMERS, A., MEDEIROS, .D, RHEE, T., Underwater Toolkit: Mixed Reality Object Blending for $360^{\circ}$ Videos. ISMAR 2019 [Demo]

RHEE, T., CHALMERS, A., LOH, I., ALLEN, B., PETIKAM, L., THOMPSON, S., REVILL, T., Mixed reality 360 live: live blending of virtual objects into $360^{\circ}$ streamed video. In ACM SIGGRAPH 2018 Real-Time Live! (p. 5). ACM. [On-stage live demo] 


\section{Contents}

Abstract

Acknowledgements $\quad$ ii

List of Publications $\quad$ v

List of Figures $\quad x i$

1 Introduction 1

1.1 Motivation . . . . . . . . . . . . . . . . . 1

1.2 Research Objectives . . . . . . . . . . . . . . . 4

1.3 Contributions .................. 6

1.4 Thesis Structure . . . . . . . . . . . . . . . 7

2 Related Work 9

2.1 Head Mounted Displays . . . . . . . . . . . . . . . . . 9

2.2 Presence and Social Presence . . . . . . . . . . . . . . 13

2.3 Omni-directional Video . . . . . . . . . . . . . . . . . 14

2.4 Computer Supported Cooperative Work . . . . . . . . . . . . 16

2.5 Networking . . . . . . . . . . . . . . . . . 18

2.6 Collaboration and Communication Systems . . . . . . . . . 22

2.7 Mixed Reality Rendering . . . . . . . . . . . . . . . . . 25

2.8 Summary . . . . . . . . . . . . . . . . . 27

vii 
3 System Implementation $\quad 29$

3.1 Overview . . . . . . . . . . . . . . . . . . . 29

3.2 Design Principles . . . . . . . . . . . . . . . . . 30

3.3 System Setup . . . . . . . . . . . . . . . . . 32

3.4 User Setups . . . . . . . . . . . . . . . . . . 33

3.4 .1 Gear Setup . . . . . . . . . . . . . . 33

$3.4 .2 \quad 360$-Video . . . . . . . . . . . . 34

3.4 .3 Avatars ..................... 35

3.5 Shared Space . . . . . . . . . . . . . . . . . . . . . . 37

3.5 .1 Space Calibration . . . . . . . . . . . 37

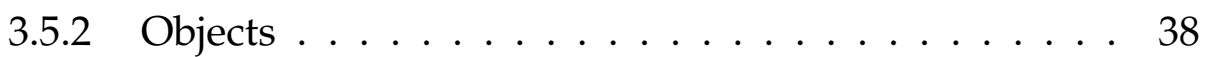

3.5 .3 User Interface . . . . . . . . . . . . . . . . . 38

3.6 Mixed Reality Rendering . . . . . . . . . . . . . . . . . 41

3.6.1 Lighting and Shading . . . . . . . . . . . . . . 41

3.6.2 Shadow Catcher . . . . . . . . . . . . . 42

3.7 Summary . . . . . . . . . . . . . . . . 45

4 Evaluation and Results $\quad 47$

4.1 Setup . . . . . . . . . . . . . . . . 47

4.2 360-Camera Performance. . . . . . . . . . . . . . . . 49

4.2 .1 Results . . . . . . . . . . . . . . . 49

4.3 User Study . . . . . . . . . . . . . . . . . . . . . . 53

4.3 .1 Methodology ................ 53

4.3 .2 Tasks ........................ 55

4.4 Results . . . . . . . . . . . . . . . . 58

4.4 .1 Recorded Data . . . . . . . . . . . 58

4.4 .2 Feedback . . . . . . . . . . . . . 60

4.5 Discussion . . . . . . . . . . . . . . . . 64

4.5 .1 User Presence . . . . . . . . . . . . . . . . . 65

4.5.2 User Satisfaction and Performance . . . . . . . . . . 65

4.5 .3 System Performance . . . . . . . . . . . . . . . 67 
CONTENTS ix

4.5.4 Applications . . . . . . . . . . . . . 68

4.5 .5 Summary .................. 72

5 Conclusion $\quad 73$

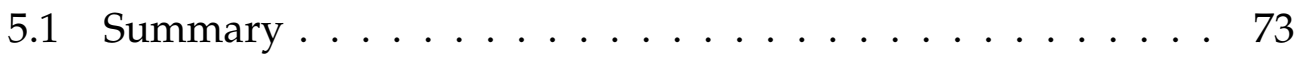

5.2 Limitations . . . . . . . . . . . . . . . . . . 74

5.3 Future Work . . . . . . . . . . . . . . . . . . 75

$\begin{array}{ll}\text { Appendices } & 77\end{array}$

$\begin{array}{ll}\text { A System Instructions } & 79\end{array}$

B Consent Form $\quad 85$

$\begin{array}{lr}\text { C Information Sheet } & 89\end{array}$

D User Evaluation Questionnaire $\quad 95$ 


\section{List of Figures}

1.1 Mixed Reality Spectrum, as suggested by [67]. . . . . . . . 2

1.2 The concept of local and remote users, each in their corresponding spaces. As highlighted by the arrow, the remote user can use MR systems to "teleport" to the local space. . . 3

1.3 The points of view from two users using the RC System to decorate the local space with furniture. The users are using their VR controllers to point at the pot plant, as highlighted through the red and cyan rays. . . . . . . . . . . 5

2.1 From the left; Oculus Rift and HTC Vive VR Headsets. . . . 10

2.2 Pass-through $(\mathrm{a}, \mathrm{b})$ and See-through (c, d) HMDs. . . . . . . 11

2.3 Spatially mapped space using the ZED Camera. . . . . . . . 12

2.4 An example of a 360-image. . . . . . . . . . . . . . 15

2.5 A 360-camera (Samsung 360 Round). . . . . . . . . . . . . . 15

2.6 Image from Microsoft's Holoportation System [75]. The system (Left) and the 3D reconstruction of the user (Right). . . 23

2.7 The SharedSpace MR collaboration system. The image is from [59]. . . . . . . . . . . . . . . . . . . . . 24

2.8 Differential rendering. Images taken from [22]. . . . . . . . 26

2.9 Virtual object composited into a 360-video using real-time differential rendering. Image taken from MR360 [87]. . . . . 27 
3.1 The shared space is where local and remote users can interact face-to-face (shown in purple). The local user uses an AR device to transform the local space into the shared space. The remote user wears a VR HMD to "teleport" to the shared space. . . . . . . . . . . . . . 30

3.2 Overview of the RC System with all components active. . . . 31

3.3 Remote user's avatar as seen from the Local User's perspective. The cyan lines (pointers) represent where the remote user is pointing. . . . . . . . . . . . . 36

3.4 The personal menus for the local user (a), remote user (b), and as an abstract representation when viewed by a different user(c). . . . . . . . . . . . . . . . 39

3.5 A remote user and local user interacting with a shared menu. 40

3.6 Render pipeline for forward differential rendering in Unity. The 360-image is taken from HDRIHaven. com under the CC0 licence. . . . . . . . . . . . . . . . . . 44 41

3.7 A 360-video (a) after it has been projected onto coarse room geometry (b). The yellow dot denotes the location of the 360 -camera and the point from which the 360 -video is projected. . . . . . . . . . . . . . . . . 44

3.8 Rendering virtual objects into the real-world (a) using reconstructed geometry (b), and the final composition as seen by the local (c) and remote (d) users. . . . . . . . . . . . . 44

4.1 The floor plan during the evaluation. . . . . . . . . . . . 48

4.2 The setups for streaming videos captured with the 360 cameras to a different computer (Remote PC) over LAN. . . . . . 50

4.3 Image comparison between the 360-cameras. Please note the better dynamic range and more natural colours seen in (b) and (c), and the stitching artefacts seen in the ghosting on the door (a) and ghosting on the IVR text (b). . . . . . . 52

4.4 Training task. . . . . . . . . . . . . . 55 
4.5 Main task. . . . . . . . . . . . . . . 56

4.6 Shared menu during the training task (a) and main task (b). 57

4.7 Room and shape layout for the second task. Shapes A, B and $C$ were placed by the local user. The remaining shapes were placed by the remote user. Positions 1 and 2 denote where the local user and 360-camera were positioned. . . . 59

4.8 Example applications as suggested by Rhee. et. al. [88]. The left images is the local user's view and the right images is the remote user's view. . . . . . . . . . . . . . . . . . 69

4.9 Two users experiencing a cinematic experience. . . . . . . . 70

4.10 Remote User giving a presentation. . . . . . . . . . . 71 


\section{Chapter 1}

\section{Introduction}

\subsection{Motivation}

Improvements in technology, such as the introduction of phones and video conferencing, has brought about the ability for real-time communication among people who are not situated in the same location. However, this technology is not able to fully recreate the presence and freedom present when people are at the same location.

Within the past decade, head mounted displays (HMD) required for viewing virtual reality (VR) [112, 73] and augmented reality (AR) [5, 57] content have become viable for remote collaboration. Users of VR based collaboration systems are represented as avatars and can interact with other users in a fully virtual environment. Using AR for collaboration allows users wearing AR HMDs and situated within the same physical space to view and interact with virtual contents augmented into the realworld $[58,65]$. Users who are not present in the same physical space as other AR users are often represented as digital avatars. 
VR and AR systems can be considered as a part of a spectrum (Figure 1.1) called the Reality-Virtuality Continuum [67,68]. This spectrum describes the degree of real and virtual contents, with the purely real environment and virtual environment representing opposite ends of the spectrum. Any combination of real and virtual content situated between the two extremes is labelled Mixed Reality (MR).

Figure omitted due to missing permission.

Figure 1.1: Mixed Reality Spectrum, as suggested by [67].

There has been a growing interest in using VR and AR to provide fully virtual or combined virtual and real world spaces for people to interact and communicate. Unlike collaboration done through video conferencing or voice chat, VR and AR gives users an improved sense of realism to perform collaborative tasks by providing experiences that are more similar to face-to-face meetings than what has previously been possible.

Throughout this thesis, local users refer to the users who are physically located within a localised space and remote users are users located in a physically different location. In cases where knowledge of the local space is important for performing a task (for example, remotely interior decorating a space), then collaboration systems using a combination of VR and AR are beneficial. Combining AR and VR into a single system can allow for a space where local AR users can interact and remote VR users can join by "teleporting" to the local space, as shown in Figure 1.2. 


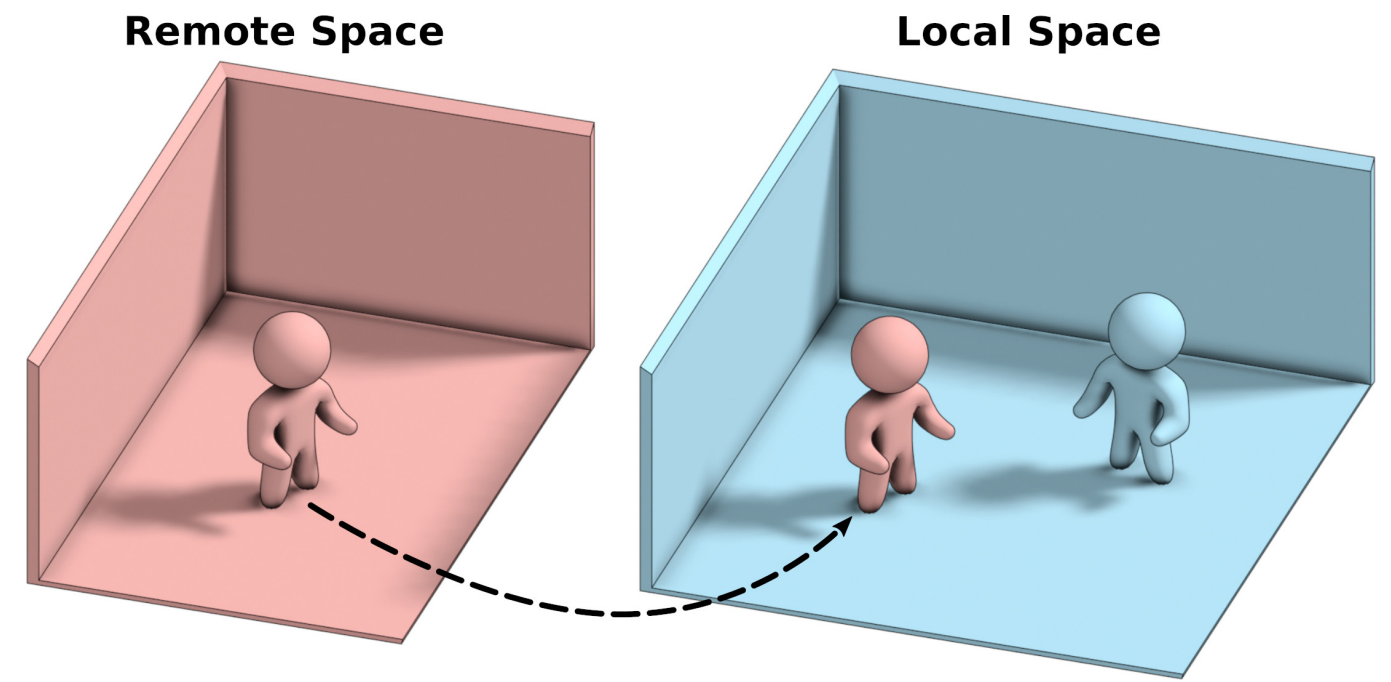

Figure 1.2: The concept of local and remote users, each in their corresponding spaces. As highlighted by the arrow, the remote user can use MR systems to "teleport" to the local space.

When combining real and virtual contents, fidelity should be considered for visual consistency. Fidelity refers to the accuracy to which an effect is reproduced [32]. Throughout this thesis, fidelity refers to the visual consistency between the real-world and virtual objects. Virtual objects that have been composited into the real-world should be rendered with realistic lighting and cast shadows onto the real-world geometry to appear as high fidelity.

MR systems allow AR users to scan and share geometry of the local space with the remote VR users, allowing full freedom to navigate and interact with the space for all users $[65,102]$. However, using a purely virtual environment lacks high fidelity in the visual quality. Scanned geometry has limited texture and model resolution, and requires to be rescanned by local users to capture any changes in the local environment. Communication gestures between users are also partially lost in the avatar representation. 
There has been research into providing remote users with a live-streaming video from a $360^{\circ}$ camera (360-camera) located in the local space. This gives remote users a high-fidelity, live $360^{\circ}$ view of the local space from the position of the camera. Current proposed systems utilising 360-cameras are limited in their functionality. The 360-camera may be coupled with the view of the local user [83], limiting the ability to recreate some communication cues seen in face-to-face communication, such as eye contact. Other 360-camera based systems only focus on verbal and gesture based communication [60, 106] or limit real-world interactions to annotations [59].

\subsection{Research Objectives}

This thesis proposes a new system for providing collaboration between local and remote users within an MR environment. The goal of this system is to simulate face-to-face interaction for users who are not co-located in the same space. A live streaming 360-camera set up in the local space provides remote users with a real-time omni-directional view of the local space that can be viewed through a VR HMD. The local user is able to use an AR HMD to see the remote user represented as an avatar. The 360-camera is further utilised to provide real-time believeable lighting and composition of digital objects into the real world [87].

A working prototype system has been built and evaluated with a technical performance analysis and user study. The technical performance tests the suitability of the system for real-time interaction, communication and collaboration. A user study with users performing collaborative tasks was also conducted to gain feedback from participants regarding user experience and satisfaction of the system. The evaluation found users were satisfied with the collaboration and communication they experienced.

Throughout this thesis, the working prototype will be referred to as the Remote Collaboration System (RC System). An example the RC System can be viewed in Figure 1.3. 


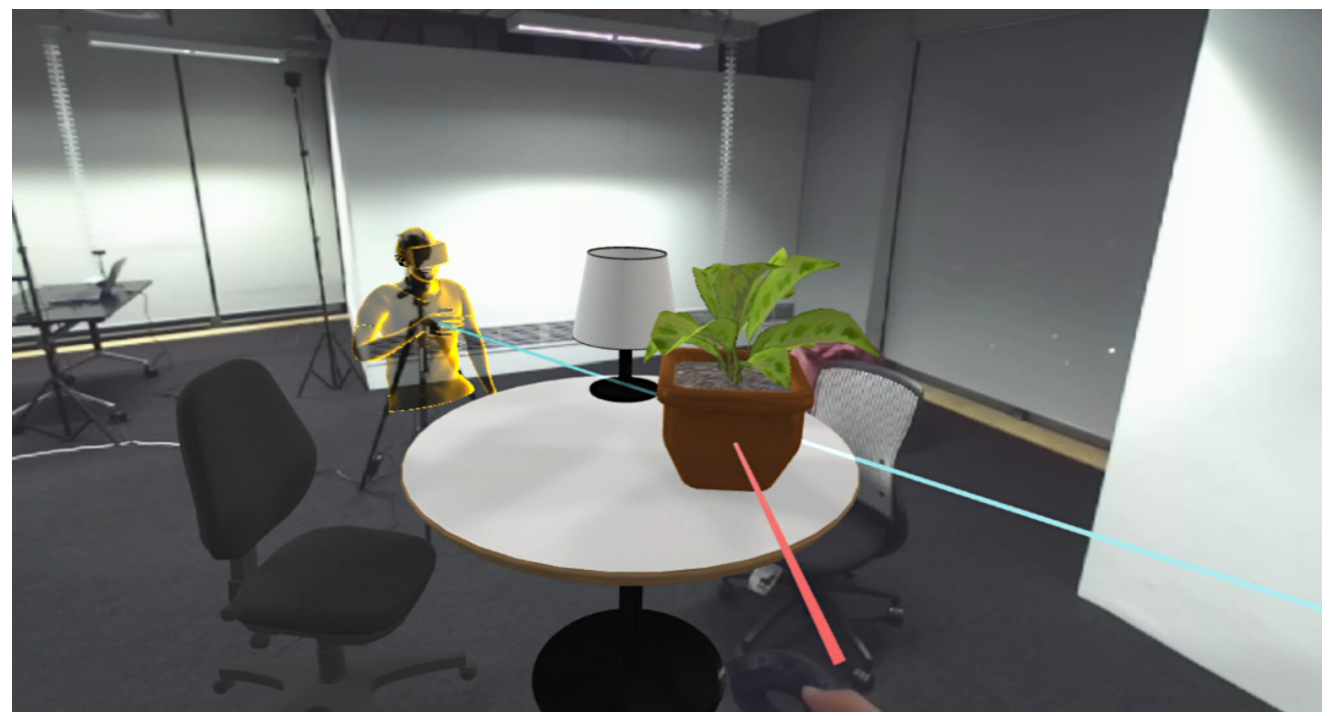

(a) Local View

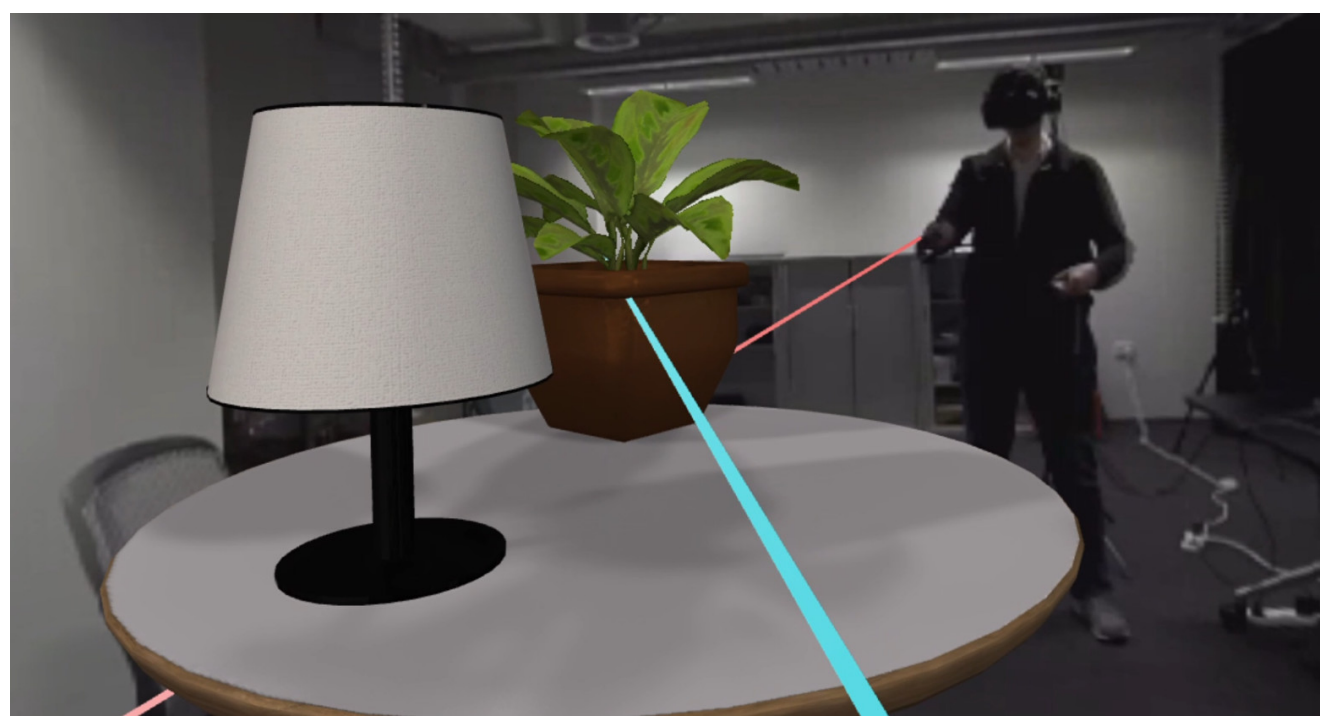

(b) Remote View

Figure 1.3: The points of view from two users using the RC System to decorate the local space with furniture. The users are using their VR controllers to point at the pot plant, as highlighted through the red and cyan rays. 


\subsection{Contributions}

The main contributions of this thesis is summarised as follows:

- A system is developed and integrated into a commercial game engine to enable local and remote users to communicate and collaborate in MR.

- Evaluated three different 360-cameras for quality and latency, highlighting the benefits for each camera and concluding which works best for a real-time setup.

- Conducted an evaluation of the performance of the system and user satisfaction when using the system.

- Discuss possible applications [88] where this research can be utilised and introduce some new application ideas.

- Adapted lighting technology based on MR360 [87] for AR devices and collaborative setups. 


\subsection{Thesis Structure}

This thesis is structured as follows:

Chapter 2 Summarizes related work in collaboration and communication based systems using VR, AR or both. Mixed reality rendering will also be discussed briefly.

Chapter 3 Provides an overview of the system setup and explains the different components, software and hardware that make up the system, along with the reasons behind the design choices.

Chapter 4 Evaluates the research based on the technical performance and user experience. The testing setups, methodologies and analysis of the results for both evaluations will be discussed. Possible applications where the system is beneficial are also discussed.

Chapter 5 Summarizes and concludes the thesis, presents limitations of the system and discusses future work. 


\section{Chapter 2}

\section{Related Work}

\subsection{Head Mounted Displays}

There has been interest in recreating the experience of being in remote or digital places for many years. As early as 1832, Sir Charles Wheatstone [115] found that the sense of 3D depth experienced in the real-world can be simulated in traditional, monoscopic 2D images. Viewing stereoscopic images - two 2D images taken at the same time with a slight horizontal offset - such that the left and right eye can see the corresponding images, simulates the natural distance between the eyes giving the illusion of 3D depth.

A proposed design [39] for the first display based head-mounted display (HMD), the Telesphere Mask, was patented in 1960, the design of which is very similar to modern HMDs. A couple of years later, the Headsight [21] was developed to allow people to view a live-streaming, remotely controlled camera through a HMD. The camera could be controlled by rotating the HMD. Ivan Sutherland proposed an idea to create virtual worlds to experience [103], an idea he completed 3 years later [104]. This device was the first AR headset invented, able to render 3D geometry wireframes at a frame-rate of $30 \mathrm{~Hz}$. The tracking device used for this headset was called the Sword of Damocles. 
Since then, technology has continued to improve providing better visual quality and user experience. Early research in VR and AR focused on solving the hardware limitations of the medium. During the 90s, interest in VR increased with some companies, including SEGA and Nintendo, developing consumer-based HMDs. However, these projects were met with mixed success because of limitations in hardware at the time. In 2011, a prototype was created for a new headset called the Oculus [73]. In 2016, Oculus and rival company HTC, maker of the Vive [112], released consumer VR HMDs (Figure 2.1) to the market. With recent technical advances and increased availability of HMDs, research has shifted to exploring use cases and experiences [26].

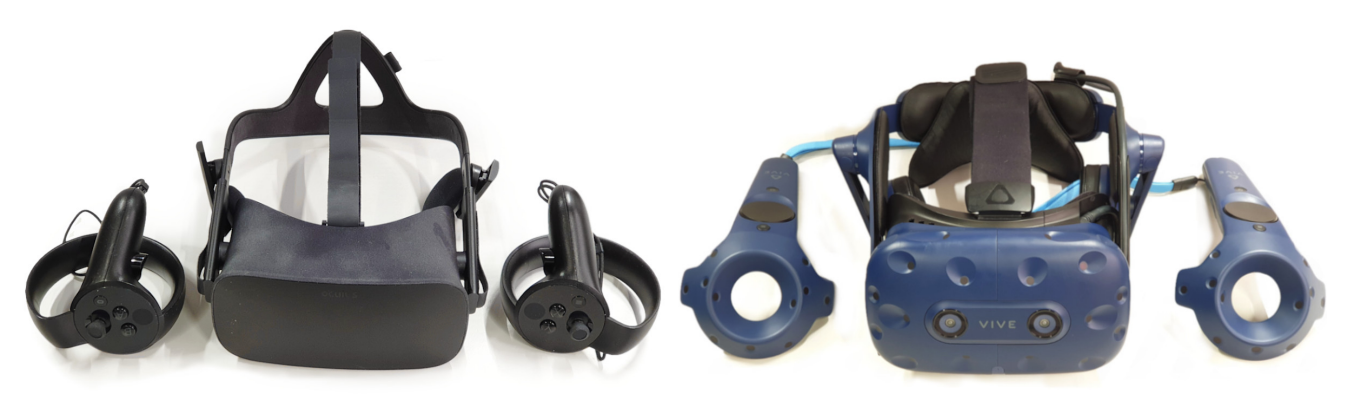

Figure 2.1: From the left; Oculus Rift and HTC Vive VR Headsets.

Current consumer based VR HMDs are designed to display content at $60-144 \mathrm{~Hz}$, with higher refresh rates providing smoother experiences. Released in 2016, the resolution of the Oculus Rift and HTC Vive is 1080x1200 pixels per eye. More recent HMDs can display content at even higher resolutions, even as high as 3840x2160 pixels per eye [1]. Using higher screen resolutions reduces the screen door effect, an immersion damaging effect created when users can identify the individual pixels. 


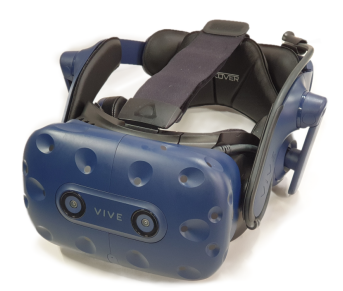

(a) HTC Vive Pro

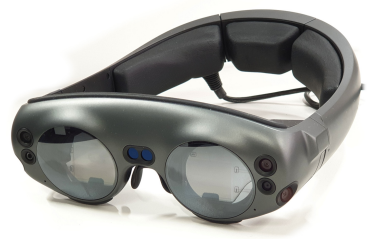

(c) Magic Leap

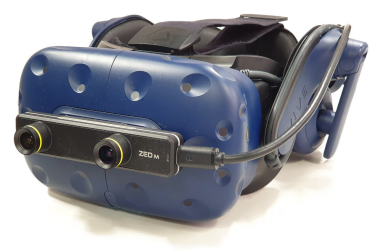

(b) ZED Mini camera attached to an HTC Vive

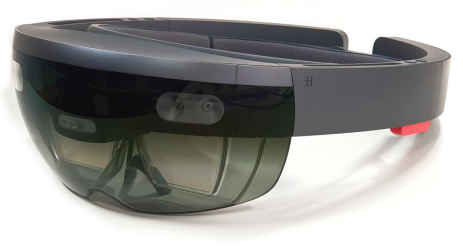

(d) HoloLens

Figure 2.2: Pass-through (a, b) and See-through (c, d) HMDs.

VR HMDs can be expanded to support MR by displaying stereo footage streaming from a camera attached to the front of the HMD. This type of HMD is called a pass-through display (Figure 2.2) and allows virtual content to be rendered on top of the footage. There are limitations with camera resolution and latency, which can be mitigated by using either a low resolution 640x480 video stream or a high resolution 4096x2048 video stream but at a low frame-rate [3]. Alternatively, specialised AR headsets using see-through displays (Figure 2.2) can be used to overlay virtual content directly on top of the real-world. These types of displays work by rendering the virtual objects using light. The limitation to this approach is that darker colors will appear transparent rather than opaque, with black rendered fully transparent. Shadows and similar effects need to use alternate techniques, such as negative shadows [66], to appear visible. Current wireless, see-through AR HMD are also limited in the field of view, only able to render roughly 45 degrees compared to 110 degrees common in VR HMDs. A wider field of view similar to VR HMDs can be achieved 
when the AR HMD is connected to a computer, such as with the Meta or DreamVision HMDs.

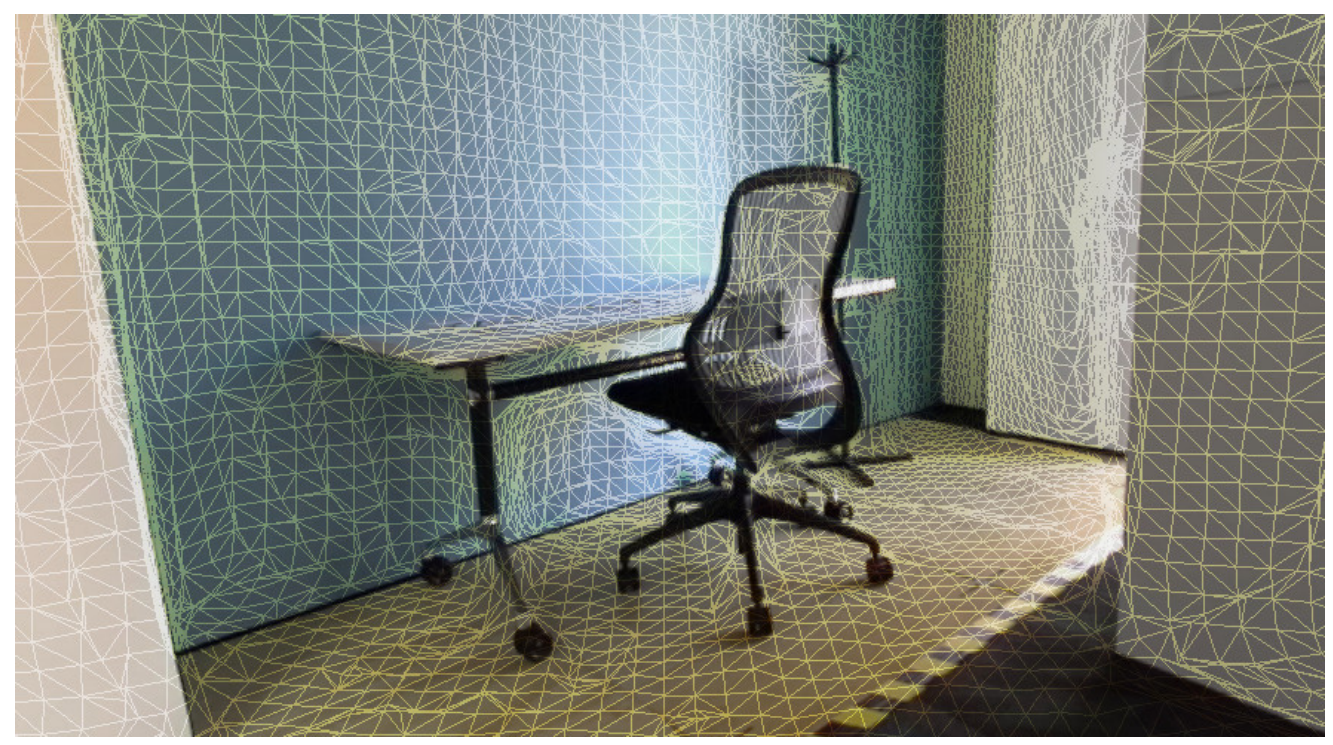

Figure 2.3: Spatially mapped space using the ZED Camera.

AR HMDs often have the ability to estimate depth in front of the user and reconstruct 3D geometry (also called spatial mapping, Figure 2.3) of the surrounding area. Depth estimation enables the occlusion of virtual objects behind physical objects and geometry reconstruction provides a coarse mesh for physics simulation when virtual objects collide with realworld objects. AR HMDs will perform inside-out tracking by using cameras built into the HMD to track points in the real-world, which is used for calculating the location and orientation. This tracking allows users to experience the virtual world with 6 degrees of freedom (DoF). That is, being able to view the virtual content with any view orientation and from any position. However, current inside-out tracking is not always stable, and the positions and orientations of virtual objects can drift over time. VR HMDs traditionally use external tracking devices to achieve better tracking, as long as there is a non-obscured line of sight between the tracking device and HMD. 


\subsection{Presence and Social Presence}

The experience of viewing virtual reality content is strongly connected to the idea of tele-presence [101]. Presence has been defined using multiple definitions: feeling of being physically present with the virtual objects [94], or as a combination of spatial presence, involvement and realism [93]. Ultimately, the definitions can be summarised as a complete feeling of "being there" [37] in the virtual environment.

Many factors can support or hinder one's feeling of presence. HMDs containing positional and rotational freedom provides a heightened sense of presence compared to traditional displays [17]. Preliminary results by Bouchard. et. al. [13] suggest that people who perceive the virtual environment as a real place had a significant increase in feeling presence. Factors such as mismatching visual and sensory information, when felt by the user, can negatively affect the feeling of presence [114]. Regenbrecht et al. [86] were able to maintain a high sense of presence by decreasing the visual quality of the real world environment to match the style of the virtual objects. Doing so provided visual coherence in rendering quality between the real and virtual world. However, it has been shown that increasing the realism of the objects and environments can also increase the sense of presence $[44,78]$. High resolution textures and meshes, alongside high quality lighting and shading, add to the sense of realism and presence a user may experience.

Similar to presence is the idea of social presence, the sense that someone is present with other people in a remote or shared environment [117, 16, 33]. People who are not co-located in the MR space need to be represented as an avatar, and how the person appears has an impact on not just social-presence, but presence as well [33]. Realistic human avatar have been shown to provide better presence than abstract avatars [38]. A recent study has found that having avatars with higher realism brings an improved sense of presence [45]. Given three avatar possibilities (head and 
hands only, top half of body and full body) combined with either realistic or cartoon style rendering, the high realism, full body avatar provides the highest sense of presence. The worst sense of presence comes from avatars displaying only a head with hands. Another study found the difference in social-presence between using partial body and full body was not significantly worse, but using motion generated from the user as opposed to predefined animation was better [56].

One concern is that the user does not experience the uncanny valley, a term used to describe when objects, particularly humans, appear not quite realistic and leave viewers feeling revulsed [69]. This phenomenon has been found to damage the viewer's sense of presence in a virtual world [14]. In VR, the uncanny valley can arise from realistic user interactions [64] and from interactions where objects the user touches does not match the expected virtual appearance [24]. Humanoid avatars that fail to move human-like can also fall into the uncanny valley [100].

\subsection{Omni-directional Video}

While traditional VR experiences use a fully virtual environment, another option is the use of omni-directional images [18] (Figure 2.4) and videos [82] (360-video). A 360-video provides viewers the freedom to view the footage in any direction. The footage can be projected onto a sphere with a virtual camera at the center to allow users to view the footage. Many video hosting and social media sites, including YouTube [34] and Facebook [27], now include the ability to play 360-video. Because 360-videos cover an entire sphere and is commonly viewed in HMDs, it should have a higher resolution than traditional 2D video (1920x1080 pixels) to reduce blurriness. It is common for 360-videos to have a resolution of 4096x2048 pixels. However, using a resolution as high as $8192 \times 4096$ pixels is possible but requires high-end hardware. 


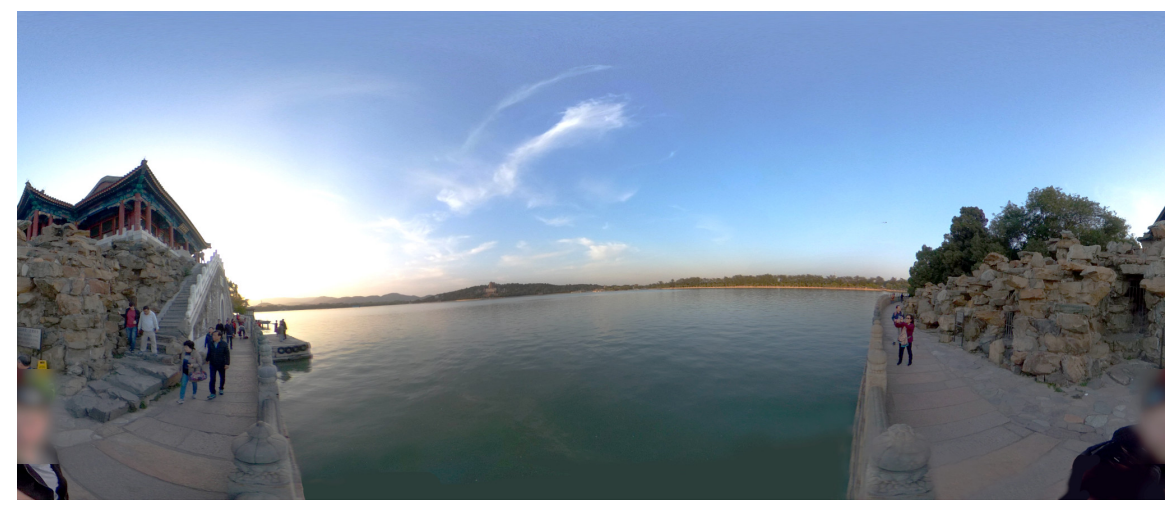

Figure 2.4: An example of a 360-image.

Traditional 360-video is monoscopic, lacking any depth to the viewer. It is possible to use two different panoramic videos generated from two sets of camera positions to reproduce the stereo effect $[42,81]$. Typically, the left eye will be stored in the top half of the video and the right eye in the bottom half, either halving or doubling the vertical resolution. Because the stereo-view is pre-baked, tilting the user's view or looking directly up or down will break the 3D illusion.

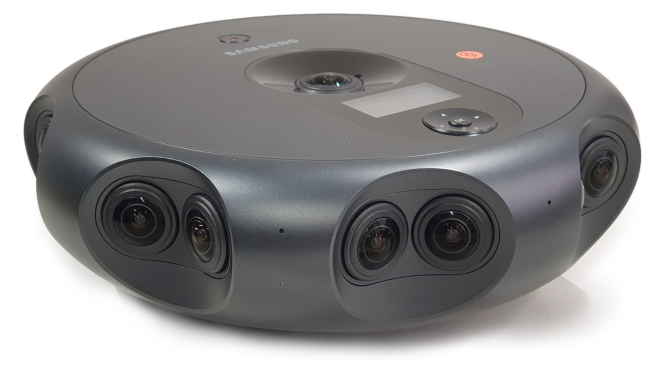

Figure 2.5: A 360-camera (Samsung 360 Round).

Cameras designed for recording 360-videos use multiple lenses to capture the full sphere surrounding the camera. These cameras are becoming more readily available, ranging from affordable consumer based cameras under \$1000 (e.g. Theta Ricoh, Samsung Gear 360) to professional cameras, including the Samsung 360 Round (Figure 2.5). Computer software 
or drivers will take the footage from each lens and stitch it into a single 360-video, and, in some cases, is able to stitch and broadcast the footage in real-time. Because of the higher resolution requirements, 360-video requires a faster network connection than standard $2 \mathrm{D}$ videos in order to playback in real-time. However, it is possible to reduce the amount of data streamed by only streaming the small portion of the 360 -video visible to the user $[41,98,71]$.

The use of 360-videos has been shown to provide a high sense of presence, leading the users to feel more emotionally attached to the experience $[28,29]$. Using higher resolutions and better quality encoding, especially when viewed through a VR HMD, helps to add a better sense of presence [109]. In the same study it was found that having little to no motion was best for reducing nausea, however having a small amount of motion improves presence. Evidence suggests that viewing 360-content in a HMD provides better spatial awareness and enjoyment over traditional display systems [63]. Viewing stereoscopic 360-video has not been found to improve presence over monoscopic 360-video [11]. The RC System incorporates 360-video into a collaborative system because of the high sense of presence 360-videos provide.

\subsection{Computer Supported Cooperative Work}

Computer Supported Cooperative Work (CSCW) is the field of research interested in exploring the theory of communicating and collaborating using software and hardware, along with solving issues that arise. Strongly related to CSCW is groupware, the software and hardware that enables people to communicate and collaborate. CSCW tries to identify how people use groupware to reach a common goal and what aspects of the groupware improves the journey to their goal. Interactions with groupware may happen at different times and places. The different conditions can be described using a matrix [48] (Figure 2.1), separating the interaction as taking 
place at the same time (synchronous) or at different times (asynchronous). Likewise, the collaborators may be situated in the same space (co-located) or be in completely different places (remote). Examples of each type of interaction includes face-to-face meetings (synchronous, co-located), videoconferencing (synchronous, remote), post-it notes (asynchronous, co-located) and email (asynchronous, remote).

\begin{tabular}{|c|c|c|}
\hline & $\begin{array}{c}\text { Synchronous } \\
\text { (Same Time) }\end{array}$ & $\begin{array}{c}\text { Asynchronous } \\
\text { (Different Time) }\end{array}$ \\
\hline $\begin{array}{c}\text { Co-located } \\
\text { (Same Place) }\end{array}$ & $\begin{array}{c}\text { Face to Face } \\
\text { Interaction }\end{array}$ & Continuous Task \\
\hline $\begin{array}{c}\text { Remote } \\
\text { (Different Place) }\end{array}$ & Remote Interaction & $\begin{array}{c}\text { Communication } \\
\text { \& Coordination }\end{array}$ \\
\hline
\end{tabular}

Table 2.1: Time-Space Matrix [48]

CSCW has identified design principles for improving user experience, communication and collaboration when using groupware. One example is the importance of maintaining awareness among the users [23, 92, 19]. Cues, including eye-gaze [76, 40], hand gestures [90, 43, 116, 89] and annotations [43], improves communication and collaboration between local and remote users.

When groupware is used in a synchronous, remote interaction, there arises the issue of latency. Groupware based on real-time communication, including voice chat and video conferencing, must capture the data from a user and send that data over a network. Upon receiving the data, the groupware for the other user must take the data and decipher it in order to express the data in a comprehensible format. Latency is the time between when the data is captured on one end and outputted on the other end. Ideally, latency should be as low as possible, because even latency as small as $200 \mathrm{~ms}$ can negatively affect collaboration [79]. The latency of content shown in VR HMDs should also be kept below 15ms to reduce user discomfort or motion sickness [25]. The refresh rate of content in VR HMD 
is normally $11 \mathrm{~ms}$. However, high visual quality can negatively affect the performance of VR systems, leading to a decrease in the refresh rate and causing discomfort. Groupware built for remote interaction also requires networking to enable the interaction. The networking should maintain low latency for smooth communication and collaboration between users.

\subsection{Networking}

A computer network is a connected group of computers that can share information among other computers in the group. Communication is performed using network protocols. The most basic network protocol is the internet protocol (IP), which sends packets split into two parts, the header and data. The header is a small piece of information used to ensure the packet arrives at the correct destination. The number of packets sent over a network uses bandwidth, which is the measure of the amount of data being transferred over a network.

Two commonly used protocols for networking are the Transmission Control Protocol (TCP) and the User Datagram Protocol (UDP), both of which are built on top of the IP. TCP is a reliable connection oriented protocol that ensures data will be received in the same order sent. A connection between two computers need to be established before sending data and received data needs to be acknowledged. Although TCP ensures that data will arrive correctly, this reliable connection comes at a cost of added latency and bandwidth. Unlike TCP, UDP is a connection-less protocol, and has no guarantee that data sent over a network will arrive at the destination. This unreliability of packet delivery requires minimal bandwidth and can be beneficial in cases where data is constantly changing and lost packets quickly become out of date. Reliable packet delivery can be simulated on UDP if needed, but at the cost of additional bandwidth. 
Both protocols are used for different purposes. UDP is often used for real-time applications where delivery speed is more important than reliability (e.g. voice chat). TCP is used when reliable, ordered delivery of packets is important (e.g. file downloading). There are also protocols built on top of TCP and UDP. A common protocol for videos streaming is the real-time multimedia protocol (RTMP). This protocol is built on top of TCP and is commonly supported in video broadcasting and live-streaming.

Architectures for managing network systems include the client-server network and the peer-to-peer (P2P) network. With client-server, there is a central computer (server) acting as the source of information. Clients do not directly interact with each other but can send information to and request information from the server. P2P has no central source of information but rather information is distributed equally among users. Some advantages for each architecture are listed as follows:

\section{Client-server}

- The server provides a centralised point containing the true state of the system. Clients can request information when needed, unlike $\mathrm{P} 2 \mathrm{P}$ where synchronisation can become challenging since no peer is authoritative of information.

\section{P2P}

- In a client-server architecture the server can require large amounts of bandwidth for sending and receiving data to and from clients. In the P2P architecture, bandwidth is distributed among all of the peers.

- A P2P network does not rely on a server to remain connected. 
The RC System implemented in this thesis uses a client-server setup and UDP for networking. Only two computers are networked in the system. The client-server architecture has been chosen over the P2P architecture because it is faster and easier to implement. UDP has been chosen because the RC System requires frequent updates that quickly become obsolete. Reliable packet delivery on UDP is simulated when needed.

\section{Networking Optimisations}

Sending large amounts of raw data over a network wastes resources, leading to problems including packet loss and unstable connections. Optimisation techniques have been created as a way of reducing bandwidth while also enabling larger amounts of data to be sent. Data can be compressed using either lossless or lossy compression. Lossless compression compresses data without losing any information. On the other hand, data can be compressed at a larger ratio using lossy compression, but at the cost of quality or precision.

A simple method of data compression is bit packing. Numbers are stored on computers using the binary system, a sequence of 1's and 0's (bits) used to represent numbers. For example, a standard integer is stored using 32 bits. If four integers need to be transported over a network but will always fall within a value range of 0 and 255, requiring only 8 bits to store, then the four integers can be packed into a single 32 bit integer. The first 8 bits will represent the first integer, second 8 bits the second integer and so forth. In this example the data has been compressed to a quarter of the original size without losing any information.

Decimal numbers can be compressed using a similar technique, however, at the loss of some precision. For example, a decimal number can be reduced to a quarter of the size by remapping the value to the range of a byte (0 to 255). This is an example of lossy compression. If higher precision is required than more bits will need to be used. This compression method can be optimised depending on the use-case. For the case of stor- 
ing a position (96 bits), the vertical y axis may not require the same range as the horizontal $x$ and $z$ axes. This information can be packed into 32 bits using 11 bits for the $x$ and $z$ axes and 10 bits for the $y$ axis, providing better precision and range for the axes that require it.

Orientation of objects is often described using quaternions. Quaternions are made from four decimal numbers, requiring a total of 128 bits to store. One method of compression is to convert quaternions to Euler angles. However, this method still requires three decimal numbers (96 bits) to represent the angles. The quaternion can be bit-packed into a single integer (32 bits) at the cost of lost precision. An improved variation of this method that is able to gain higher precision is the smallest three method [2].

This method works by packing the three smallest numbers in a quaternion into a 32 bit integer. The fourth number can be calculated from the three numbers using the equation $1=\sqrt{x^{2}+y^{2}+z^{2}+w^{2}}$ to solve for the missing number. The first two bits are used for identifying which of the four components is the largest number. If the largest number is negative then the smallest three numbers are negated. The remaining thirty bits are broken down to 10bits for each of the three remaining components. Precision can be improved by scaling the three numbers to the range between $-\frac{1}{\sqrt{2}}$ to $\frac{1}{\sqrt{2}}$.

Bit packing is a simple solution, but better compression ratios can be achieved using more complicated solutions. Sending multimedia over a network is highly bandwidth intensive. For video, the need to encode data is even more important, with a typical uncompressed 1920x1080 resolution video requiring $149.3 \mathrm{mb}$ per second! A simple approach is to compress each individual frame, such is the case for Motion JPEG (MJPEG) format that uses the JPEG image format for each frame. Better compression can be achieved while gaining higher quality by analysing the video frames to better encode frames or predict future frames. One of the standardised video encoding algorithms using these techniques is H.264 en- 
coding. This algorithm is able to compress a 1920x1080 resolution video to use only $1 \mathrm{mb}$ per second while maintaining an acceptable quality.

These optimisations have been used in the RC System to improve networking performance of the system. High resolution video is streamed using the H.264 encoding, and low resolution video is streamed using MJPEG encoding. Bit packing is used for non-video related networking within the system.

\subsection{Collaboration and Communication Systems}

The increase of VR and AR technology has brought new areas of CSCW research. Remote collaboration has been done for several years through mediums such as video conferencing. Now that VR has become more widespread, a new area of collaboration exists where users can now join in a virtual space, which can be beneficial if information of the real-world local space or remote space is not required. AltspaceVR [46] and Mozilla hubs [4] are a couple of examples of these types of systems. AR systems have also shown potential for collaboration through shared spaces and virtual object composition [62, 10].

Research has started exploring using a combination of AR and VR. 3D reconstructed geometry of the local space can be sent to the remote user, to provide them with the freedom to explore the local environment with full 6 DoF $[102,8]$. Virtual representations of objects in the physical space can also help with collaboration [74]. Microsoft Research created a system capable of live-streaming 3D reconstructions of people in a remote space to a local user wearing a HoloLens [75](Figure 2.6). Although well received, there were some negative responses from the user evaluation highlighting visual artefacts in the reconstruction. Their system also requires multiple high-end hardware to process and stream the reconstruction in real-time.

There are also solutions that do not require any of the users to wear HMDs. For instance, using projectors to project the avatar of the remote 
Figure omitted due to

missing permission.

Figure 2.6: Image from Microsoft's Holoportation System [75]. The system (Left) and the 3D reconstruction of the user (Right).

user onto a wall can provide local users with a sense of social-presence [20, $80,91]$. However, this method is limited because the avatar must be projected onto a surface as a $2 \mathrm{D}$ projection.

Wearable cameras live-streaming to remote users can be used to aid communication [51, 12]. Velamkayala et. al. [110] found that video conferencing using the HoloLens improved the collaboration in terms of errors made and time taken compared to using mobile devices. Live streaming video of the local space, along with corresponding depth, can be sent to the remote user $[95,30,31,9]$. A potential limitation of wearable cameras is that the view of the remote users can become fully or partially dependent on the local user. View independence between the local and remote user improves the speed of collaboration [105] and provides freedom for the remote user to view the space unaffected by other users [54].

A project called the Beaming Project proposes using a VR HMD along with haptic feedback devices for the remote user to feel present in the space. The local user can see an avatar projected onto a wall or sphere to represent the remote user [99]. Further work on the Beaming Project explored using robotic humanoids to represent users, allowing transported users to interact with the physical environment [70,55]. Users control and see the world through the robot. The main limitation to this work is the cost of the setup and the lack of formal evaluation of the complete system. 
Recent research has explored using live-streaming 360-cameras to bring remote users into the local space. Remote users are able to view the local space through a HMD or mobile smartphone device [106] or even by navigating a web browser based interface viewing and interacting with the 360-video [97]. Lee e. al. [60] proposed a system where the remote user could view the local scene through a 360 live-stream video. A device was placed next to the remote user to provide $4 \mathrm{D}$ effects (e.g. wind or heat) for better immersion.

Figure omitted due to missing permission.

Figure 2.7: The SharedSpace MR collaboration system. The image is from [59].

The JackIn head system [52] live-streamed a 360-camera device worn by the local user to the remote user, enabling simple communication and collaboration. A different system utilising a wearable 360-camera [59] (Figure 2.7) was proposed for collaboration where the local user could wear a see through AR display along with a $360^{\circ}$ live-streaming camera. The remote user could view the live-streaming 360-video and annotate parts of the video. Interaction with the scene was limited to overlaid information and the remote user's view was partially coupled to the local user. An- 
other prototype tested different avatar visualisations and the best placement of the 360-camera in a multi-scale setup [84]. A study [108] was conducted exploring collaboration using a 360-video compared to 3D reconstructed geometry. It concluded that 360 -video provides better collaboration and presence compared to 3D geometry.

\subsection{Mixed Reality Rendering}

A long running topic in computer graphics is the challenge of rendering virtual objects into a real-world photo or video, such that the virtual object appears indistinguishably realistic compared to the rest of the image. As discussed in Section 2.2, matching the visual quality between the virtual objects and the real-world helps to improve the sense of presence experienced by the users.

One of the best methods for realistic rendering is path tracing [49]. Traditionally, this method is only suitable for offline rendering. However, starting in September 2018, Nvidia has released graphics cards that can perform path-tracing utilizing Microsoft's DirectX Raytracing (DXR). Support for RTX grew in 2019, with the Unity Engine, the program used during development of the RC System, introducing support earlier that year. Unfortunately, because the support was still experimental until later in 2019, the implementation of this thesis makes use of existing, standard GPU based rendering research.

Other common solutions to rendering real-time diffuse lighting is with spherical harmonics [85] or image based lighting [22]. Debevec et. al. [22] presented differential rendering for achieving physically accurate composition results (Figure 2.8). Geometry representing real-world geometry is rendered with and without virtual objects to capture shadows and other lighting effects. The difference between the two images (Figure 2.8(c)) is subtracted from the original image to create realistic lighting composition. Real-time ray tracing [50] has also been used to create realistic composi- 
tions. A virtual geometry representation of the real word scene is required for accurate shadowing between the virtual and the real world. Geometry can be reconstructed using RGB-D cameras (e.g. image depth[35, 61], spatial mapping[15]), camera motion $[107,77]$ or LIDAR. Plane detection is a simplified but common solution for augmented reality [53], and is used in ARCore [6] and ARKit [7].

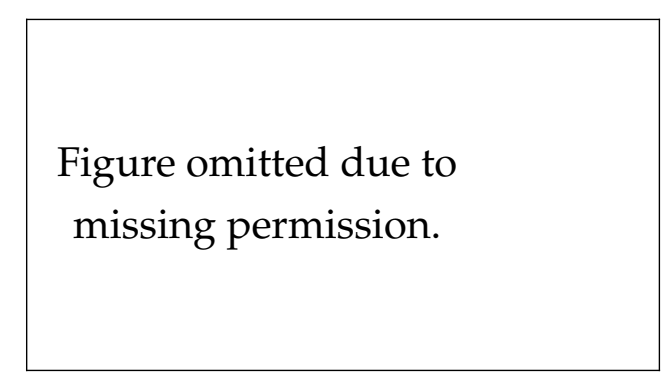

(a) Original Image

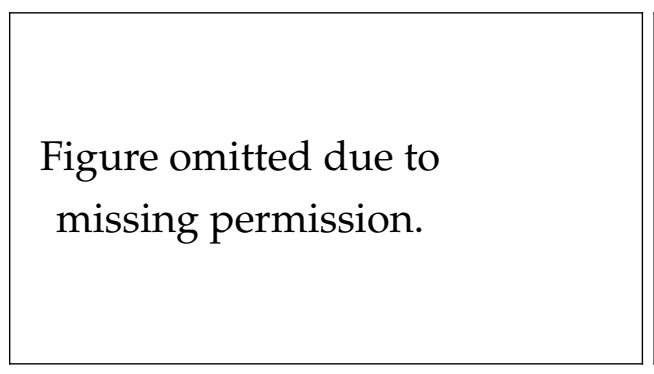

(c) Shadows on Real-world
Figure omitted due to missing permission.

Figure omitted due to missing permission.

Figure 2.8: Differential rendering. Images taken from [22].

Image based lighting uses a high dynamic range 360-image of the scene as a light source [22]. Most 360-videos and cameras do not capture the full range of light required for HDR rendering. Iorns et al [47] uses inverse tone-mapping to mitigate this problem. A moving 360-video can be used to provide spatially varying lighting and reflections [107]. Rhee et al [87] combined 360-videos, image based lighting, light detection and differential rendering to achieve real-time composition of virtual objects into 360-videos, suitable for viewing with head mounted displays (Figure 2.9). 


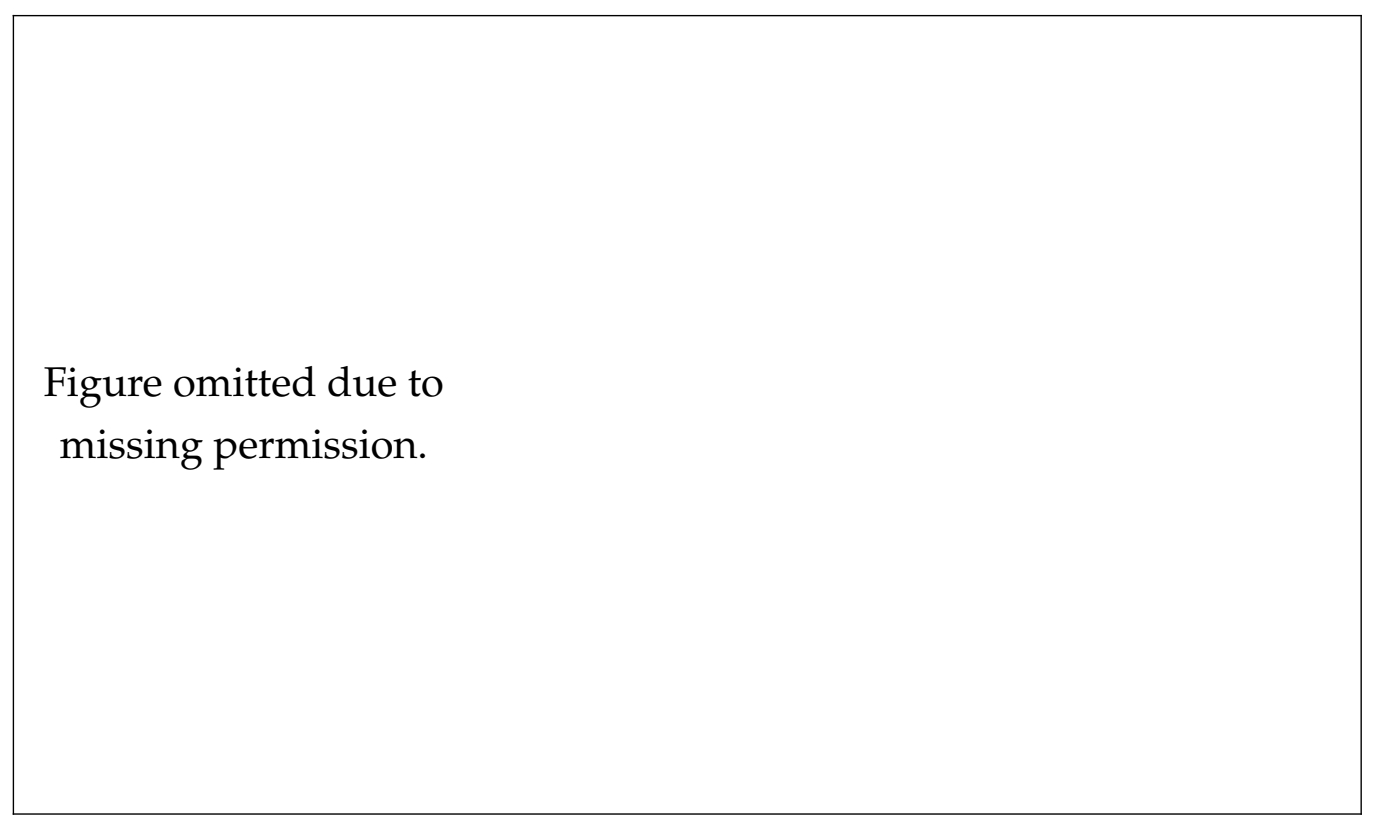

(a) Final Composited Image

Figure 2.9: Virtual object composited into a 360-video using real-time differential rendering. Image taken from MR360 [87].

\subsection{Summary}

This thesis presents the RC System, a new MR collaboration system that incorporates remote communication and collaboration with high fidelity rendering and composition of virtual objects into the real-world. For remote users, virtual objects are composited into a live-streamed 360-video that can be viewed by the remote user through a VR HMD. Local users can see those objects and an avatar representing the remote user composited into the real-world using an AR HMD.

Previous research has explored collaboration using live-streamed 360videos to present a view of the local space to remote users. However, many of these proposed systems focus on shared view collaboration between local and remote users. Other systems that provide standard faceto-face communication often do not provide a high fidelity view of the 
local space to the remote users or have limited virtual augmentation of the local space. Many of these systems are designed around training and assisting use cases, such as remotely guiding a person to perform a task.

The RC System differs from previous work due to a combination of several factors. The system's design focuses equally on the local and remote users and on the high fidelity visuals that aim for realism comparable to the 360-video and the real-world. Another goal of this system is to recreate the feeling of face-to-face communication, so that all users feel as though they are co-present in the same location despite being in different locations physically. The setup of the system is also cost effective when compared to similar systems, such as Holoportation [75] or the Beaming Project [99], only requiring HMDs and VR-ready PCs for each user and a 360-camera. The RC System can be adapted for many applications, not just remote training and assisting. Many different use cases are discussed in section 4.5.4. These differences create a system that provides an interactive, multi-user experience to foster remote communication and collaboration for many situations. 


\section{Chapter 3}

\section{System Implementation}

\subsection{Overview}

The RC System is about recreating the feeling of face-to-face communication and collaboration for people who are not physically located in the same space. A person situated in a remote location can use the system to feel as if they have been physically transported to a new location. Likewise, people within that new location will feel as though the remote person is physically in the same space with them. The system simulates this feeling using an asymmetric mixed reality setup.

Users of the system can belong to either the shared space, local space or remote space (Figure 3.1). The shared space is where all users are able to see and interact with each other and the virtual objects. Users physically situated in the local space share the space with a live-streaming 360camera. They can transform the local space into the shared space by wearing an AR HMD. Local users will need to calibrate their setup in order for object and user positions to align correctly with what other users see. The remote users can put on a VR HMD to enter the shared space, viewing the space from the perspective of the 360-camera. 


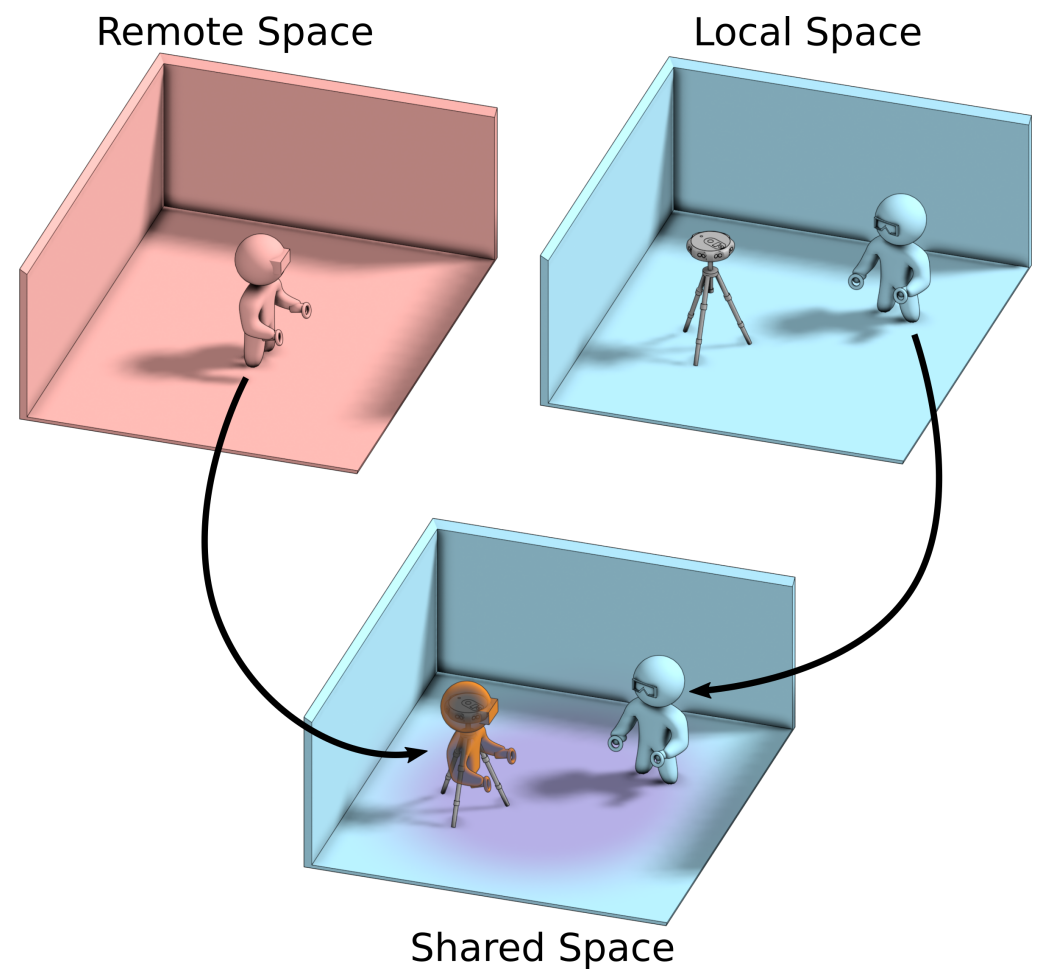

Figure 3.1: The shared space is where local and remote users can interact face-to-face (shown in purple). The local user uses an AR device to transform the local space into the shared space. The remote user wears a VR HMD to "teleport" to the shared space.

\subsection{Design Principles}

The RC System was designed to reflect some of the principles described through CSCW to ensure a platform where collaboration would be easily accomplished. The core principles can be described as follows:

\section{- Awareness}

- Users should be able to:

* see where each other is looking.

* use basic cues (i.e. pointing) to aid communication. 
- Users should:

* be aware of interactions performed by other users.

* have spatial awareness of the shared space.

\section{- Precision}

- The controls should be precise to allow for smooth interaction.

- Communication should be precise and clear.

\section{- Presence}

- Users should have a sense of:

* social-presence with each other.

* being in the same physical space.

- Virtual objects should appear as a part of the shared space.

Many design choices were made in the system to address these principles. A diagram showcasing the system with all components active can be seen in Figure 3.2.

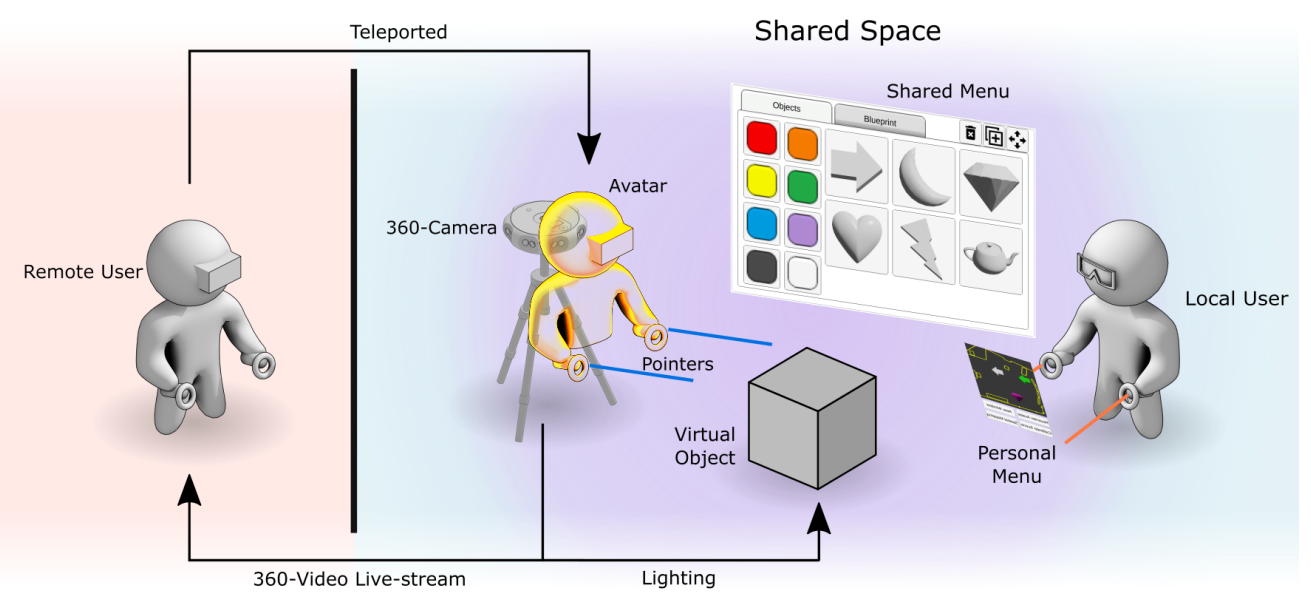

Figure 3.2: Overview of the RC System with all components active. 
The use of real-time streamed 360-video to represent the remote user's view provides a spatial awareness of the shared space and tele-presence for the remote user. Likewise, the remote user being aware of the shared space helps to give the illusion to the local user that they are sharing the space. The local user views the remote user as an avatar, helping to give them awareness of what the remote user is doing. Furthermore, the 360video is used to provide believable lighting to help ground virtual objects in the scene, giving better co-presence between virtual objects and the environment.

Information is visible to local and remote users as frequently as possible. Virtual objects are visible and can be interacted by all users. Interfaces have been split into two systems. Shared menus are global, owner-less windows that are visible and interactable by all users. Personal menus, on the other hand, are used for storing settings unique to the user and can only be interacted with by that user. However, it is still visible to other users as a simplified textured quad. These design choices have been made to ensure awareness of what the users are doing, as well as adding precision in communication by allowing users to interact with the same objects and to see any changes made by other users. Rays cast from the users' controllers, called pointers, helps to aid communication because it allows users to clearly highlight objects they are referring to.

\subsection{System Setup}

The RC System has been implemented using the Unity game engine, version 2018.3.11f. VR controller support has been added using the OpenVR library package in Unity, allowing for cross-platform support across different VR manufacturers. Required features not included with Unity were imported from external libraries. These include the Vive Media Decoder for video streaming and the ZED Mini SDK for the AR-HMD. Verbal communication was provided using an external solution (Skype). 
Unity has removed networking support in version 2018.3.11f while in the process of building a new solution. The networking in the RC System was ported to the Enet library [72] because it has a similar API to the old Unity networking system. Enet uses UDP with a Server-Client setup, and has been integrated to treat the local user as the server and the remote user as the client. Because the local user's computer is used as the server, it is used to store the true state of the system. Networking messages are sent with either reliable (i.e. object creation, etc.) or unreliable (for scene updates that quickly become obsolete) delivery depending on the use case. The server and client can disconnect if bandwidth becomes too large. To reduce this, updates are only sent from the server to the client every 100 milliseconds, with interpolation between updates performed on the client side. Data was also encoded using the bit-masking optimisation techniques mentioned in Section 2.5.

\subsection{User Setups}

\subsubsection{Gear Setup}

\section{Local User}

The local users view the shared space through an AR capable device with 6-DoF. Any AR capable device or HMD can be used; however, a video see-through HMD has been used to provide visual consistency between the local and remote users. This is done by attaching a ZED Mini camera to the front of the local user's HMD. Although the ZED Mini supports internal tracking, experimentation found issues with drifting, which was caused by the room used for testing. To solve this, a Vive Pro VR HMD is used along with the Vive tracking system for better tracking stability. The Vive Pro provides built-in video see-through support, however the ZED Mini is used because it has a significantly higher resolution and image quality. 
Unity support for the ZED Mini was imported using the official ZED Mini Unity SDK. The ZED Mini can scan a virtual representation of the real-world geometry for use in physics simulation or for shadow casting from virtual objects onto the real world. A limitation of this method is that it requires the use of the internal ZED Mini camera tracking to operate, which was found to suffer tracking drifting. To address this, the HMD tracking was used instead. Large planes representing the floor, walls and ceiling were manually placed by the user using the ZED Mini camera's depth map for correct alignment with the real-world geometry.

The ZED Mini SDK also uses the depth map to visually occlude virtual objects behind real-world objects. The accuracy of the depth map decreases with distance and suffers from noise. For this reason, the SDK has been modified to only occlude objects within a short distance from the ZED camera (i.e. the user's hands and controllers). Occlusion beyond this distance is handled using previously placed planes.

\section{Remote User}

The remote user can view the shared space through a VR HMD. During the testing of the system, an Oculus Rift was used for viewing VR content. Because the remote user views the shared space through a monoscopic 360 -video, movement is limited to rotation of 3-DoF. The 360-video is projected onto coarse geometry scanned and shared by the local user to help give a sense of depth, occlusion and parallax.

\subsubsection{0-Video}

\section{Live-Streaming to the Remote User}

A 360-camera is used to represent the remote user and to give the remote user an omni-directional view of the local space. The 360-camera is placed in the center of the local space at eye height, to simulate the feeling of being there, and is connected to the local PC. The 360 -video is streamed 
with RTMP over LAN to the client PC using an Nginx server running on the local PC. OBS Studio is used to stream the 360-video to Nginx. Video streaming and playback in Unity is handled using the Vive Media Decoder plugin [111], a wrapper of the FFmpeg media library for Unity, because Unity lacks the ability to stream RTMP videos. The plugin has been modified by enabling additional options provided by FFmpeg to optimize the plugin for lower latency live-streaming. Additionally, the plugin has been modified to display new video frames when possible, even though doing so results in inconsistent frame-rates. An in-depth breakdown of the setups for three different 360-cameras and the performance of each setup is discussed in Section 4.2.

\section{Streaming for lighting}

Beyond providing a view of the local space to the remote user, the 360video also provides lighting information to both users. The lighting for the virtual objects requires only a low resolution (512x256) 360-video. Because streaming the 360-video to both users increases the video latency, only the remote user receives the full resolution 360-video. The 360-video is compressed as a low-resolution image with JPEG encoding on the remote user's PC, before being forwarded onto the local user at roughly one frame per second. A detailed explanation for how the 360-video is used for lighting can be read in Section 3.6.

\subsubsection{Avatars}

Humanoid avatars are used to represent remote users to local users. The avatar setup also handles controller pointers and displays the simplified personal menu (Section 3.5.3). Because of this, avatars are used for both users but the actual avatar is kept hidden for the local user, who can already be seen in the 360-video. Models were generated from Adobe Fuse and automatically rigged using Adobe Mixamo. Unity provides inverse 
kinematics for humanoid rigs to realistically and automatically pose a model based on the positions and orientations of the head, hands and feet. The HMD and controllers were used to drive the values of the inverse kinematics rig. No devices were used for tracking the feet and attempts to approximate the feet positions resulted in the avatar poses feeling unnatural. To fix this, only the top half of the avatar is rendered. Using a better avatar representation has been left for future work.

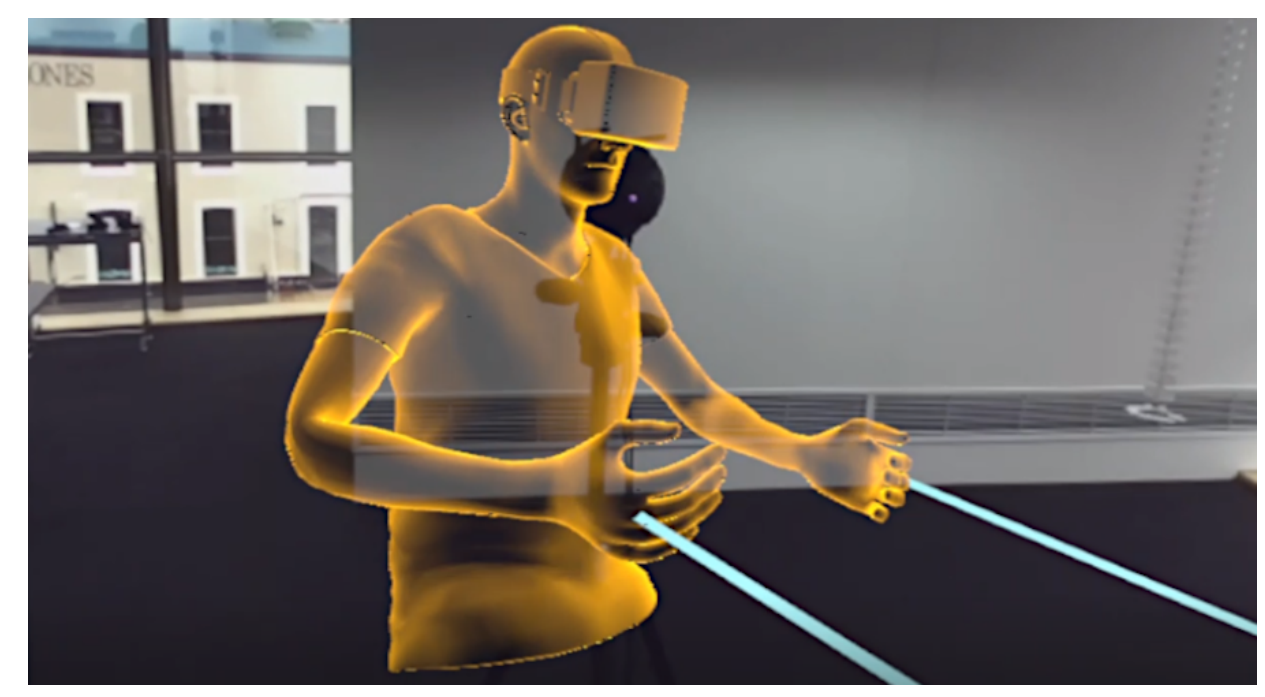

Figure 3.3: Remote user's avatar as seen from the Local User's perspective. The cyan lines (pointers) represent where the remote user is pointing.

Although previous related works show that realistic avatars provide better social-presence, there is the risk of highly-realistic humans looking too real and breaking presence, somewhat akin to the uncanny-valley effect. Some small tests revealed users found the realistic avatars to be unsettling. The avatars were abstracted to help overcome this issue. Instead of having realistic textures, the avatars were rendered as semi-transparent with a glowing outline to create a holographic like appearance. This was done as a way of keeping the full human body appearance without breaking immersion from semi-realistic shading. The avatar, as seen by the local user, is shown in Figure 3.3. 


\subsection{Shared Space}

The shared space represents the full working system where both users feel situated in the same physical space. Virtual elements, including virtual objects, user interfaces and avatars, are all components of the shared space. The space needs to be calibrated before being used so that the positions of virtual elements, in relation to the real-world, appear synced between users. Once the shared space is calibrated and synced, users can easily interact with objects and user interfaces, and experience face-to-face communication with each other.

\subsubsection{Space Calibration}

Because of different VR setups among users, each user should be synced with the shared space so that virtual objects appear in the correct realworld positions. Calibration is done in two stages: reconstructing 3D geometry of the local scene and matching the position and orientation of a virtual 360-camera with the physical 360-camera. The local space is assumed to be the true state of the system. Modifications to the virtual 360camera will affect the position and orientation of the remote user. Both users can manually adjust the virtual 360-camera to create a closer match between the virtual position and the physical world.

Geometry reconstruction of the local scene is also necessary for virtual objects physically interacting with the real-world. Although the ZEDMini supports geometry reconstruction, this feature requires ZED-Mini's inside-out tracking and does not work when only using the Vive tracking system. Instead, the depth estimation was used to manually place large planes around the space. This system provides a rough approximation for generating the walls, floor and ceiling. The scanned geometry also helps when calibrating the virtual 360-camera. The 360-video is projected onto scanned geometry whenever the local user modifies the virtual 360camera, appearing semi-transparently on top of the real-world space. 


\subsubsection{Objects}

The local and remote users can asynchronously interact with virtual objects, where each user takes turns to manipulate the object. Physics for the virtual objects are simulated using Unity3D's built-in physics engine. During user-to-object interactions, physics for the object is simulated on the local machine of the user in order to provide smoother, low-latency interaction for better precision when manipulating the object. Otherwise, the physics are simulated on the local user's server and synced with the remote user.

\subsubsection{User Interface}

The RC System provides two user interfaces to address the needs of the users. Shared menus are owner-less interfaces that all users can view and interact with. Personal menus are unique to each individual and are used to modify personal settings, appearing only as an abstract image to other users. Unity already offers a built-in solution for building user interfaces using components such as buttons, text boxes, toggles and scroll windows. These components have been extended to allow interaction from the VR controllers and were used to build the interfaces in the RC System.

\section{Personal Menus}

Menus may contain information specific to each user, including personal options and calibration settings. In these cases the only user who should be able to interact with the menu is the user directly affected. Displaying the settings in a shared menu breaks the user's privacy by allowing anyone to view and interact with it. Another option is to display the settings in a shared menu, but displayed differently for each user. However, this can confuse the users, who feel as though the other user is interacting with their own settings. Settings can also be different depending on the type of user. 
The solution to this problem is the introduction of personal menus. Each user is able to display their personal menu by holding the assigned button on their controller. Remote and local users have different menu options to suit their needs. As shown in Figure 3.4, the local user can use their personal menu to calibrate the shared space and scan real-world geometry. Remote users can change their avatar appearance, realign themselves to the shared space, and turn left and right without physically turning.

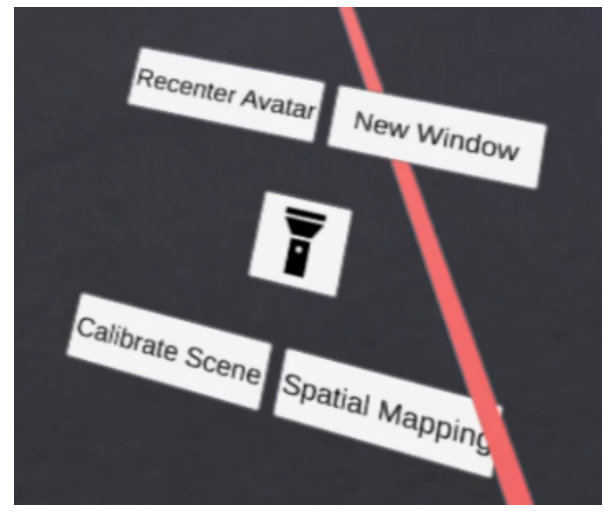

(a)

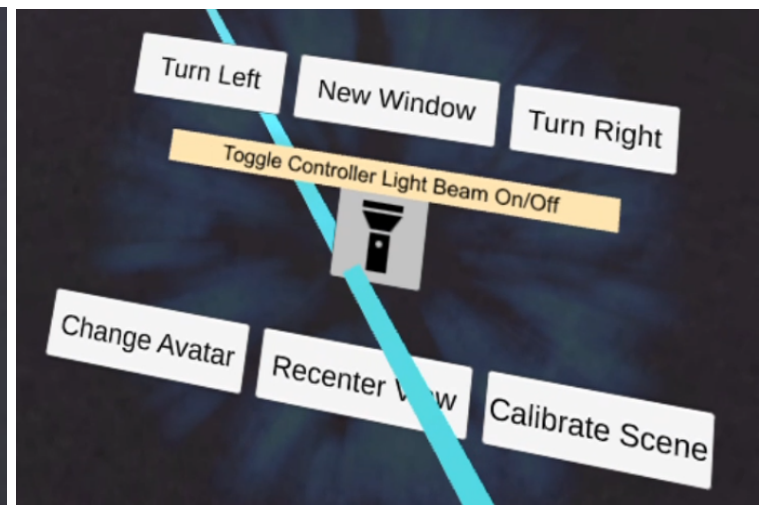

(b)

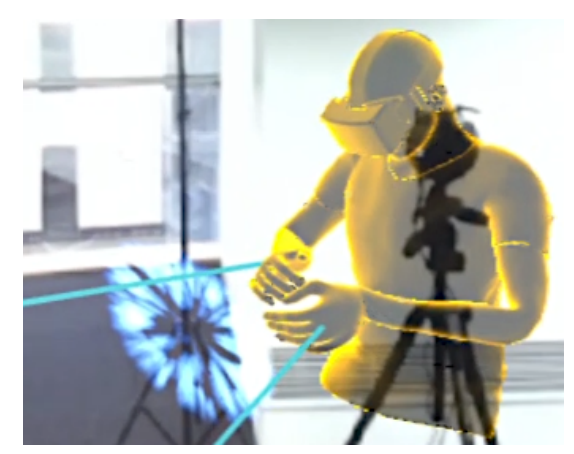

(c)

Figure 3.4: The personal menus for the local user (a), remote user (b), and as an abstract representation when viewed by a different user(c).

The personal menu appears in front of the controller and will realign in front of their controller if the user moves the controller too far away. The initial menu screen will only appear visible while the user is holding 
the "show menu" button on their VR controller. For convenience, sub menu screens (e.g. geometry scanning) will remain visible until manually closed by the user. Personal menus appear as an blue circular texture when viewed by other users (Figure 3.4(c)).

\section{Shared Menus}

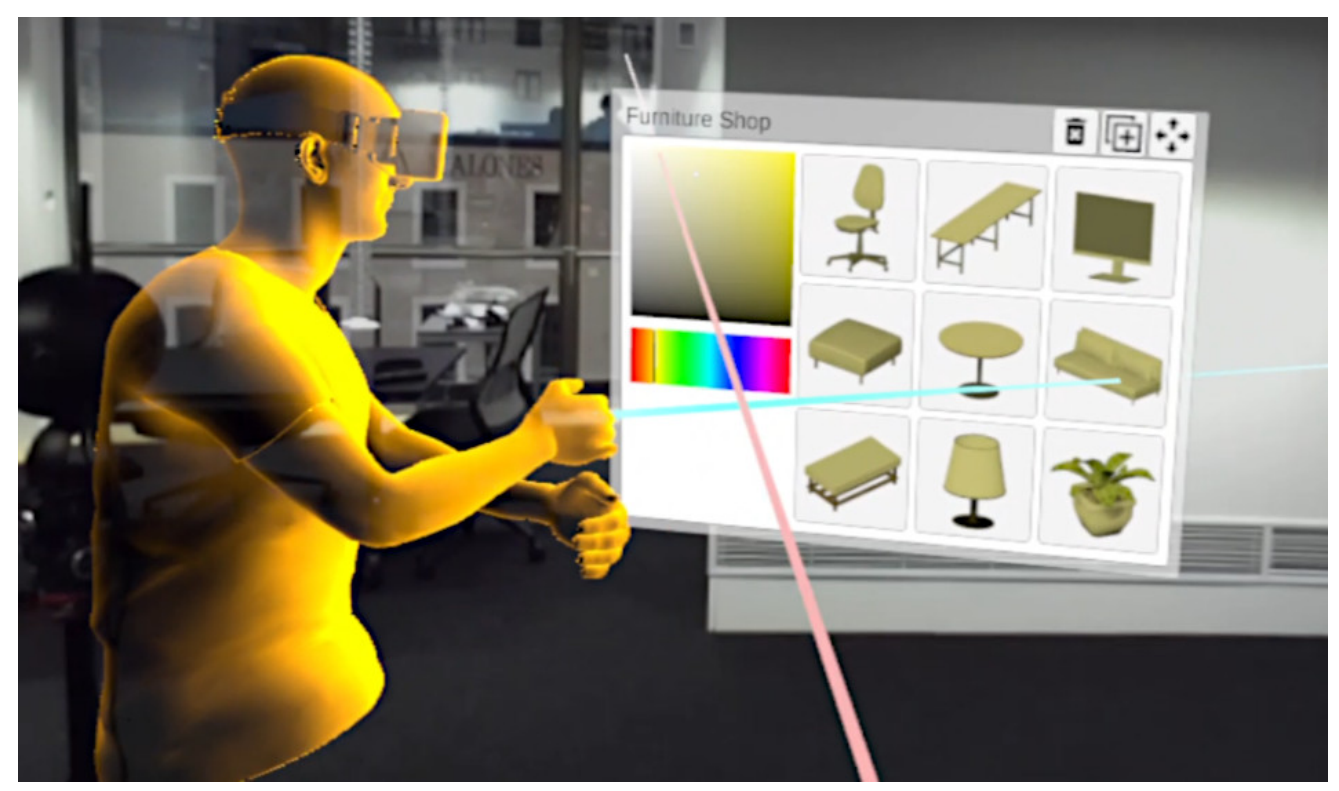

Figure 3.5: A remote user and local user interacting with a shared menu.

Shared menus (Figure 3.5) are windows floating in the shared space that all users can view and interact with. Users can move, duplicate or delete any shared menu. Users can also create a new shared window from their personal menu in cases where there are no shared windows already created or if the user finds it easier than duplicating a menu already in the scene. Unlike virtual objects in the shared space that update every frame, shared menus only change after user interaction. Shared menus are only synced over the network when modifications are made. 


\subsection{Mixed Reality Rendering}

Real-time rendering based on MR360 [87] has been incorporated into Unity to provide believable lighting and composition between virtual objects and the real-world scene. Both local and remote users utilise the 360-video as a source of ambient light and to detect real-world light sources used to cast shadows from the virtual objects onto the real-world. This implementation uses Unity's default forward rendering pipeline, which renders the virtual scene multiple times for each active light source and blends the results to create the final image (Figure 3.6).

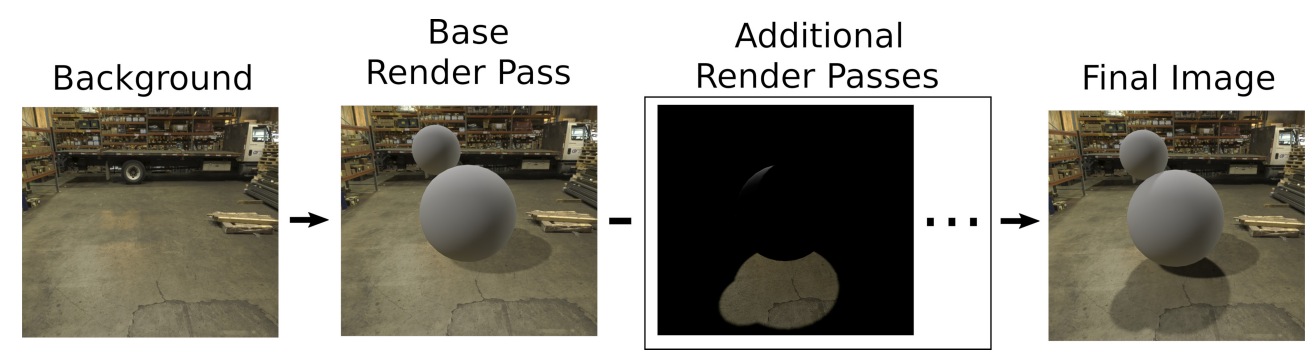

Figure 3.6: Render pipeline for forward differential rendering in Unity. The 360-image is taken from HDRIHaven. com under the CC0 licence.

\subsubsection{Lighting and Shading}

Image based lighting for simulating ambient light has been implemented using the method by Iorns et. al. [47]. Specular and diffuse light maps are computed simultaneously with a compute shader and stored in a single 3D texture. The inverse tone-mapped 360-video is used for purely reflective surfaces. Light detection has been implemented using the patch detection algorithm described by MR360 [87]. Patches of light are detected on the CPU, however, parallelism on the GPU is used for blurring the image and for calculating the mask of pixels that contribute to light sources. The 360-image is assumed to be stored on the GPU and is copied back to the CPU asynchronously to improve performance. 


\subsubsection{Shadow Catcher}

Virtual objects composited into real-world images should cast shadows to appear grounded in the image. This is usually done by using a virtual copy of the real-world geometry rendered with a material that captures and applies shadows cast from virtual objects onto the background image. A naive approach would be to apply the shadow by darkening the image where shadows are cast. However, physically accurate shadows that match the colour of the real-world shadows is possible. One such method that can realistically composite shadows into real-world images is Differential rendering [22].

Differential rendering works by rendering the shadow catching geometry with and without the virtual objects, and subtracting the difference between the two renders from the original image. This setup works well for offline rendering, which uses path-tracing and is not limited by the time to render the scene multiple times, but is not ideal for real-time performance. By default, Unity allows up to 4 shadow casting lights to be rendered and only casts shadows from the brightest light sources in the scene. Objects are rendered multiple times to account for each light source, an already costly operation. Furthermore, real-time rendering uses less accurate lighting estimation, which allows for optimisations in the shadow catching material. Real-time ray-tracing for more accurate lighting is now possible, but early support was only added to Unity late into the implementation of the RC System.

For the sake of simplicity, all surfaces in the real-world image $B$ are assumed to be fully diffuse. Using the lambertian reflectance model for rendering diffuse surfaces, the light hitting the surface can be defined as follows:

$$
B=\text { Albedo } \times\left(A_{L}+\sum_{i=0}^{n} L C_{i} \times L D_{i} \cdot N \times S_{i}\right)
$$


where the pixel colour is defined as the accumulation of ambient light $\left(A_{L}\right)$ and the sum of all light sources in the scene based on the light colour $(L C)$ and angle between the light direction $(L D)$ and surface normal $(N)$. $S_{i}$ denotes how much of the pixel is in shadow, ranging from fully in shadow (0) to no shadow (1). Finally, the light value is multiplied by the unlit surface texture (Albedo) to create the final pixel.

When adding shadows to a real-world image, the goal is to remove the effect of real-world light sources from areas receiving shadows. The colour, intensity and direction for each light source can be detected using the method described for MR360 [87]. Taking note of how differential rendering subtracts light based on shadowed and non-shadowed areas, the equation above can be reworked to account for subtracting the light affecting newly shadowed areas. The amount of light to remove from each render pass can be calculated as follows:

$$
\text { Output }_{r g b}=B \times L C_{i} \times\left(L D_{i} \cdot N\right) \times\left(1-S_{i}\right)
$$

The first render pass comprises of the background image with shadows cast from the brightest light source. Subsequent render passes can be subtracted using hardware blending. This equation also applies to diffuse lighting on virtual objects. The breakdown of each render pass is show in Figure 3.6.

The background image $B$ differs depending on whether the user is local or remote. For local users using an AR HMD, the background image can directly represent $B$. For the remote user, virtual objects composited into monoscopic 360-video will appear to float because of the stereo vision of HMDs. To fix this, the 360-video is projected from the position of the 360-camera onto the virtual geometry to give a rough sense of stereo depth (Figure 3.7). In this setup, $B$ represents the 360 -video as projected onto the geometry. A comparison of the final composition as viewed by the local and remote users can be seen in Figure 3.8. 


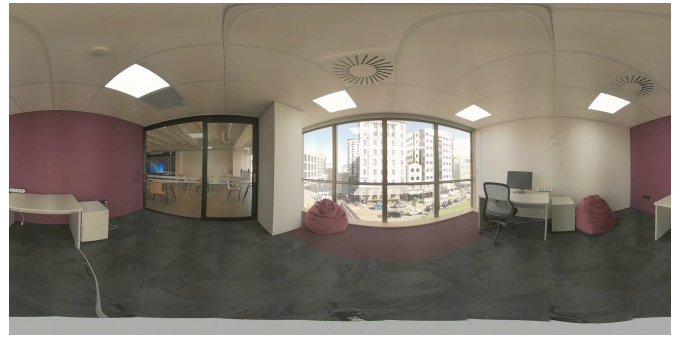

(a)

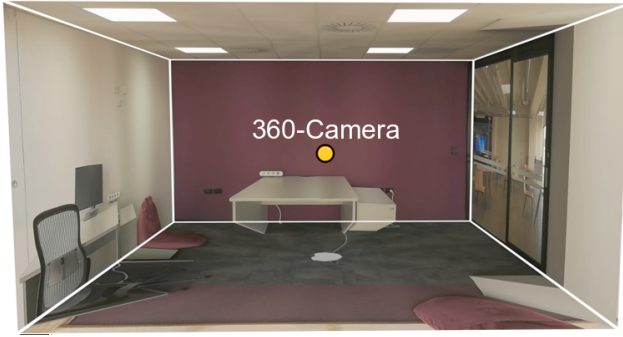

(b)

Figure 3.7: A 360-video (a) after it has been projected onto coarse room geometry (b). The yellow dot denotes the location of the 360-camera and the point from which the 360 -video is projected.

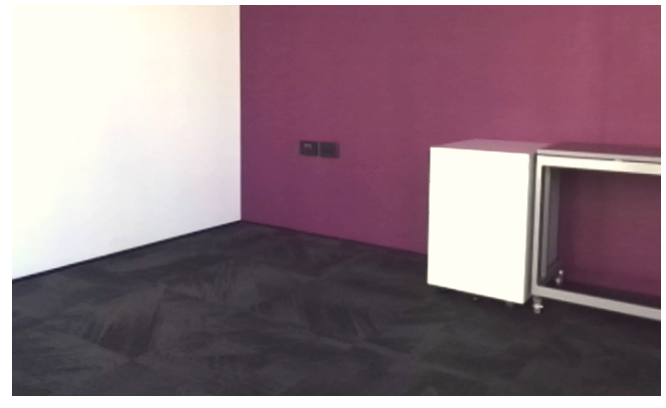

(a)

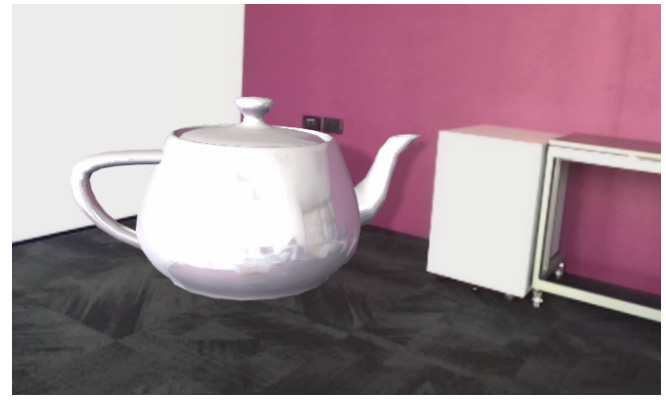

(c)

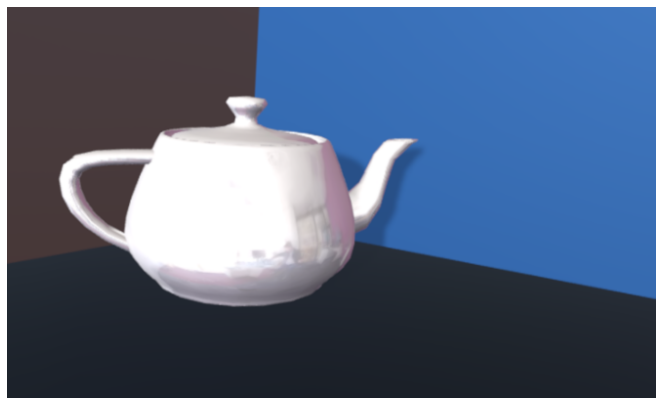

(b)

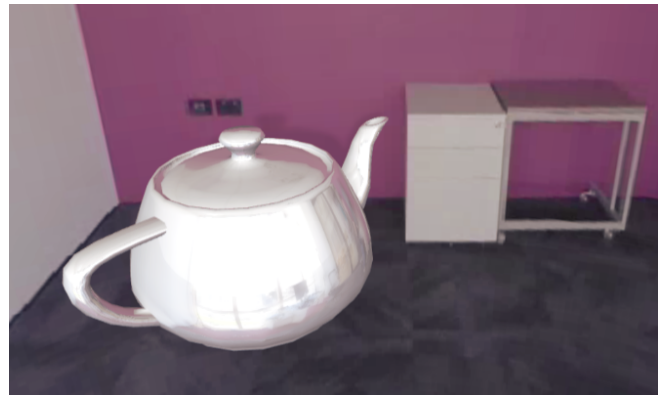

(d)

Figure 3.8: Rendering virtual objects into the real-world (a) using reconstructed geometry (b), and the final composition as seen by the local (c) and remote $(\mathrm{d})$ users. 


\subsection{Summary}

The RC System is implemented using the Unity game engine. The setup of the system allows a person in a remote space to feel teleported to a different "local" space and feel present with the person there. This is achieved by using a 360-camera placed in the local space that live-streams the location to the remote user wearing a VR HMD. The local user wears a AR HMD to see an avatar representing the remote user overlaid on the 360camera. The pose of the remote avatar is driven by the VR HMD and controllers of the remote user. The Vive Media Decoder with optimisations for live-streaming was used to receive the RTMP encoded 360-video in Unity a feature not supported by Unity. Networking for syncing avatars, objects, user interfaces and the low-resolution 360-video for lighting was done using a Unity wrapper of the Enet networking library, because of the lack of networking support in Unity at the time of implementation.

A key design decision of the system was to keep information transparent and shared between the users. The virtual space of each user is manually synced to create the shared space, so that objects will appear in the same real-world location to both users. The AR user can also recreate coarse geometry of the local space for use in physics simulation and casting shadows from virtual elements onto the real-world. Virtual objects controllable by any user are composited into each user's view and lit using the 360-video. Physics are simulated on the host PC unless the non-host user is interacting with the object, in which case that object is simulated on their PC to minimise latency.

Two user interfaces were incorporated into the system to handle scene calibration, user settings and virtual object creation. Personal settings only relevant to the individual user, such as avatar appearance, are stored within the personal menus, an interface attached to the VR controllers that can only be manipulated and viewed by the relevant user. Object creation and other tasks that are not user specific are delegated to the shared user 
interface, an owner-less floating window in the shared-space that can be controlled and seen by any user. With this setup of the RC System, users enjoy face-to-face communication, strong spatial awareness and presence, and a physical space enhanced with composited virtual elements. 


\section{Chapter 4}

\section{Evaluation and Results}

\subsection{Setup}

The RC System has been evaluated for the technical performance and user satisfaction. The same setup was used for both evaluations. While testing an earlier, simplified system, it was found that the remote user experienced different levels of presence depending on whether they were physically located in the same room as the local user (high presence) or in a different room (low presence). This observation drove the decision to split the local and remote users across two separated rooms with no ability to see each other outside of the desired visibility provided by the system. The goal was to test whether the system could provide high presence for the worst test-condition. The layout of the rooms where the local and remote space were set up can be seen in Figure 4.1.

Both users view the shared space through VR HMDs. This ensured a similar visual quality, field of view and refresh rate between both users. The local user's computer used an Intel Xeon Gold 5122 CPU, Nvidia Quadro P5000 GPU and 32GB RAM. A laptop was used for the remote user and had an Intel Core I7-6700HQ CPU, Nvidia GTX 980M and 16GB RAM. The setup was tested over a local area network (LAN) wired with 1Gbps Cat6 Ethernet cable. 


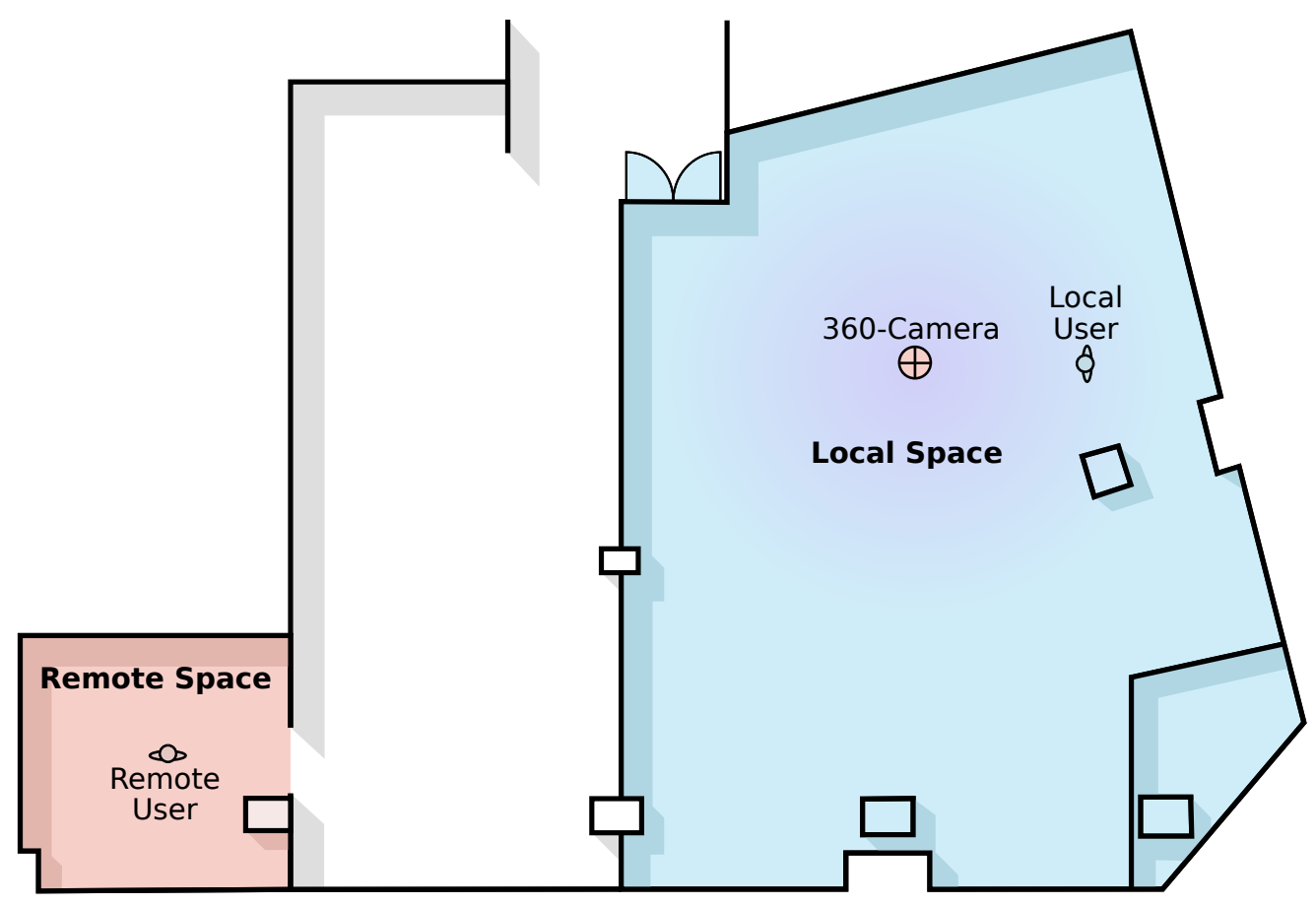

(a)

Figure 4.1: The floor plan during the evaluation.

The local user used the HTC Vive Pro and Vive controllers with the Vive tracking system for full 6-DoF. Although the HTC Vive Pro supports video see-through, the resolution per eye is low $(612 \times 480)$. A ZED Mini camera was attached to the front to provide higher resolution video seethrough. The ZED Mini camera was streamed at 1280x720 resolution per eye at $60 \mathrm{~Hz}$.

The remote user used an an Oculus Rift CV1 HMD to view the shared space. Although the Oculus Rift supports full 6-DoF, the 360-video only provides 3-DoF. During pilot testing, participants felt compelled to walk around the local space, especially when trying to get closer to objects to pick them up. Because the 360-video lacks positional freedom, remote users were seated while performing the tasks to discourage movement. 


\subsection{0-Camera Performance}

Three 360-cameras (Ricoh Theta V, Samsung 360 Round, Insta360 Pro 2) were compared based on visual quality and latency when streaming over a LAN. The camera with the best overall visual quality and latency was chosen for the user evaluation. All cameras were streamed over the network using an Nginx RTMP server running on the local user's PC and H.264 encoding at $4000 \mathrm{kbps}$. OBS Studio was used to encode and stream the video footage to Nginx for the Insta360 Pro 2 and Ricoh Theta V. The Samsung 360 Round can stream directly to Nginx, so OBS Studio was not required. Videos streamed with OBS Studio were encoded using the x264 library. Parameters were set as: (presets: ultrafast, profile: baseline, tune: zerolatency). The video was streamed into Unity using the modified Vive Media Decoder described in Section 3.4.2. Latency for streaming into FFplay, the media player that ships with FFmpeg, was tested as well for reference, using FFplay's default parameters and with the nobuffer flag enabled. Figure 4.2 shows a diagram overview of each camera setup.

\subsubsection{Results}

\section{Visual Quality}

Visual quality between the 360-cameras was evaluated by the author observing the stitching quality and colour accuracy. For image quality, the Insta360 Pro 2 and Samsung 360 Round provide similar levels of quality and dynamic range. Several settings, including exposure, saturation and brightness, can be tuned using the proprietary software to better capture the lighting conditions of the environment. The Ricoh Theta $V$ can only live-stream with a low dynamic range, leading to a loss of detail in over or under-exposed areas. Experimenting with Ricoh's mobile-based app found control of the exposure and colour is only supported for photos or recorded videos. 


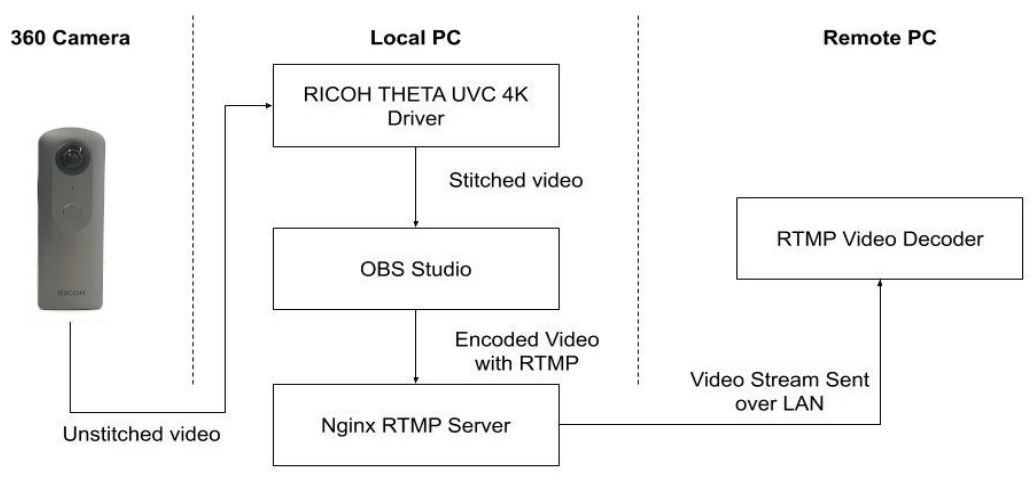

(a) Setup for the Ricoh Theta V.

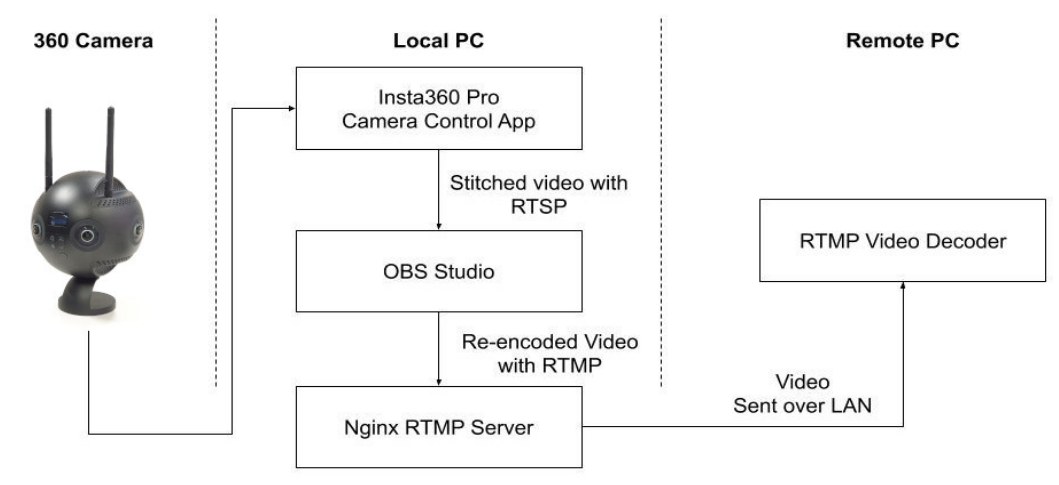

(b) Setup for the Inst360 Pro 2.

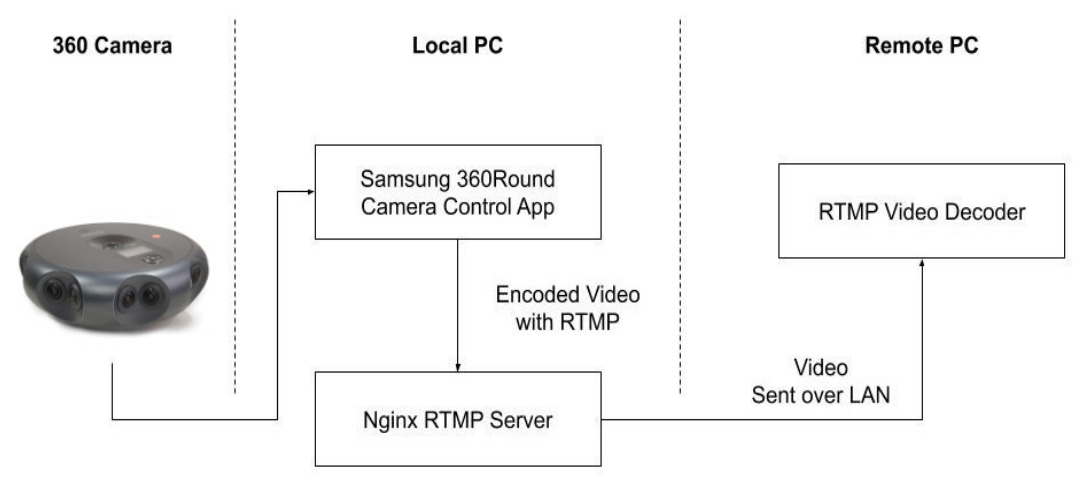

(c) Setup for the Samsung 360 Round.

Figure 4.2: The setups for streaming videos captured with the 360 cameras to a different computer (Remote PC) over LAN. 
For 360-video stitching, the Samsung 360 Round provides the best result with only slight image distortion appearing at the seams. The Insta360 Pro 2 and Ricoh Theta V linearly blends seams together causing ghosting artefacts. The outputs from the three cameras can be seen in Figure 4.3, along with some highlighted stitching artefacts.

\section{Latency}

Approximate latency was recorded when the 360-video is first viewable on the local computer (OBS Studio for Ricoh Theta and proprietary software for Insta360 Pro 2 and Samsung 360 Round) and when viewed on the remote computer in Unity or FFPlay. Latency was measured by performing an action in front of the camera (a person raising their hand) and measuring the time with a stopwatch until that action appeared in the video. This procedure was repeated 10 times and averaged to get the approximate latency. The video stream was restarted for each of the 10 times. The optimised video decoder in Unity was able to stream with minimal latency in most cases, however, it did become unstable and perform poorly for larger resolutions or videos not encoded in OBS Studio (as seen in Table 4.1 with the $4 \mathrm{~K}$ Samsung 360 Round streamed to Unity compared to the latency displayed in FFplay). Although it can be improved, the method used for measuring latency did yield the same result for the Samsung 360 Round preview as the official latency ${ }^{1}$. Expected latency as reported by the manufactures of the Ricoh Theta V and Insta360 Pro 2 was not found.

Based on the results shown in Table 4.1 and the visual quality analysis, Insta360 Pro 2 was chosen for the setup as the best compromise between visual quality and low latency. The video was streamed at HD resolution instead of $4 \mathrm{~K}$ for improved stability during the user study testing.

\footnotetext{
${ }^{1}$ https://image-us.samsung.com/Samsungus/home/mobile/ virtual-reality/pdp/sm-r260nzkaxar/WER-360ROUNDDSHT-DEC17T_ Final_12-14-17.pdf
} 

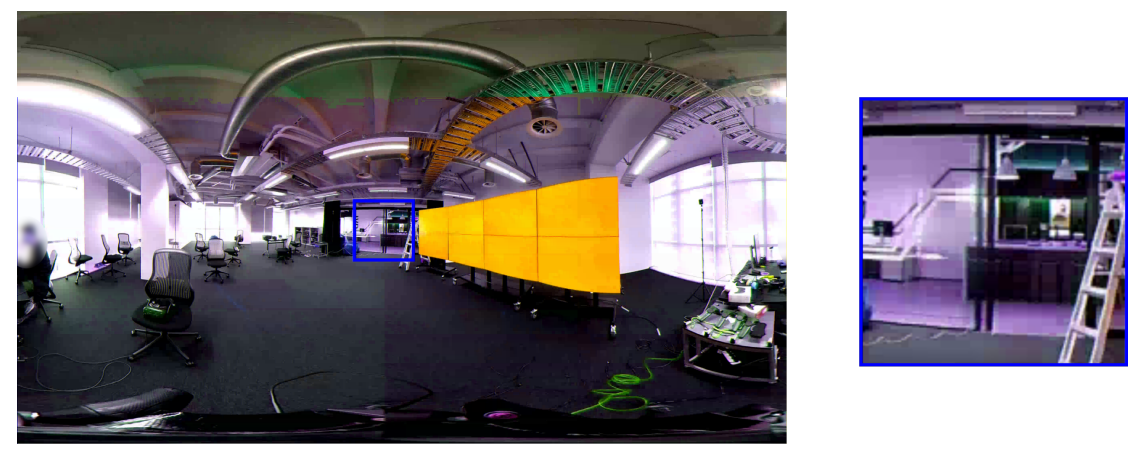

(a) Ricoh Theta V
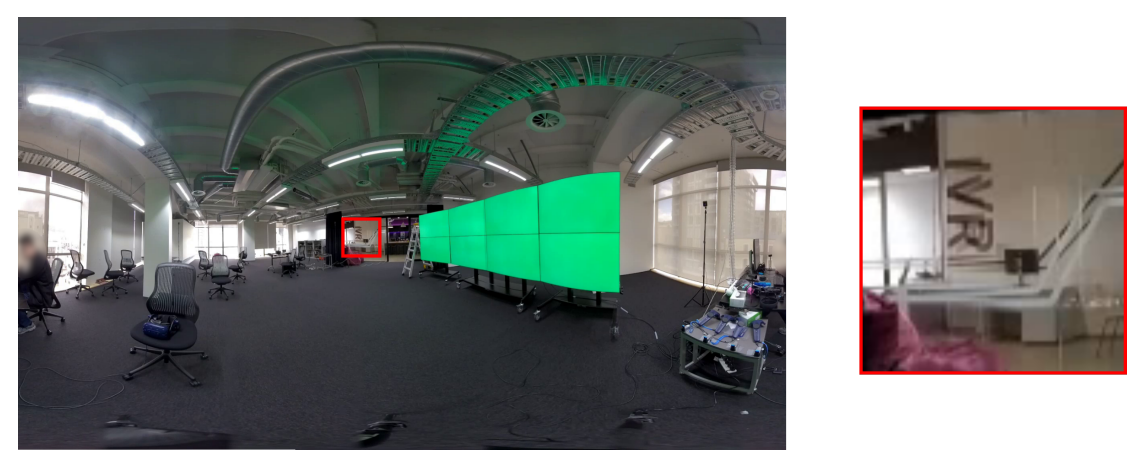

(b) Insta360 Pro 2
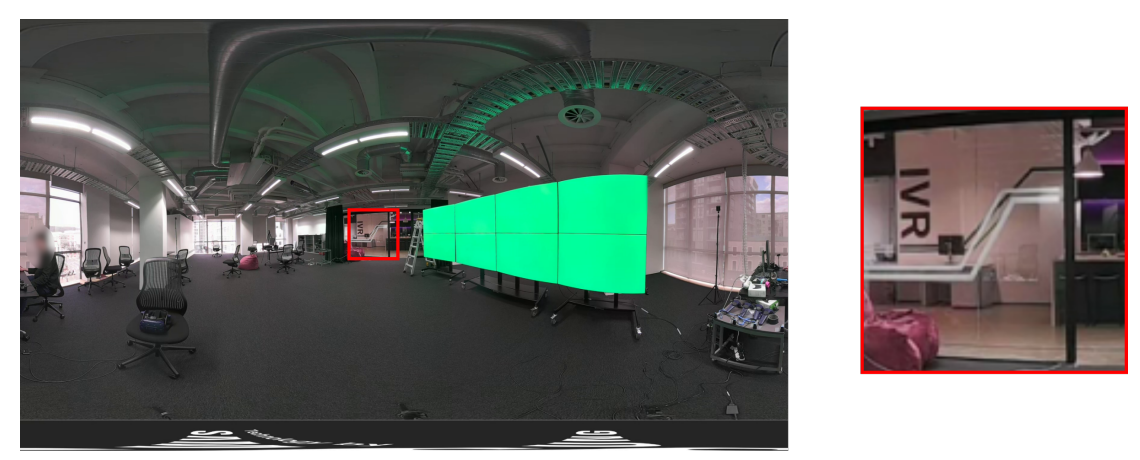

(c) Samsung 360 Round

Figure 4.3: Image comparison between the 360-cameras. Please note the better dynamic range and more natural colours seen in (b) and (c), and the stitching artefacts seen in the ghosting on the door (a) and ghosting on the IVR text (b). 


\begin{tabular}{|r||cc|cc|cc|}
\hline \multicolumn{1}{|c||}{ Camera } & \multicolumn{2}{c|}{$\begin{array}{c}\text { Ricoh } \\
\text { Theta V }\end{array}$} & \multicolumn{2}{c|}{$\begin{array}{c}\text { Insta360 } \\
\text { Pro 2 }\end{array}$} & \multicolumn{2}{c|}{$\begin{array}{c}\text { Samsung } \\
360 \text { Round }\end{array}$} \\
\hline Resolution & HD & $4 \mathrm{~K}$ & HD & $4 \mathrm{~K}$ & $2 \mathrm{~K}$ & $4 \mathrm{~K}$ \\
\hline Preview & $0.5 \mathrm{~s}$ & $0.7 \mathrm{~s}$ & $0.6 \mathrm{~s}$ & $0.6 \mathrm{~s}$ & $1.7 \mathrm{~s}$ & $1.7 \mathrm{~s}$ \\
Unity & $0.6 \mathrm{~s}$ & $0.8 \mathrm{~s}$ & $1.2 \mathrm{~s}$ & $1.2 \mathrm{~s}$ & $1.7 \mathrm{~s}$ & $10.5 \mathrm{~s}$ \\
FFplay & $0.8 \mathrm{~s}$ & $1.2 \mathrm{~s}$ & $1.5 \mathrm{~s}$ & $1.4 \mathrm{~s}$ & $2.1 \mathrm{~s}$ & $2.1 \mathrm{~s}$ \\
\hline
\end{tabular}

Table 4.1: Approximate latency at different stages of the setup for each camera.

\subsection{User Study}

A user study was conducted to evaluate the RC System for user satisfaction, presence and collaboration. A total of 20 pairs (40 people) were recruited for the user study, three of whom had not previously met. During the test, pairs were split between two rooms (local space and remote space) when performing the tasks and answering the questions. One participant would assume the role of the local user while the other participant would act as the remote user. The user study was conducted following the guidelines laid out in the ethics form, application number: 0000026514.

\subsubsection{Methodology}

The study was broken into three overarching stages performed in the order of introduction, performing the tasks and providing feedback. Times and stages were broken down approximately as follows:

\begin{tabular}{|c||c|c|c|c|c|}
\hline Stage & Briefing & Task 1 & Task2 & Questionnaires & Interview \\
\hline Time & $10 \mathrm{mins}$ & $10 \mathrm{mins}$ & $10 \mathrm{mins}$ & $20 \mathrm{mins}$ & $5 \mathrm{mins}$ \\
\hline
\end{tabular}

Table 4.2: Tasks and Approximate Times 
Participants were asked to sign a consent form (Appendix B) and read an information sheet (Appendix C) before starting the user study. They were then shown the setup of the local space, explained the goals of the system and briefed on the tasks, controls, interactions and interfaces (Appendix A). The pair decided who would assume the role of the local user and remote user. Upon deciding, the pair was broken up and taken to their respective rooms. Moderators were located in both locations to help participants use the system and to answer any questions.

Participants were given two tasks to perform. During the tasks participants could communicate verbally and with gestures, including pointing cues and head gaze. The first task was designed to train participants to use the system. Participants could ask questions during this task and moderators could give advice. The study moved onto the second task once participants indicated they were comfortable with the system. During the second task, communication between the participants and moderators was kept minimal, with communication only occurring if participants were unclear on how to perform the task or when participants were satisfied they had completed the task. The remote and local spaces were calibrated by the moderators prior to the participants using the system.

After completing both tasks, participants were asked to fill in two sets of questionnaires: one for gathering general information of the participants and the other for providing feedback. A quantitative evaluation of presence and collaboration was gathered using the SoPQ (Spatial Presence Questionnaire) [36] and MEC-SPQ [113] questionnaire. Additional questions were included to assess parts of the the system not covered by the previous questionnaires. These questions were a part of a collaborative effort and are only summarised in this thesis. The questions used during the study have been included in Appendix D. Please refer to the work of Rhee et al [88] for a full evaluation of the questionnaires. Users also provided written feedback to several custom questions, which is covered in Section 4.4.2. 


\subsubsection{Tasks}

The first task (Figure 4.4) participants performed was designed to train the participants to use the system. Participants were provided 9 furniture models and given the freedom to decorate the shared space to their liking. Participants were not tested on any point and there were no goals during this task. Roughly 10 minutes was given to participants to learn the system before moving onto the second task.

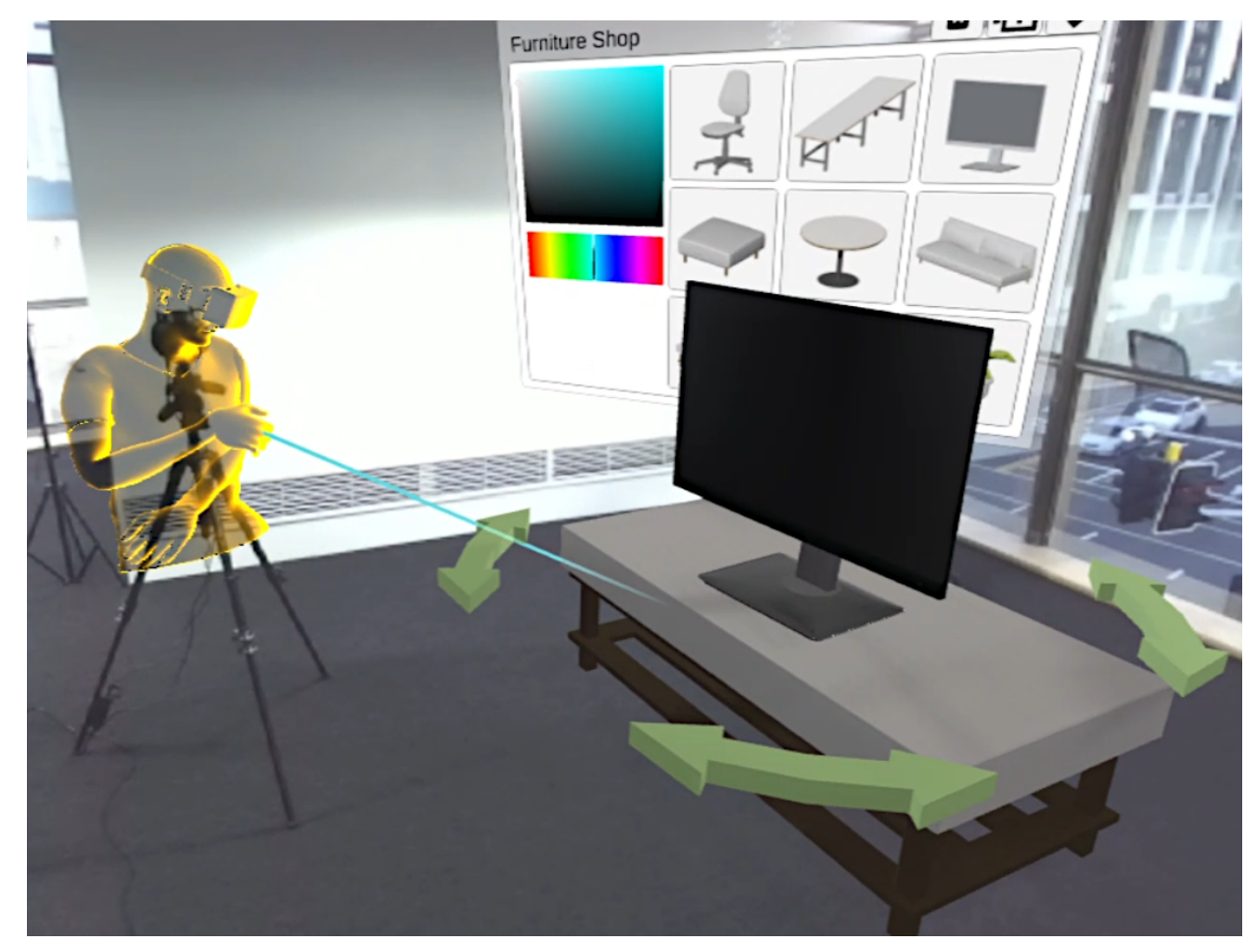

Figure 4.4: Training task.

The second task (Figure 4.5) was designed to test how well users could collaborate and communicate in the system. This task was similar to the training task, except participants were given a layout of where to place objects. The interface was also simplified to only include 6 shapes and 8 colours. Each participant was assigned 3 objects to place in the shared 
space, and the task was completed once the participants were satisfied they had correctly placed all 6 objects. A blueprint of the shared space was provided to each user, showing which objects to place where, how they should be rotated and what colour to use. Participants could only see the blueprint for the other user and had to guide them to place the objects correctly.

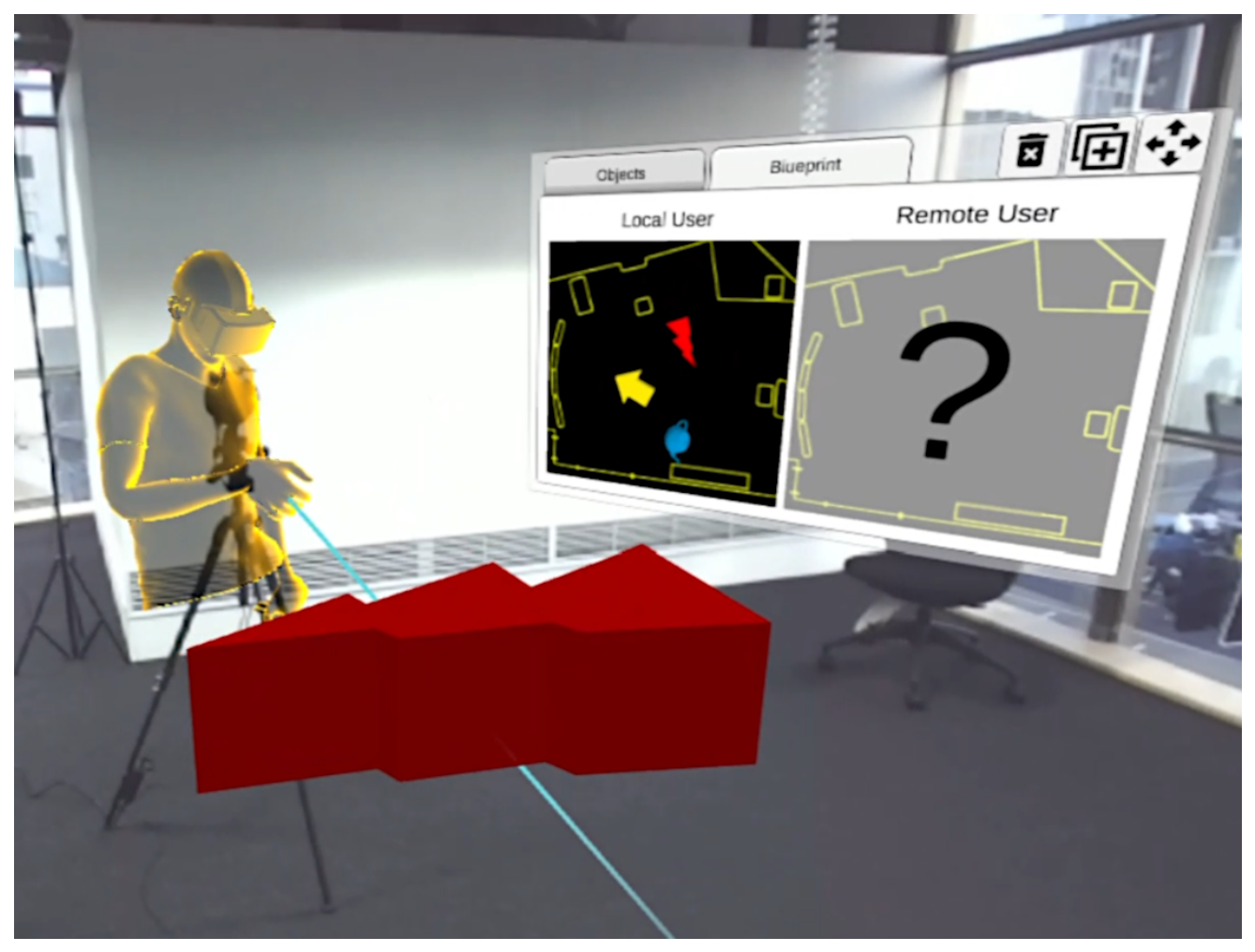

Figure 4.5: Main task.

Users were provided similar interfaces for both tasks, as shown in Figure 4.6. A shared window is provided for the training task that includes a colour editor and a selection of furniture models. Once a colour is selected, participants can create and move the virtual objects using the VR controllers with a click and drag mechanic, and rotate by clicking and dragging arrows that appear around the object (see Figure 4.4). 


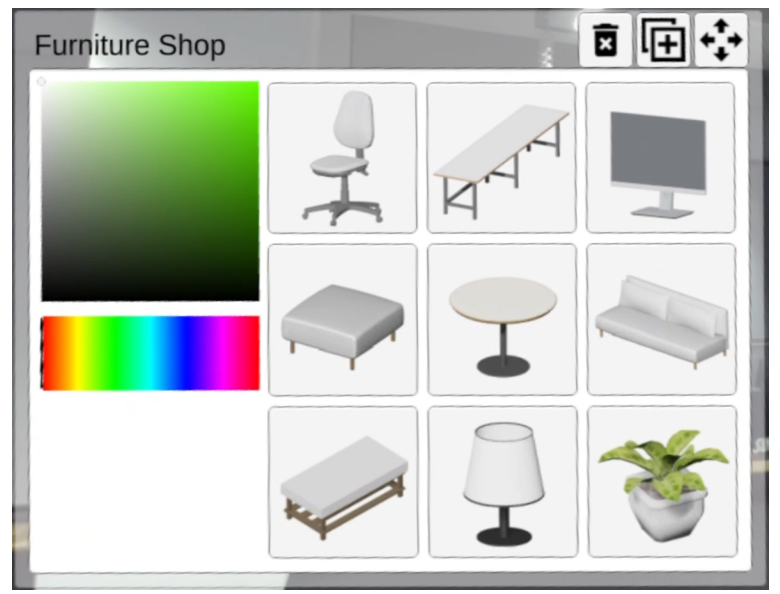

(a) Shared menu for the training task.
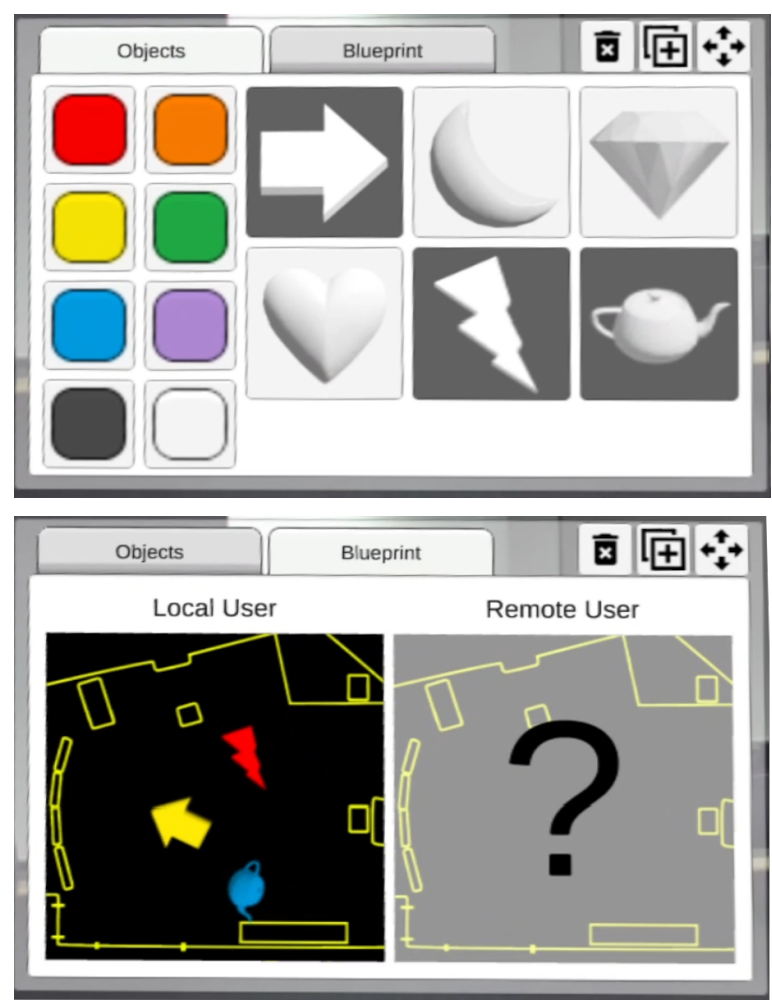

(b) Shared menu for the main task, showing the Objects tab (top) and Blueprint tab (bottom).

Figure 4.6: Shared menu during the training task (a) and main task (b). 
A similar interface is provided for the main task. However, the colour editor was simplified to 8 colours in the shared window for user convenience. Some objects were greyed out so that participants could only place objects assigned to them. A new tab was included for viewing a blueprint of where the virtual objects should be placed in the room. Additionally, users could view a copy of their blueprint in their personal menus.

\section{$4.4 \quad$ Results}

A total of 40 participants between the ages of 18 and 64 were recruited for the study, with $62.5 \%$ being between 20 to 30 years of age. Participants were recruited from different schools within the university and from external businesses. The majority of participants had little to no experience in VR or AR, and only minor experience viewing 360-videos. Experience with traditional software (i.e., video-chat applications, games and 3D applications) was much more varied. Results were recorded through tracking data during the evaluation and written text based feedback.

\subsubsection{Recorded Data}

Data was recorded while participants performed the second task of placing shapes around the room. Recorded information included the users' solutions to the tasks, and the networking bandwidth used by the system during the study. Because positions and orientations of the objects in the users' solutions had to be manually matched to the actual answer, the distances and orientations of the shapes are approximates rather than absolutes.

Participants spent an average of 8.6 minutes (2.1SD) on the second task. Objects were placed an average of 0.8 meters (0.1 SD) away from the correct positions, with the average orientation of the objects being off by $12.2^{\circ}$ (7.0 SD). No correlation was found between the time participants spent on 
the task and the accuracy of the results. The approximated accuracy for each object can be seen in Table 4.3

On average, data was sent across the network at a rate of $8.99 \mathrm{KBps}$ (0.47SD, Kilobytes per second). Of this, $1.12 \mathrm{KBps}(0.22 \mathrm{SD})$ was sent by the local user and $0.98 \mathrm{KBps}(0.19 \mathrm{SD})$ was sent by the remote user. The remaining $6.90 \mathrm{KBps}(0.47 \mathrm{SD})$ were used for sending the low-resolution video stream from the remote user to the local user. This information only includes data sent within the system and does not include the bandwidth used to stream the full $4 \mathrm{~K}$ video nor verbal communication.

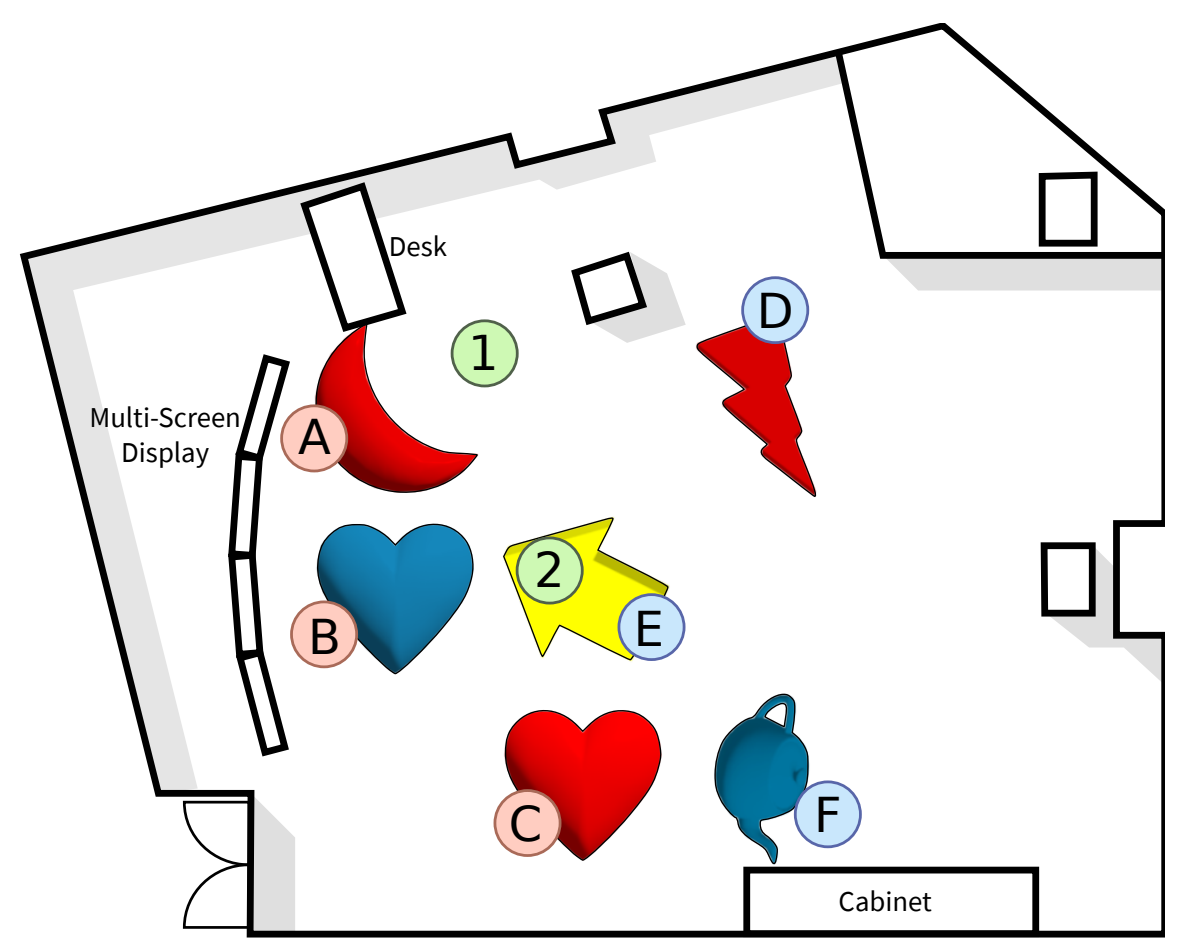

Figure 4.7: Room and shape layout for the second task. Shapes A, B and $C$ were placed by the local user. The remaining shapes were placed by the remote user. Positions 1 and 2 denote where the local user and 360-camera were positioned. 


\begin{tabular}{|c||c|c|c|c|c|c|}
\hline Shape & A & B & C & D & E & F \\
\hline Position & $0.5 \mathrm{~m}$ & $0.5 \mathrm{~m}$ & $1.2 \mathrm{~m}$ & $0.9 \mathrm{~m}$ & $0.8 \mathrm{~m}$ & $0.6 \mathrm{~m}$ \\
& $(0.3 \mathrm{SD})$ & $(0.3 \mathrm{SD})$ & $(0.5 \mathrm{SD})$ & $(0.3 \mathrm{SD})$ & $(0.3 \mathrm{SD})$ & $(0.3 \mathrm{SD})$ \\
\hline Angle & $14.5^{\circ}$ & $11.4^{\circ}$ & $9.2^{\circ}$ & $11.7^{\circ}$ & $17.4^{\circ}$ & $9.4^{\circ}$ \\
& $(5.8 \mathrm{SD})$ & $(6.2 \mathrm{SD})$ & $(6.6 \mathrm{SD})$ & $(4.5 \mathrm{SD})$ & $(8.5 \mathrm{SD})$ & $(6.2 \mathrm{SD})$ \\
\hline
\end{tabular}

Table 4.3: Average distances and angle offsets from the expected values for each of the shapes shown in Figure 4.7.

\subsubsection{Feedback}

Participants were asked to provide written feedback of the RC System. Throughout this feedback summary, Local and Remote denotes whether feedback came from a local or remote user, followed by their written feedback. The questions asked were as follows:

- Were there any factors that made communication easier or harder?

- What were some factors that helped or hindered your collaboration?

- What were some factors that made you feel present with the other user?

- What did you like about the system?

- How would you improve the system?

The feedback for the concept of the system and the experience of using the system for collaboration and communication was positive. Many of the participants found the system easy to use and had fun partaking in the study. Communication felt natural and they enjoyed being able to talk and use gestures, head gaze and the controller pointers to clarify their communication. The ability to mimic face to face communication made the experience feel more natural. 
Local: "Voice chat and seeing where my partner is, what he is doing or where is he pointing made the communication much easier.".

Participants appreciated being able to see and interact with the same virtual objects and interfaces.

Remote: "I liked the shared UI where both my partner and I can use.". Local: "That we could interact with the same objects really made it feel like we were in the same place.".

Sharing the same real-world space allowed participants to be able to make reference to real-world objects located in the space.

Remote: "Referencing object in the room makes communication easier, for instance the computer desk is a good reference.".

Local: "That he responded correctly to the verbal directions I used to describe locations in the local space made it seem like we must be in the same place/seeing the same thing.".

In fact, some of the remote participants mentioned becoming so immersed in the system that they felt present in the local space.

Remote: "I actually forgot for a moment that I was in another room".

Remote: "I could see the other place like I was there".

Although the 360-video had over a second of latency, no comments were made regarding this. The resolution of the AR view and 360-video was mentioned as being low. Shadows cast from the virtual objects were helpful during the collaborative task. However, the overall fidelity was mentioned as an area of improvement. 
Local: "If using AR space, using actual images of objects, if possible, rather than digital rendering, would be much more immersive and representative.".

Lack of proper real-world depth was also reported. Remote users appreciated a sense of depth when objects were placed in the room. However, this illusion broke when viewing virtual objects placed next to real-world objects in the 2D 360-video. Using 3D 360-video was proposed as an improvement by some remote users.

Remote: "A sense of depth of the other person would be nice, since it becomes clear that they are only $2 D$ when objects are placed near them.".

Participants wanted more freedom of movement to walk around the room. The local users were restricted to moving only as far as the wired HMD allowed. The remote users could not move in the shared space because the 360-video was stationary. Remote users were seated during the test to discourage trying to walk within the 360 -video, a possibility seen during pilot tests. However, they still expressed a desire to do so.

Local: "Wires on back of head; how far can I move around??".

Remote: "I found it hard being in the remote room to move around the other room being on the chair".

The limited tracking range for the remote users was also mentioned as damaging immersion.

Remote: "Not being able to turn my head too far without losing connection to the Oculus hurt my immersion somewhat.".

Local users appreciated the presence provided by seeing an avatar representation of the remote user. However, they also expressed preference in seeing an improved full-body avatar representation of the remote user. 
Local: "The ability to see the direction my partner was facing and where their arms were facing made them feel present in the space".

Local: "I would add a full body avatar to increase feeling of interaction and co-presence.".

A problem noted during the study and commented on by participants was the inaccurate calibration between the local and remote users. For the remote users, the controller beams of the local users did not always align with the person seen in the 360-video.

Remote: "It would also be nice if the beams from the AR person's controllers matched with the actual controllers in their hands.".

Likewise, virtual objects did not always appear in the exact same position in the room when viewed by different users. The lack of accuracy in calibration reduced confidence and immersion in the system.

Remote: "The beams of light did not match up exactly with the camera image I could see of the other person, so I was not entirely confident that they were ending in the correct place either.".

The system itself, although well received, had suggestions for refining the experience. The controllers could be improved through tighter controls in manipulation of objects. One suggestion mentioned using vibration in the controller to provide feedback when the virtual object being held was interacting with the floor. A remote user expressed a desire to use hand gestures instead of controllers, an option that can be accomplished using a sensor such as the Leap Motion controller ${ }^{2}$.

Remote: "I felt like i wanted to communicate with my hands but because I was holding the VR remotes my communication was limited.".

\footnotetext{
${ }^{2}$ https: / / www.ultraleap.com/
} 
People generally had little trouble with the interface, however, some objects were hard to delete and people had trouble placing windows in a area that suited both users.

Local: "When deleting, if the area tolerance for applying a delete was larger, and the object also flashed or went a certain colour when it was in the deletion range.".

Remote: "NOT ABLE to easily align windows to suitable orientation, other partners objects occluding windows.".

Improved sound was suggested by having sounds play when objects collide and using 3D positional sound.

Remote: "It would be good if we could hear the sound when the object fell on the ground".

Remote: "directional sound".

\subsection{Discussion}

The goal of the RC System is to simulate face-to-face collaboration as if all users are physically present in the same location, even when not. To achieve this goal, there are several sub-goals that should be met. The visual quality should achieve high fidelity between the virtual objects and real-world. Remote and local users should feel a high sense of socialpresence as if they were in the same room. Similarly, the remote user should feel as if they have transported from their location to the local space. The system should provide a platform for efficient collaboration and communication. Finally, all interactions should occur in real-time, with minimal latency affecting the interaction. 


\subsubsection{User Presence}

Presence felt by the users was evaluated using a questionnaire (Appendix D). This questionnaire was analysed using the Mann-Whitney $U$ test to find if there were any statistical differences in the presence felt between the AR and VR user. The test failed to show any statistical significance. Two more statistical tests, a two-one-sided t-test (TOST) analysis and the Wilcoxon Signed Ranks test, were performed to test for equivalence between the VR and AR users groups. The results found that both AR and VR users experienced similarly high levels of presence when using the system. The full explanation, analysis and breakdown of the results is discussed in-depth in the work by Rhee. et. al [88].

\subsubsection{User Satisfaction and Performance}

The system was well received by participants, who reported finding the system fun and easy to use. Users enjoyed being able to see each other and interact with the same objects and shared interfaces. Communication within the system felt natural to the users. It was observed that, while completing the tasks, users communicated with face-to-face communication as if they were both physically co-present. It can be concluded that as a concept, the system succeeds in the key points of providing natural communication and collaboration regardless of the users' locations.

Some remote users even reported becoming immersed in the system to the point of becoming more aware of the local space than their physical space. An early prototype of the system was not able to create any sense of tele-presence. A possible reason why the RC System succeeded where the early prototype failed could be in the level of shared interactions. The early prototype used an asynchronous setup, allowing the remote user to interact with virtual objects and to view the shared space through a live-streaming 360-video. However, the local user could only view the shared space on a monitor and had no ability to interact. The RC System's 
setup was kept synchronous between users as often as possible. Both users could see the same objects, interfaces, interactions and each other located in the same space. Users praised the system for this ability through their feedback. User feedback also suggested the 360-camera representing the remote user helped improve social-presence and tele-presence, by giving the remote user a real-world view of the local-space and allowing highfidelity face-to-face communication.

The calibration of the system was a major issue raised by participants and noted during the evaluation. The difference between what each user saw reduced the sense of sharing the same space and caused frustration when the participants were aiming for high accuracy when completing the tasks. Improved audio was also mentioned as an improvement to make the system more immersive. 3D voice communication was explored but eventually rejected for an external solution after experiencing issues of corrupting audio and unstable network connections. Added sounds effects were not explored, but provides an area for future work.

Shadows cast from the virtual objects onto the real-world was mentioned as being beneficial to having objects feel grounded in the real-world. However, the overall visual quality of the system can be improved. User feedback suggested that the virtual objects did not achieve high realism. Interestingly, these comments came mainly from the local users. The AR see-through camera automatically adjusts the exposure of the image to match the brightness of the view in front of the user. Lighting of the virtual objects does not change brightness to reflect this, leading to possible mis-matching in lighting between the virtual and physical space.

Other aspects of the visual quality that received negative responses from participants can be improved with further development or hardware improvements. The resolution of the 360-video, AR see-through camera and HMDs will increase as hardware improves. Local users also expressed a preference for seeing a better avatar representation of the remote user. Research into better avatar representations already exists and further de- 
velopment would enable these solutions to be incorporated into the system. Improved avatar representation and visual resolution and depth is assumed to improve the overall presence users experience by bringing the experience closer to real-world fidelity.

\subsubsection{System Performance}

An important factor in real-time, remote collaboration is maintaining minimal latency. The Insta360 Pro 2 was used as the 360-camera because of the combination of low latency (1.2s) and high visual quality. Even with a higher than ideal latency, participants were still satisfied with the system and even appreciated the overall low latency provided. During pilot testing, prior to the camera streaming optimisations, the camera latency was roughly 10 s and participants complained about the high latency. However, $1.2 \mathrm{~s}$ was low enough to feel real-time to users. Reasons for this could be because the video was not synced with the audio and interaction, giving the illusion that the latency was lower than it actually was. More research will need to be conducted to see if syncing latency across all mediums (visual, audio and interactions) makes the latency more noticeable and what is the threshold for noticeable latency in the system.

The rest of the system performed well during the study. The performance on the remote user side could decrease over time due to a bug in the code. The system was restarted prior to each study session to mitigate this problem. No other performance issues were noted during the study. The networking remained mostly stable during the evaluation and the bandwidth, although it can be further reduced with better optimisations, was adequate for the study. 


\subsubsection{Applications}

The strength of the system is in providing tele-presence and face-to-face communication when not all users can be physically present. This section discusses applications where the RC System can be beneficial and ideas for how such a setup can be achieved. Future modifications to better match the system with each application are also suggested.

Three possible applications for this system, as discussed by Rhee. et. al [88], include designing, remote assistance and training. The design task (e.g. interior design) allows users to mock-up a space and view how it looks in terms of lighting and aesthetics, similar to how users placed objects around the room during the two tasks. Major areas of improvements for making the system suitable for design tasks is in physical accuracy. As shown in Section 4.4.1, people were able to judge roughly where objects should be placed and rotated. However for an actual design task, the accuracy needs to be much higher. As already discussed, improved calibration between the users and better geometry reconstruction will result in higher accuracy. Additionally, further functionality should be added to allow for precise measurements instead of estimates. One user's feedback during the study suggested adding a tool for measuring distances in the space. Another issue noted during the study was people could become confused when matching the orientation of the blueprint to the physical space. A solution could be to automatically rotate the blueprint to account for the orientation of the user, similar to the needle in a compass.

The three applications suggested by Rhee. et. al [88] were prototyped using the RC System. The interior design was tested in task 1 of the user evaluation. The remote assistance and remote training was not evaluated, but was implemented as a proof of concept. The remote assistant scenario would see the remote user assist the local user in following a list of instructions. For the remote trainer scenario, the remote user can train, with the help of a digital replica, the local user to use physical equipment. The output for each application can be seen in Figure 4.8. 


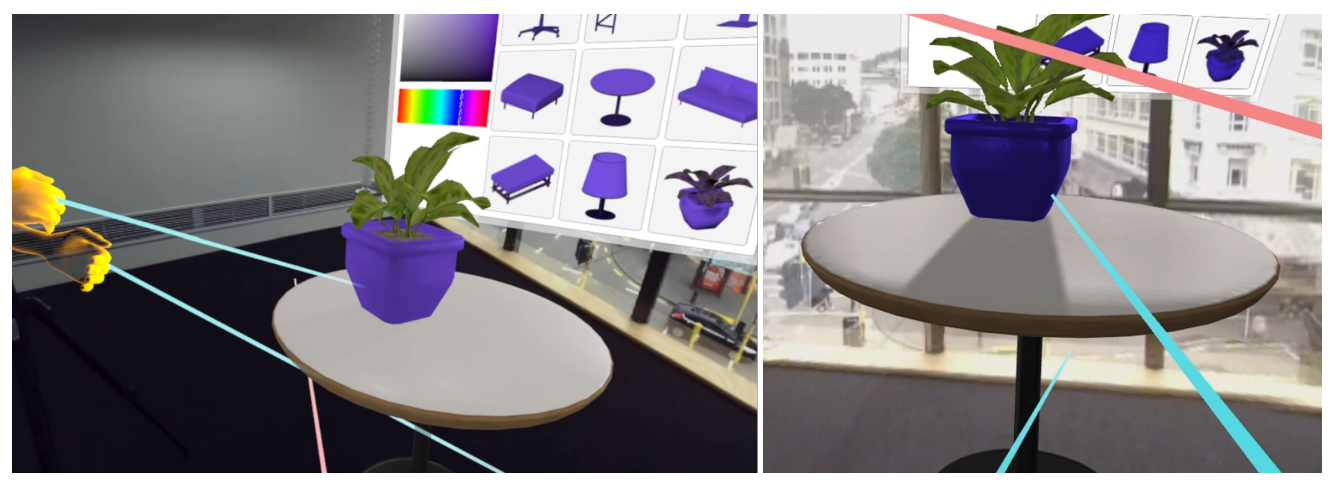

(a) Interior Design

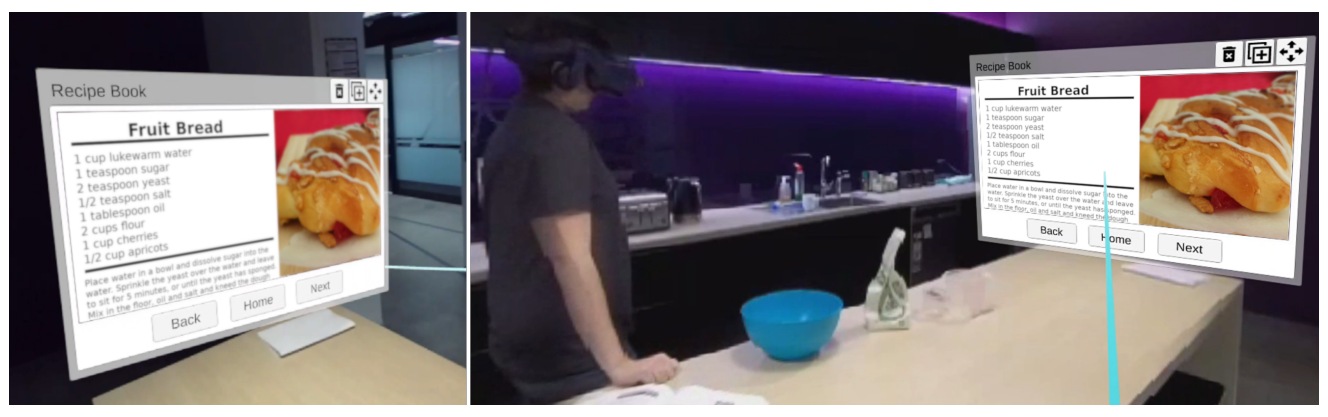

(b) Remote Assistance

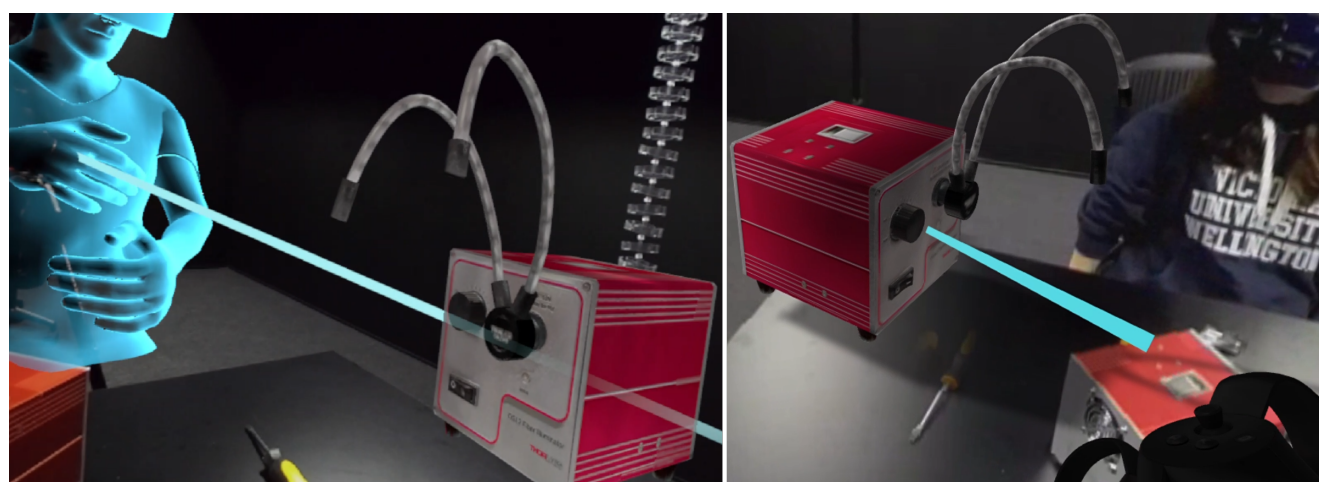

(c) Remote Training

Figure 4.8: Example applications as suggested by Rhee. et. al. [88]. The left images is the local user's view and the right images is the remote user's view. 


\section{Cinematic and Gaming Experiences}

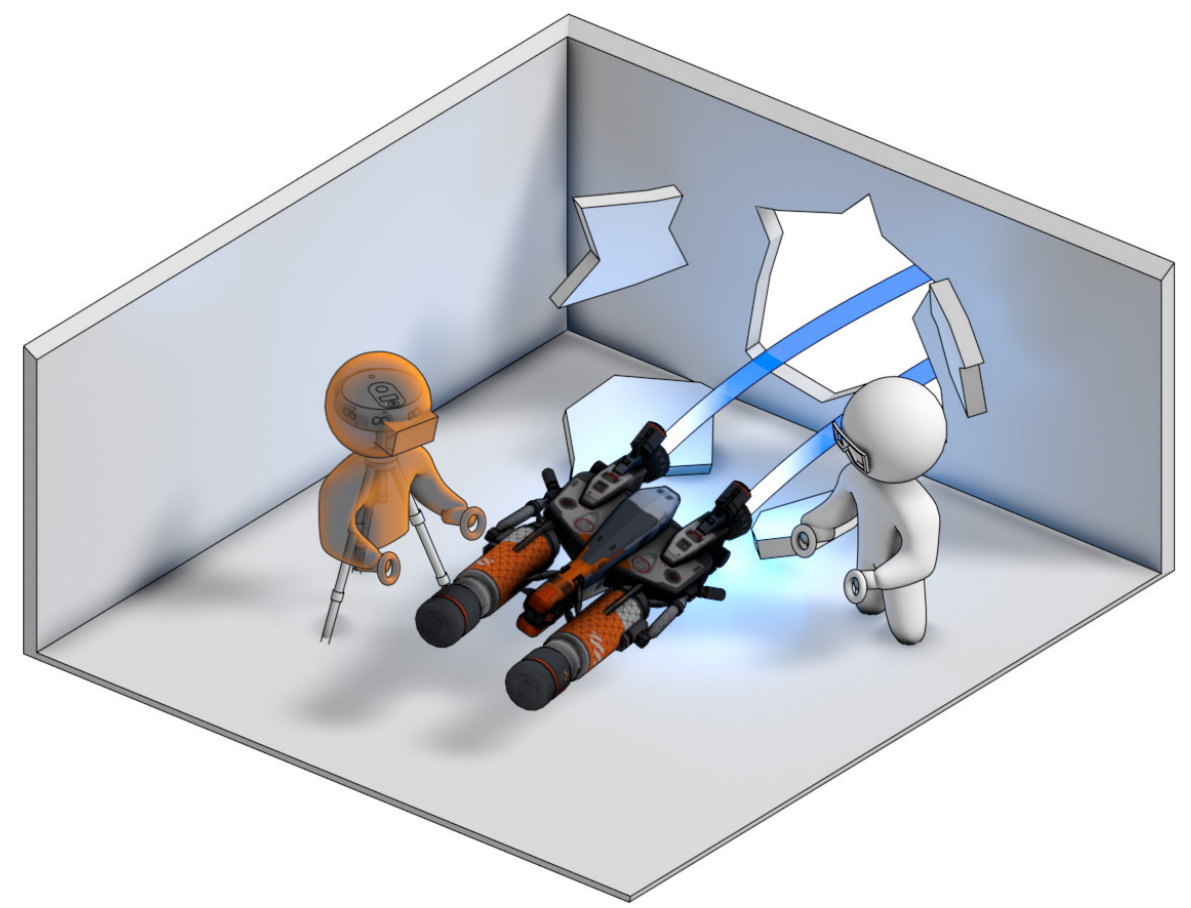

Figure 4.9: Two users experiencing a cinematic experience.

VR is commonly used to create gaming and, to a lesser degree, cinematic experiences. The introduction of AR HMDs has brought the augmentation of games into the real world. For example, objects in the game can detect and react to the real-world environment. A challenge to this new form of media is how to expand the experience to a shared experience for multiple users. In the case of local-to-local interaction, shared AR spaces are already possible with many AR HMD systems. However, because AR augments virtual objects into the real-world space, shared experiences will not work when users are located in different locations where the real-world environment appears different. 
One possible solution is for the AR user to create a virtual representation of the real-world space and send the geometry to the remote user to view in VR. However, this setup decreases visual fidelity and tele-presence by requiring the local user to be represented as an avatar and by having the scene represented as static, pre-baked geometry with textures. Using the RC System can help reduce these issues (Figure 4.9). Real-time lighting helps to ground the cinematic or gaming elements into the real-world, while users seeing each other helps to build an emotional connection during the experience. A limitation of this application, as already discussed, is that the remote user cannot move around the scene without further improvements to the system.

\section{Remote Presentation}

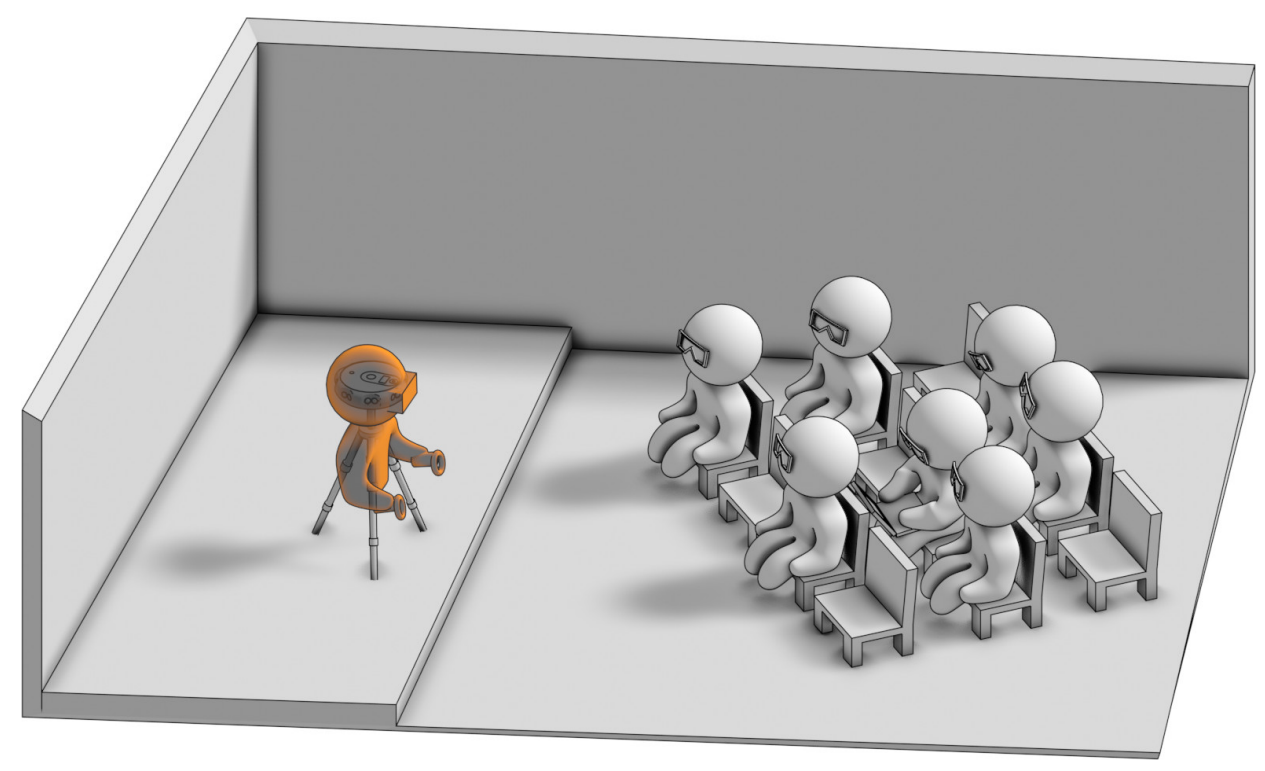

Figure 4.10: Remote User giving a presentation.

It is beneficial when presenting an engaging talk to an audience to have spatial awareness of the crowd and room. Current methods require remote presenters to join using a video call displayed on a large screen - a solu- 
tion that has many downsides. The remote presenter does not have a full field of view of the audience and cannot accurately judge and tailor the presentation to the audience. Likewise, the audience share reduced socialpresence with the presenter and will have less rapport compared to when the presenter is present.

The RC System addresses these issues by giving a complete $360^{\circ}$ view of the location to the presenter (Figure 4.10). Audiences will be able to clearly view the presenter represented through avatar form, providing the presenter with the freedom to use basic gestures to help aid their talk and to make eye contact with the audience for better engagement. The limitation to this idea is that the audience is required to use AR devices to view the presenter. A cost-effective solution would involve porting the system to mobile devices so that any audience member can download and use the system to participate in the talk. Another option, which requires no effort from the audience, is to project the avatar onto a human-sized screen on the stage. However, using this approach limits the ability to use virtual objects to enhance the presentation.

\subsubsection{Summary}

Although the RC System is still in a prototype stage, the overall response from participants was mainly positive in regards to user experience and system usability. Several users saw potential in the system as well as possible applications where the system could be invaluable. The visual quality had a mixed response, however the collaboration and communication aspects of the system were well received. The latency of the communication was low enough to not be noticed and participants were able to communicate and collaborate as if they were face-to-face. The primary goal of the system was to simulate this feeling of face-to-face interaction, such that all users felt present in the same, physical space. As has been shown and discussed, the RC System succeeded in achieving this goal. 


\section{Chapter 5}

\section{Conclusion}

\subsection{Summary}

This thesis presents a working prototype of a remote collaboration system. People situated in the same location or remotely can work collaboratively with each other as if they were all co-present in the same location. The RC System uses a live-streaming 360-camera displayed to the remote user to provide a high sense of tele-presence and social-presence. Users can interact with the same virtual objects and user interfaces simultaneously. The 360 -video is also used to render virtual objects with lighting that similarly matches the lighting present in the local space.

The RC System was evaluated to find how well it could provide collaboration, communication, social presence and spatial presence among users. The study found participants were satisfied with the communication, collaboration and high sense of presence offered by the system, as well as with the low latency of communication. The applications described by Rhee. et. al. [88] were expanded on using user feedback from the user study. Additional potential applications of shared cinematic experiences and remote presenting were also discussed. 


\subsection{Limitations}

Although the 360-video provides high presence for the remote user, it does present limitations in that the position of the 360-camera is fixed and only provides 3-DoF for rotation. In the RC System, the 360-video is projected onto 3D geometry as a way to provide a coarse sense of depth and limited parallax. However, this does not allow the remote user to move freely around the local space and moving too far away from the 360-video centre will create visible projection artefacts.

Another limitation comes from using the 360-camera as a representation of the remote user. Introducing more remote users will create multiple, intersecting avatars at the location of the 360-camera and may appear confusing to local users. Furthermore, remote users will not be able to partake in face-to-face communication with other remote users. This limits the number of remote users using the system in the current setup to only one.

Other limitations in the system are due to current technological limitations. The hardware for HMDs limit the amount of immersion the system can provide. The resolution of VR HMDs has increased since the release of the first generation of VR HMDs, but still has low enough resolution for pixels to appear noticeable. The 360-camera and AR see-through camera are also limited in resolution and fail to capture fine details. Another issue was latency. At 1.2s, the 360-video experienced higher than the ideal latency for real-time collaboration. Of this latency, half was spent recording and stitching the 360-video and the other half was spent encoding, streaming and decoding to the remote user. The video was not synced with the rest of the system to reduce the impact of latency on communication and collaboration. However, this causes problems with the audio and interactions appearing out of sync with the video. The limitations in latency and image resolution are beyond the scope of the system, but continual improvements in hardware will reduce the impact of these limitations. 


\subsection{Future Work}

In the current setup, the 360-video is streamed at the full resolution. This can be optimised to only stream data that is visible to the remote user. Related work has already explored this [96], and further exploration can help to reduce the bandwidth and computational requirements of the system. Furthermore, splitting the 360-video into two separate streams for viewing and lighting in a way that negligibly impacts latency should also be considered.

Additional improvements to the lighting can be considered for the local user. The exposure level of the AR see-through camera changes when viewing bright or dark locations. The lighting on the virtual objects does not adjust to match any changes in exposure, which can cause the virtual lighting to no longer match the brightness of the real-world lighting. Ideally, the lighting can automatically adapt for the see-through camera view.

Current space calibration between the local and remote users requires manual placement and rotation of a virtual representation of the 360-camera. AR devices detect points around the physical location and use these points as reference for inside-out tracking. Detecting corresponding trackingpoints in the 360-video is a possible solution to automate camera calibration. Alternatively, tracking devices have been attached to the 360-camera in related work to determine the position and orientation [84].

Once the system has been calibrated, the avatar for the remote user is displayed over the 360-camera as an abstract outline of a person. Feedback from the user evaluation suggests that users prefer seeing a closer representation of the remote user. Recent work has already explored generating a 3D reconstructed representation of people for avatar representation. Systems, including the Holoportation system [75], can display 3D reconstructions of the person and provide enough detail for remote users to communicate through body language. 
The user evaluation for the RC System focused on evaluating user presence [88], general user feedback and technical performance. Additional studies can be evaluated to capture greater detailed information addressing, but not limited to, user communication and how users perform when completing a wider variety of tasks within the system. 
Appendices 

Appendix A

System Instructions 


\section{Controller Input}

Vive Controller

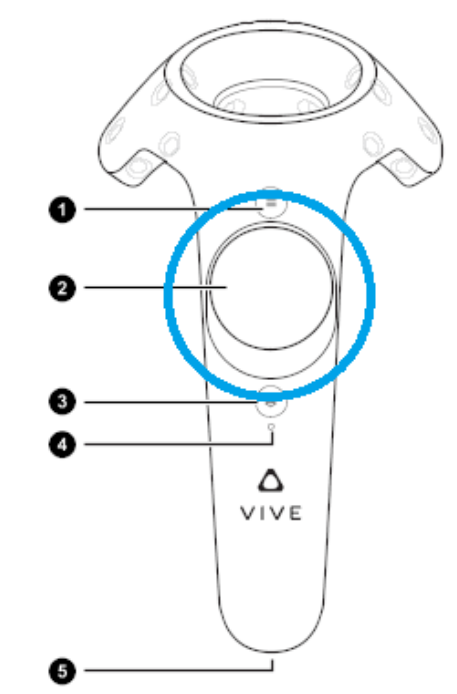

Action

Interact with Object (Orange)

Display Personal Menu (Blue)

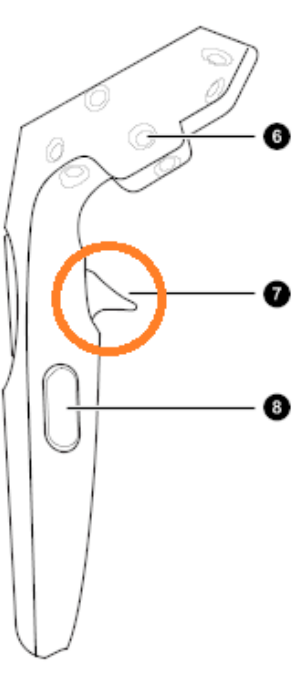

Vive

Trigger (7)

Thumb Button (2)
Oculus Controller

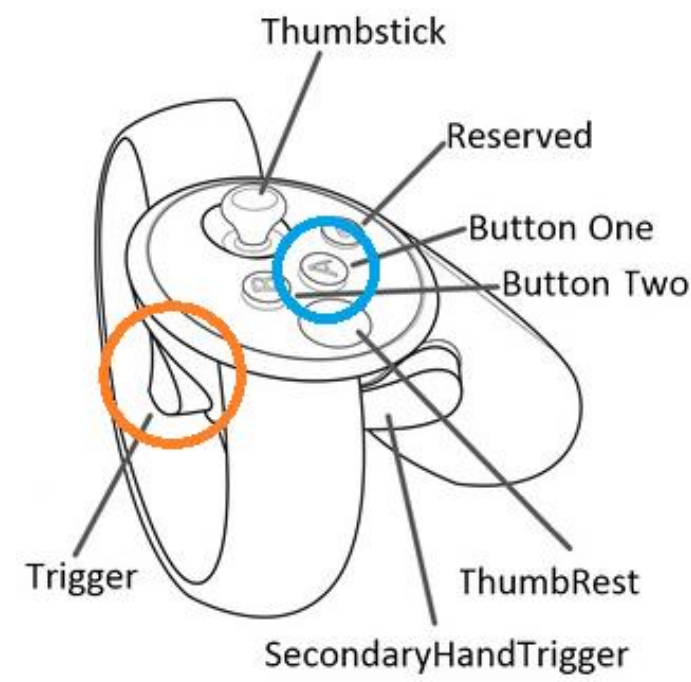

\section{Oculus}

Trigger

Button One

\section{Moving Objects}

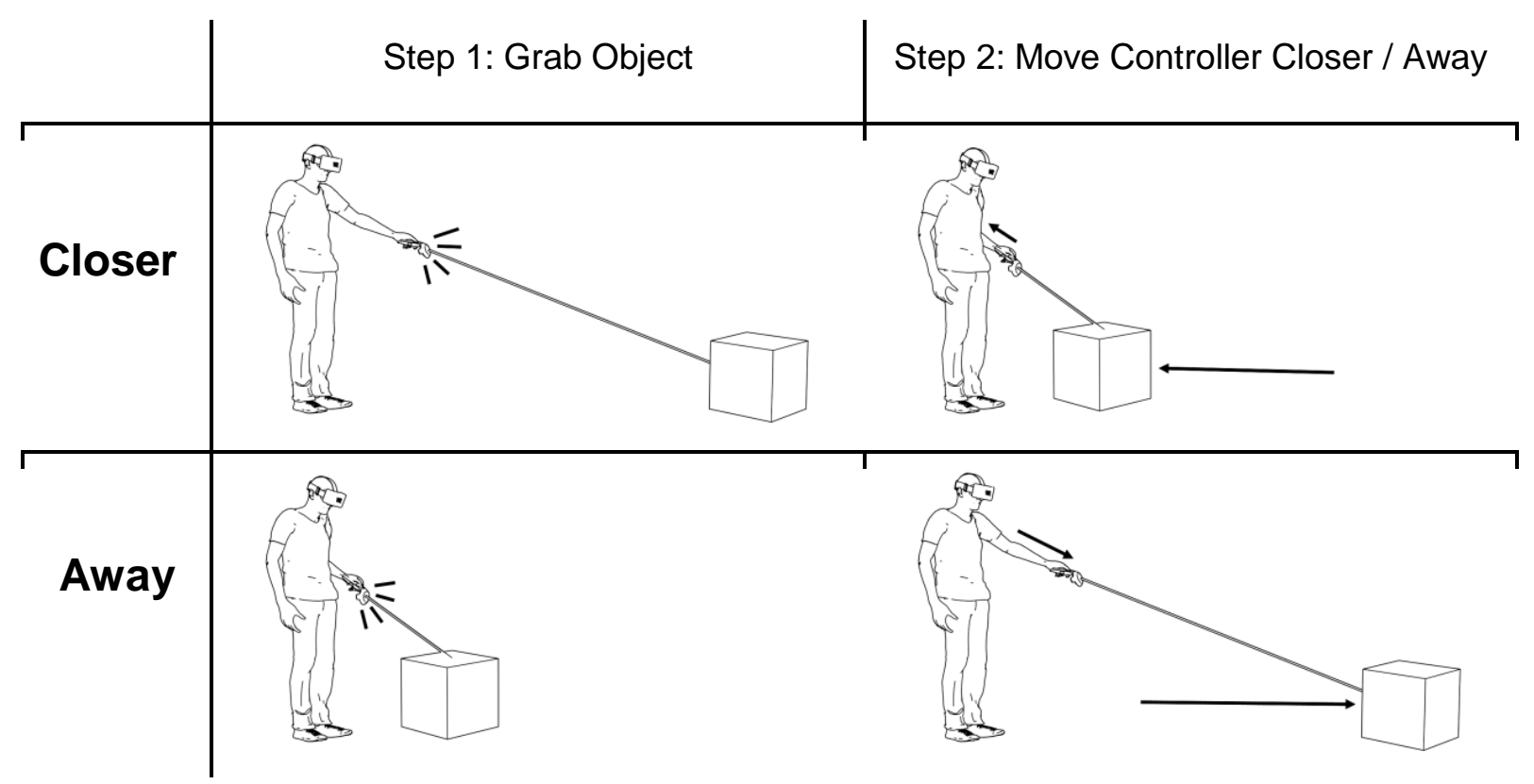




\section{Personal Menus}

Local User

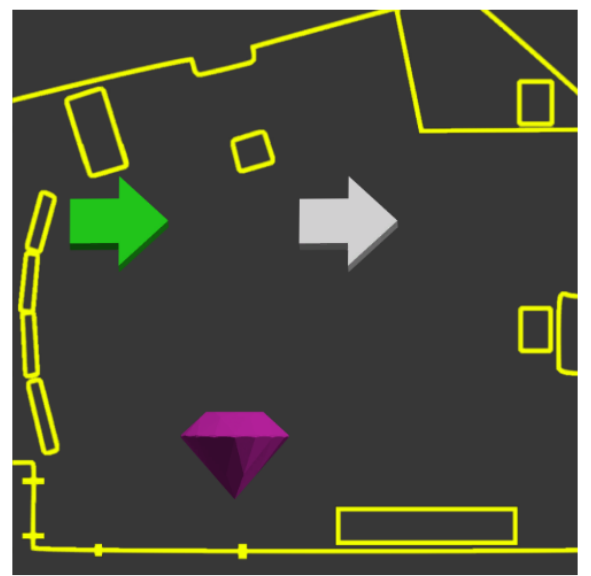

Recenter Avatar New Window

Calibrate Scene Spatial Mapping
Remote User

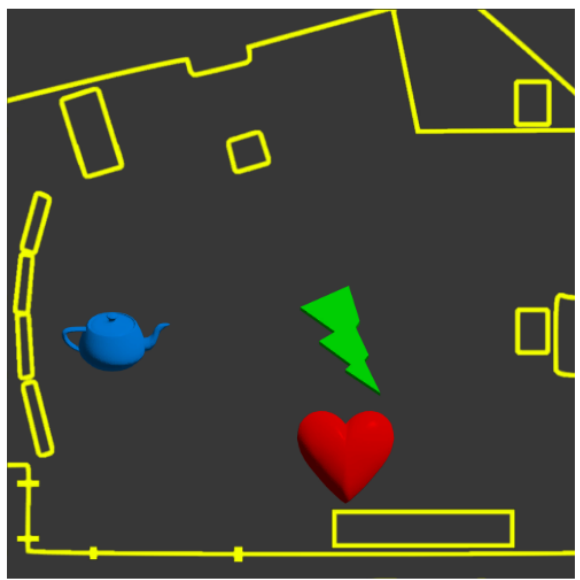

Turn Left

New Window

Turn Right

Change Avatar

Recenter View

\begin{tabular}{|c|l|}
\hline Button & \multicolumn{1}{c|}{ Action } \\
\hline New Window & Create a new shared menu window \\
\hline \multicolumn{2}{|c|}{ Local User } \\
\hline Recenter Avatar & Recenters the remote user avatar to the 360-camera \\
\hline Calibrate Scene & Sets the position of the camera, Ignore \\
\hline Spatial Mapping & Generates world geometry, Ignore \\
\hline \multicolumn{2}{|c|}{ Remote User } \\
\hline Turn Left/Right & Rotate view left or right \\
\hline Change Avatar & Modify the appearance of the remote avatar \\
\hline Recenter View & Realigns VR view to the 360-Video \\
\hline
\end{tabular}




\section{Shared Menus}

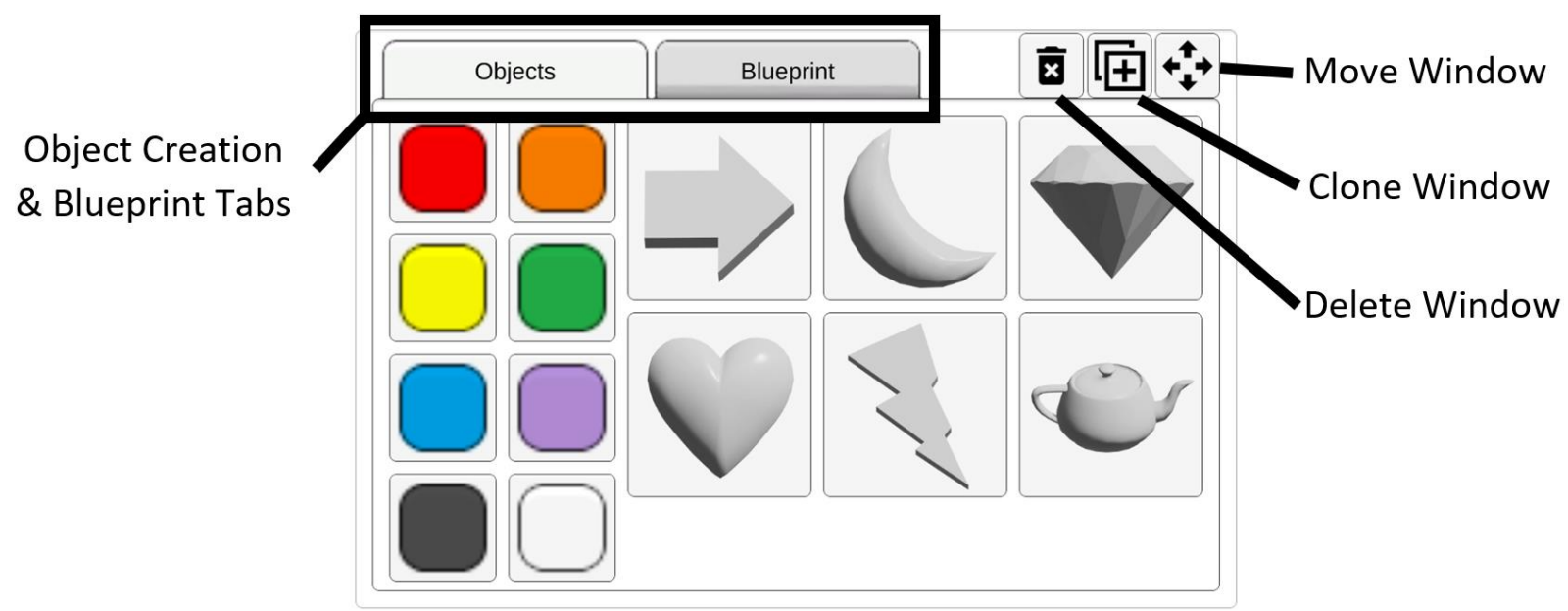

\section{Object Tab}

\section{Creating an Object}

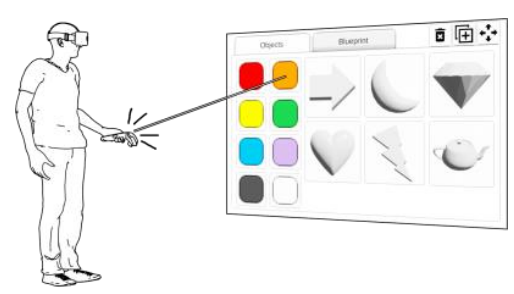

Step 1: Select a Colour

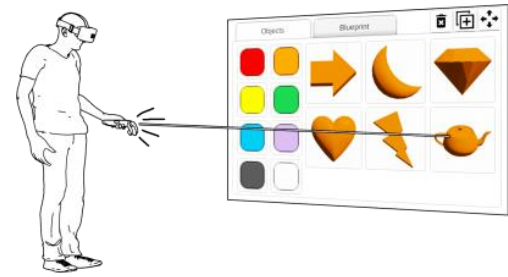

Step 2: Create an Object

Deleting an Object

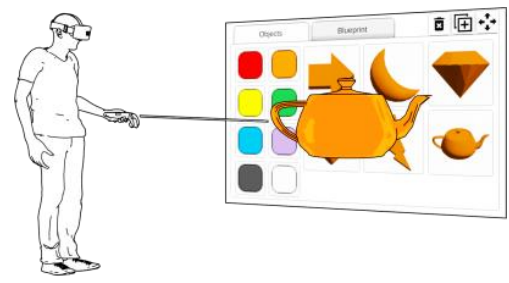

Step 3: Object Created!

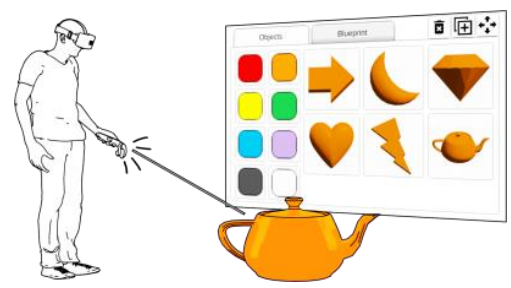

Step 1: Pick up object

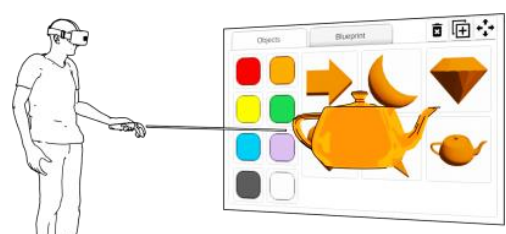

Step 2: Hover object over menu
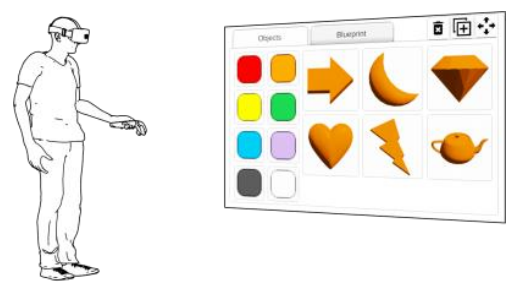

Step 3: Release trigger

Rotating an Object 


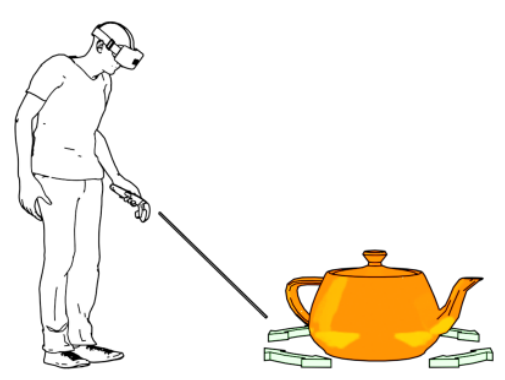

Step 1: Hover controller around object until arrows appear

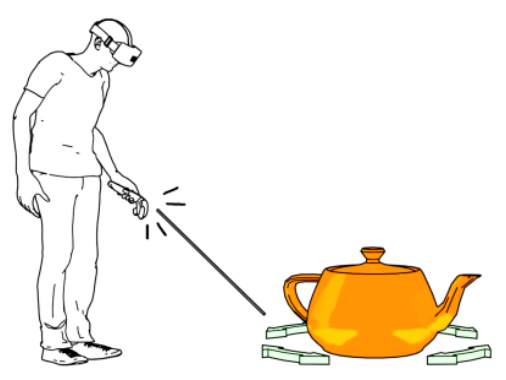

Step 2: Interact with arrows

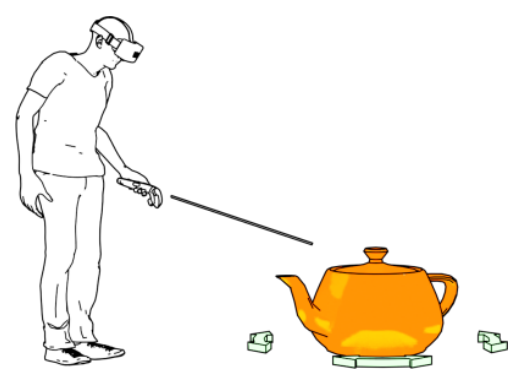

Step 3: Drag controller to rotate while holding trigger

\section{Blueprint Tab}

What the Local User Sees

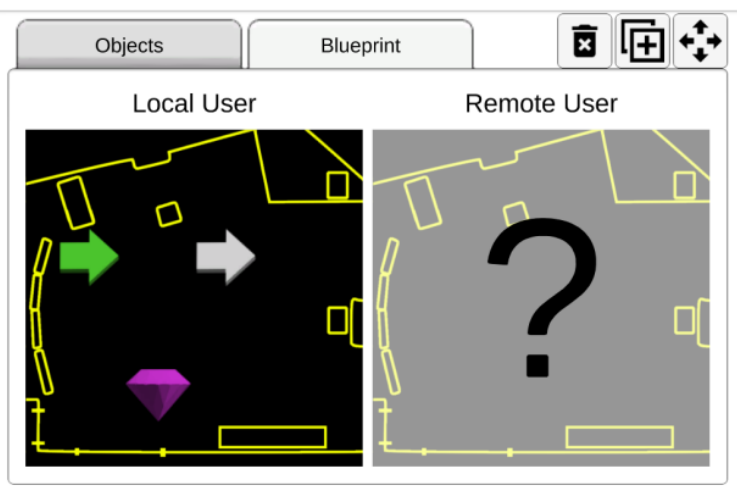

What the Remote User Sees

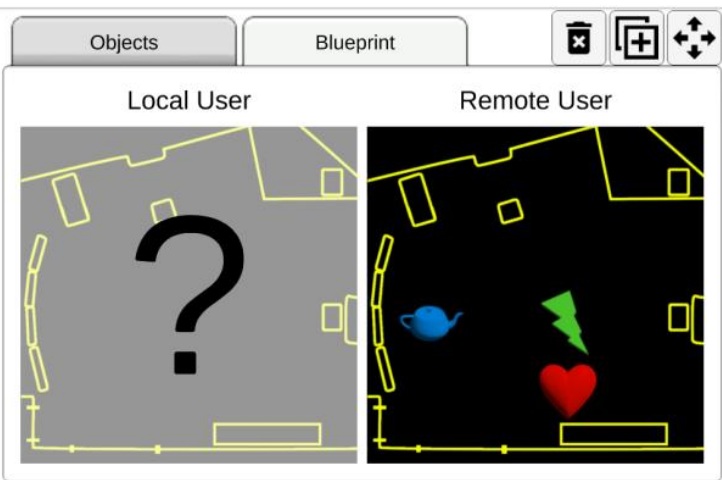

*Note: Users cannot see each other's blueprint during the study 
Appendix B

Consent Form 


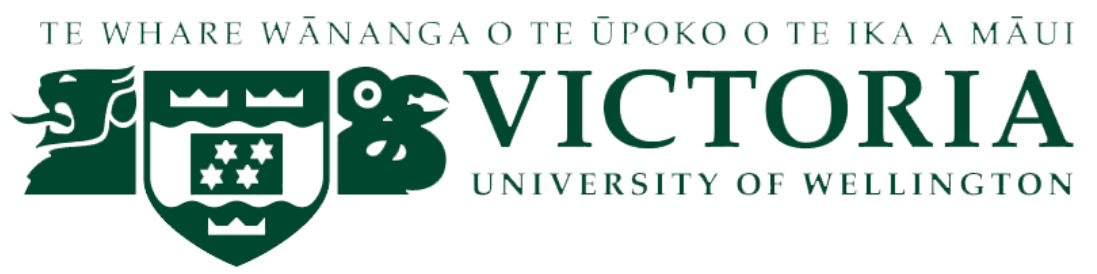

\section{Image/Video Quality and Experience Survey Participant Consent Form}

Researcher: Andrew Chalmers

School of Engineering and Computer Science, Victoria University of Wellington.

Please read the following notices. If you agree with them, please sign this form.

By signing this sheet: I, the participant, understand and agree that:

- My participation in this research is voluntary and I am aware that I am able to withdraw my participation and data at any time during my session, but not after the conclusion of my session

- I am aware that, should I choose to withdraw, that the investigator may ask me why I have chosen to withdraw but I do not have to provide any reasons if I do not want to.

- I am aware that physiological information will be collected, including eye-gaze, positional and head tracking.

- I am aware that my inputs when interacting with the system will be recorded, such as mouse movement, clicking, and screen capture (no other face or body information will be recorded).

- I confirm that I have have been provided, read and understand the Participant Information Sheet.

- I have had the opportunity to ask any questions about this research and had them answered.

- I understand that all personal information will remain confidential and that all efforts will be made to ensure I cannot be identified.

- I understand that the data I provide during my session will be used to produce aggregated data which will be held for 5 years.

- I agree that data gathered in this study will be stored anonymously and securely, and may be used for future research.

- I agree to take part in this study.

- If I have any further concerns and/or questions, I am aware I can use the following contacts: 
Participant Signature 
Appendix C

\section{Information Sheet}




\section{Image/Video Quality and Experience Survey Information Sheet}

Researcher: Andrew Chalmers

School of Engineering and Computer Science, Victoria University of Wellington. Application number: 0000026514

You are invited to take part in a study investigating image/video quality and experience. This study involves one session, during which you will view images or videos on a display (e.g. computer monitor, tablet, or head mounted display) and assess its visual quality or your experience/immersion when viewing/interacting with the images. There are five types of questions.

1. You will score an image's quality or your experience viewing the image with a score between 1 and 5, where each score has the following meaning:

1 Very bad

2 Bad

3 Neutral

4 Good

5 Very good

2. You will be shown pairs of images in which you will determine which image has better image quality.

3. You will be shown a set of images in which you will sort from best to worst based on their visual quality.

4. When viewing or interacting with this image/video (or in the case of a head mount display, the environment), how would you attribute your immersion?

1 Very low

2 Low

3 Neutral

4 High

5 Very high

5. You will be asked for your feedback and opinions as a participant. 
Physiological information will be collected, including eye-gaze, positional and head tracking.

Your inputs when interacting with the system will also be recorded. This may include mouse movement, clicking, and screen capture. No other face or body information will be recorded.

Each session will take a maximum of two hours (you may take breaks during the study). Data collected during this study will be stored in an anonymous form, in which no personally identifying information about you will be kept. You can choose either for your feedback response to be published in quotation, or for your sentiment to be reported without quotation. Otherwise, you can specify for your opinion to not be shared at all. You can withdraw participation and your data at any time during the session, but not after the conclusion of your session.

This Participant Information Sheet will help you decide if you would like to take part. It sets out why I am doing the study, what your participation would involve, what the benefits and risks to you might be, and what would happen after the study ends. Feel free to ask the researcher any questions.

If you agree to take part in this study, you will be asked to sign a consent form.

Please make sure you have read and understood both this Information Sheet and the Consent Form before signing.

This research has VUW Human Ethics Committee (HEC) approval (application number: 0000026514).

What are the conditions for my participation in this study? In

order to participate in this study, you must:

- Be over 18 years of age

- Able to view images on a screen or on paper

- Not have any serious visual abnormalities (amblyopia, spherical aberrations, etc.)

If you have a visual condition and are not sure if it will be an issue, please mention this to the researcher. Note that requiring prescription focal correction (i.e. long or short sightedness) is not an issue. If you are colour blind, you are still welcome to participate in the study, but you need to let the researcher know of your colour blind condition.

\section{Head mounted displays}

In order to participate in a study using a head mounted display, you must:

- Be over 18 years of age

- Not have a severe predisposition to motion sickness 
- Not have any serious visual abnormalities (amblyopia, spherical aberrations, etc.)

- Not have undergone eye surgery within the past year

- Not have a history of epileptic fits or other fits caused by visual stimuli

- Not be pregnant

The primary experimenter will be in the laboratory monitoring you at all times during the experiment. You are advised to stop the trial if you feel unwell. At the end of each run, we will ask you to report on any feelings of nausea or other adverse reaction.

With mild adverse reactions, you will be asked to sit quietly under the supervision of the researcher. Our experience is that feelings of nausea pass after a few minutes of sitting quietly and that subjects are then able to resume their daily routine. Subjects who experience a reaction will not be permitted to continue the experiment, even if they want to. Subjects who experience a mild reaction will be told to contact a medical practitioner if any symptoms persist into the following day.

For more serious reactions the researcher will use the telephone in the graphics research laboratory to contact Campus Safety on extension 8888, who will then call an appropriate First Aider.

In the unlikely event of an extreme reaction, the First Aider will advise on whether the subject should be released, sent home, sent to a doctor, or sent to hospital in an ambulance. In these cases, reasonable expenses (taxi, doctors fee) will be reimbursed to the subject from appropriate University funds (e.g., the supervisors research grant). An ambulance can be called from the telephone in the research lab, if necessary.

\section{What is the purpose of this study?}

To determine how well our algorithm works produce imagery in terms of image quality, performance or immersion.

\section{What will participating in this study involve?}

You will be invited to view a series of images for no more than two hours (you may take breaks during the study).

You will evaluate the quality of the image or video through a 1 to 5 scale, through pair-wise comparisons, by sorting a set of images from highest to lowest image quality, or by measuring your immersion when viewing the images.

Again: at any point, you are free to terminate the study. I will ask for a reason, but you do not have to provide one.

\section{What are the possible benefits and/or risks of this study?}


The results of this study will benefit the researchers by providing a way of assessing the researcher's algorithms. If the study uses a head mounted display, you will be informed beforehand. The risks are outlined above.

\section{What happens once this study ends?}

I will remove all identifying information about you from the results, and only store aggregated data: you may request from me these results, but they will not be tailored to you or mention you in any specific manner.

These aggregate results will be stored until 05/11/2023.

The aggregates results of this study will used for publications in academia in our upcoming and future research (thesis, conferences, journals).

\section{Compensation}

If your study is 1 hour or longer, you will be compensated with a coupon with a value of $\$ 20$.

\section{If you have any questions, who can you contact?}

\section{Redacted}

\section{Human Ethics Committee Information}

If you have any concerns about the ethical conduct of the research you may contact the Victoria University HEC Convenor:

\section{Redacted}

Thank you very much for your time and help in making this study possible. 
Appendix D

User Evaluation Questionnaire 


\section{User Evaluation Questionnaire}

\section{General Questions}

I felt that the virtual objects were situated in the same environment as the background.
Strongly Disagree
$\begin{array}{lll}1 & 2 & 3 \\ 0 & 0 & 0\end{array}$
$\begin{array}{llll}4 & 5 & 6 & 7\end{array}$
$0 \quad 0 \quad 0 \quad 0 \quad 00000$
Strongly Agree

It was easy to understand the other user when they were referring to an object or location

$\begin{array}{lllllllll} & 1 & 2 & 3 & 4 & 5 & 6 & 7 & \\ \text { Very Difficult } & 0 & 0 & 0 & 0 & 0 & 0 & 0 & \text { Very Easy }\end{array}$

It was easy to communicate using the voice

$\begin{array}{lllllllll} & 1 & 2 & 3 & 4 & 5 & 6 & 7 & \\ \text { Very Difficult } & \mathrm{O} & \mathrm{O} & \mathrm{O} & \mathrm{O} & \mathrm{O} & \mathrm{O} & \mathrm{O} & \text { Very Easy }\end{array}$

It was easy to communicate using visual elements (e.g. pointers, gestures)

$\begin{array}{lllllllll} & 1 & 2 & 3 & 4 & 5 & 6 & 7 & \\ \text { Very Difficult } & 0 & 0 & 0 & 0 & 0 & 0 & 0 & \text { Very Easy }\end{array}$

It was easy to create objects

$\begin{array}{lllllllll}\text { Very Difficult } & 1 & 2 & 3 & 4 & 5 & 6 & 7 & \\ & 0 & 0 & 0 & 0 & 0 & 0 & 0 & \text { Very Easy }\end{array}$

It was easy to select objects

$\begin{array}{lllllllll}\text { Very Difficult } & 1 & 2 & 3 & 4 & 5 & 6 & 7 & \\ & 0 & 0 & 0 & 0 & 0 & 0 & 0 & \text { Very Easy }\end{array}$

It was easy to move objects

$\begin{array}{lllllllll}\text { Very Difficult } & 1 & 2 & 3 & 4 & 5 & 6 & 7 & \\ & 0 & 0 & 0 & 0 & 0 & 0 & 0 & \text { Very Easy }\end{array}$

It was easy to perform the task 1 (furniture)

$\begin{array}{lllllllll} & 1 & 2 & 3 & 4 & 5 & 6 & 7 & \\ \text { Strongly Disagree } & 0 & 0 & 0 & 0 & 0 & 0 & 0 & \text { Strongly Agree }\end{array}$

It was easy to perform the task 2 (blueprint)

$\begin{array}{lllllllll} & 1 & 2 & 3 & 4 & 5 & 6 & 7 & \\ \text { Strongly Disagree } & 0 & 0 & 0 & 0 & 0 & 0 & 0 & \text { Strongly Agree }\end{array}$

It was easy to collaborate with the other user

$\begin{array}{lllllllll} & 1 & 2 & 3 & 4 & 5 & 6 & 7 & \\ \text { Strongly Disagree } & 0 & 0 & 0 & 0 & 0 & 0 & 0 & \text { Strongly Agree }\end{array}$


I felt that it was easy to collaborate with me

$\begin{array}{lllllllll} & 1 & 2 & 3 & 4 & 5 & 6 & 7 & \\ \text { Strongly Disagree } & 0 & 0 & 0 & 0 & 0 & 0 & 0 & \text { Strongly Agree }\end{array}$

I was active in solving the task 2 (blueprint)

$\begin{array}{lllllllll} & 1 & 2 & 3 & 4 & 5 & 6 & 7 & \\ \text { Strongly Disagree } & 0 & 0 & 0 & 0 & 0 & 0 & 0 & \text { Strongly Agree }\end{array}$

My partner was active in solving the task 2 (blueprint)

$\begin{array}{lllllllll} & 1 & 2 & 3 & 4 & 5 & 6 & 7 & \\ \text { Strongly Disagree } & 0 & 0 & 0 & 0 & 0 & 0 & 0 & \text { Strongly Agree }\end{array}$

I contributed on placing furniture (Please rate your level of contribution)

$\begin{array}{lllllllll} & 1 & 2 & 3 & 4 & 5 & 6 & 7 & \\ \text { Strongly Disagree } & 0 & 0 & 0 & 0 & 0 & 0 & 0 & \text { Strongly Agree }\end{array}$

My partner contributed on placing furniture (Please rate your partner's level of contribution)

$\begin{array}{lllllllll} & 1 & 2 & 3 & 4 & 5 & 6 & 7 & \\ \text { Strongly Disagree } & 0 & 0 & 0 & 0 & 0 & 0 & 0 & \text { Strongly Agree }\end{array}$

My partner talked more than me

$\begin{array}{lllllllll} & 1 & 2 & 3 & 4 & 5 & 6 & 7 & \\ \text { Strongly Disagree } & 0 & 0 & 0 & 0 & 0 & 0 & 0 & \text { Strongly Agree }\end{array}$

I talked more than my partner

$\begin{array}{lllllllll} & 1 & 2 & 3 & 4 & 5 & 6 & 7 & \\ \text { Strongly Disagree } & 0 & 0 & 0 & 0 & 0 & 0 & 0 & \text { Strongly Agree }\end{array}$

Were there any factors that made communication easier or harder?

What were some factors that helped or hindered your collaboration?

What were some factors that made you feel present with the other user? 
How would you improve the system?

\section{Self-Location}

I had the feeling that I was in the middle of the action rather than merely observing

$\begin{array}{lllllllll} & 1 & 2 & 3 & 4 & 5 & 6 & 7 & \\ \text { Strongly Disagree } & 0 & 0 & 0 & 0 & 0 & 0 & 0 & \text { Strongly Agree }\end{array}$

I felt like I was part of the environment in the presentation

$\begin{array}{lllllllll} & 1 & 2 & 3 & 4 & 5 & 6 & 7 & \\ \text { Strongly Disagree } & 0 & 0 & 0 & 0 & 0 & 0 & 0 & \text { Strongly Agree }\end{array}$

I felt like I was actually there in the environment

$\begin{array}{lllllllll} & 1 & 2 & 3 & 4 & 5 & 6 & 7 & \\ \text { Strongly Disagree } & 0 & 0 & 0 & 0 & 0 & 0 & 0 & \text { Strongly Agree }\end{array}$

I felt like the objects in the presentation surrounded me

$\begin{array}{lllllllll} & 1 & 2 & 3 & 4 & 5 & 6 & 7 & \\ \text { Strongly Disagree } & 0 & 0 & 0 & 0 & 0 & 0 & 0 & \text { Strongly Agree }\end{array}$

It was as though my true location had shifted into the environment in the presentation

$\begin{array}{lllllllll} & 1 & 2 & 3 & 4 & 5 & 6 & 7 & \\ \text { Strongly Disagree } & 0 & 0 & 0 & 0 & 0 & 0 & 0 & \text { Strongly Agree }\end{array}$

It seemed as though myself was present in the environment of the presentation

$\begin{array}{lllllllll} & 1 & 2 & 3 & 4 & 5 & 6 & 7 & \\ \text { Strongly Disagree } & 0 & 0 & 0 & 0 & 0 & 0 & 0 & \text { Strongly Agree }\end{array}$

I felt as though I was physically present in the environment of the presentation

$\begin{array}{lllllllll} & 1 & 2 & 3 & 4 & 5 & 6 & 7 & \\ \text { Strongly Disagree } & 0 & 0 & 0 & 0 & 0 & 0 & 0 & \text { Strongly Agree }\end{array}$

It seemed as though I actually took part in the action of the presentation

$\begin{array}{lllllllll} & 1 & 2 & 3 & 4 & 5 & 6 & 7 & \\ \text { Strongly Disagree } & \mathrm{O} & \mathrm{O} & \mathrm{O} & \mathrm{O} & \mathrm{O} & \mathrm{O} & \mathrm{O} & \text { Strongly Agree }\end{array}$

\section{Spatial Situation Model}

I was able to imagine the arrangement of the spaces presented in the headset very well

$\begin{array}{lllllllll} & 1 & 2 & 3 & 4 & 5 & 6 & 7 & \\ \text { Strongly Disagree } & \mathrm{O} & \mathrm{O} & \mathrm{O} & \mathrm{O} & \mathrm{O} & \mathrm{O} & \mathrm{O} & \text { Strongly Agree }\end{array}$


I had a precise idea of the spatial surroundings in the headset

$\begin{array}{lllllllll} & 1 & 2 & 3 & 4 & 5 & 6 & 7 & \\ \text { Strongly Disagree } & 0 & 0 & 0 & 0 & 0 & 0 & 0 & \text { Strongly Agree }\end{array}$

In my mind's eye, I was able to clearly see the arrangement of the objects presented/described

$\begin{array}{lllllllll} & 1 & 2 & 3 & 4 & 5 & 6 & 7 & \\ \text { Strongly Disagree } & 0 & 0 & 0 & 0 & 0 & 0 & 0 & \text { Strongly Agree }\end{array}$

I was able to make a good estimate of the size of the presented space

$\begin{array}{lllllllll} & 1 & 2 & 3 & 4 & 5 & 6 & 7 & \\ \text { Strongly Disagree } & 0 & 0 & 0 & 0 & 0 & 0 & 0 & \text { Strongly Agree }\end{array}$

I was able to make a good estimate of how far apart things were from each other

$\begin{array}{lllllllll} & 1 & 2 & 3 & 4 & 5 & 6 & 7 & \\ \text { Strongly Disagree } & 0 & 0 & 0 & 0 & 0 & 0 & 0 & \text { Strongly Agree }\end{array}$

Even now, I still have a concrete mental image of the spatial environment

$\begin{array}{lllllllll} & 1 & 2 & 3 & 4 & 5 & 6 & 7 & \\ \text { Strongly Disagree } & 0 & 0 & 0 & 0 & 0 & 0 & 0 & \text { Strongly Agree }\end{array}$

Even now, I could still draw a plan of the spatial environment in the presentation

$\begin{array}{lllllllll} & 1 & 2 & 3 & 4 & 5 & 6 & 7 & \\ \text { Strongly Disagree } & 0 & 0 & 0 & 0 & 0 & 0 & 0 & \text { Strongly Agree }\end{array}$

\section{Suspension of Disbelief}

I concentrated on whether there were any inconsistencies in the virtual objects of the presentation

$\begin{array}{lllllllll} & 1 & 2 & 3 & 4 & 5 & 6 & 7 & \\ \text { Strongly Agree } & \mathrm{O} & \mathrm{O} & \mathrm{O} & \mathrm{O} & \mathrm{O} & \mathrm{O} & \mathrm{O} & \text { Strongly Disagree }\end{array}$

I didn't really pay attention to the existence of errors or inconsistencies in the virtual objects of the presentation

$\begin{array}{lllllllll} & 1 & 2 & 3 & 4 & 5 & 6 & 7 & \\ \text { Strongly Disagree } & \mathrm{O} & \mathrm{O} & \mathrm{O} & \mathrm{O} & \mathrm{O} & \mathrm{O} & \mathrm{O} & \text { Strongly Agree }\end{array}$

I directed my attention to possible errors or contradictions in the virtual objects of the presentation

$\begin{array}{lllllllll} & 1 & 2 & 3 & 4 & 5 & 6 & 7 & \\ \text { Strongly Agree } & \mathrm{O} & \mathrm{O} & \mathrm{O} & \mathrm{O} & \mathrm{O} & \mathrm{O} & \mathrm{O} & \text { Strongly Disagree }\end{array}$

I thought about whether the action or the virtual objects of the presentation were plausible.
Strongly Agree

$12 \quad 2$
45
67

$\begin{array}{llllllll}0 & 0 & 0 & 0 & 0 & 0 & 0 & \text { Strongly Disagree }\end{array}$

I wondered whether the virtual objects' presentation could really exist like this.

$\begin{array}{lllllllll} & 1 & 2 & 3 & 4 & 5 & 6 & 7 & \\ \text { Strongly Agree } & \mathrm{O} & \mathrm{O} & \mathrm{O} & \mathrm{O} & \mathrm{O} & \mathrm{O} & \mathrm{O} & \text { Strongly Disagree }\end{array}$

I took a critical viewpoint of the virtual objects of the presentation.

$\begin{array}{lllllllll} & 1 & 2 & 3 & 4 & 5 & 6 & 7 & \\ \text { Strongly Agree } & \mathrm{O} & \mathrm{O} & \mathrm{O} & \mathrm{O} & \mathrm{O} & \mathrm{O} & \mathrm{O} & \text { Strongly Disagree }\end{array}$


It was important for me to check whether inconsistencies were present in the virtual objects.

$\begin{array}{lllllllll} & 1 & 2 & 3 & 4 & 5 & 6 & 7 & \\ \text { Strongly Agree } & \mathrm{O} & \mathrm{O} & \mathrm{O} & \mathrm{O} & \mathrm{O} & \mathrm{O} & \mathrm{O} & \text { Strongly Disagree }\end{array}$

It was not important for me whether the presentation contained errors or contradictions.

$\begin{array}{lllllllll} & 1 & 2 & 3 & 4 & 5 & 6 & 7 & \\ \text { Strongly Disagree } & 0 & 0 & 0 & 0 & 0 & 0 & 0 & \text { Strongly Agree }\end{array}$

\section{Social Presence Questionnaire}

I noticed my partner

$\begin{array}{lllllllll} & 1 & 2 & 3 & 4 & 5 & 6 & 7 & \\ \text { Fully Disagree } & 0 & 0 & 0 & 0 & 0 & 0 & 0 & \text { Fully Agree }\end{array}$

(My partner) noticed me

$\begin{array}{lllllllll} & 1 & 2 & 3 & 4 & 5 & 6 & 7 & \\ \text { Fully Disagree } & 0 & 0 & 0 & 0 & 0 & 0 & 0 & \text { Fully Agree }\end{array}$

(My partner's) presence was obvious to me

$\begin{array}{lllllllll} & 1 & 2 & 3 & 4 & 5 & 6 & 7 & \\ \text { Fully Disagree } & \mathrm{O} & \mathrm{O} & \mathrm{O} & \mathrm{O} & \mathrm{O} & \mathrm{O} & \mathrm{O} & \text { Fully Agree }\end{array}$

My presence was obvious to my partner

$\begin{array}{lllllllll} & 1 & 2 & 3 & 4 & 5 & 6 & 7 & \\ \text { Fully Disagree } & \mathrm{O} & \mathrm{O} & \mathrm{O} & \mathrm{O} & \mathrm{O} & \mathrm{O} & \mathrm{O} & \text { Fully Agree }\end{array}$

(My partner) caught my attention

$\begin{array}{lllllllll} & 1 & 2 & 3 & 4 & 5 & 6 & 7 & \\ \text { Fully Disagree } & \mathrm{O} & \mathrm{O} & \mathrm{O} & \mathrm{O} & \mathrm{O} & \mathrm{O} & \mathrm{O} & \text { Fully Agree }\end{array}$

I caught (my partner's) attention

$\begin{array}{lllllllll} & 1 & 2 & 3 & 4 & 5 & 6 & 7 & \\ \text { Fully Disagree } & \mathrm{O} & \mathrm{O} & \mathrm{O} & \mathrm{O} & \mathrm{O} & \mathrm{O} & \mathrm{O} & \text { Fully Agree }\end{array}$

I caught (my partner's) attention

$\begin{array}{lllllllll} & 1 & 2 & 3 & 4 & 5 & 6 & 7 & \\ \text { Fully Disagree } & \mathrm{O} & \mathrm{O} & \mathrm{O} & \mathrm{O} & \mathrm{O} & \mathrm{O} & \mathrm{O} & \text { Fully Agree }\end{array}$

\section{Attention Allocation}

I was easily distracted from (my partner) when other things were going on

$\begin{array}{lllllllll} & 1 & 2 & 3 & 4 & 5 & 6 & 7 & \\ \text { Fully Disagree } & 0 & 0 & 0 & 0 & 0 & 0 & 0 & \text { Fully Agree }\end{array}$

(My partner) was easily distracted from me when other things were going on
Fully Disagree
12
3
$4 \quad 5$
67
$0 \quad 0 \quad 0 \quad 0 \quad 0 \quad 000$
Fully Agree 
I remained focused on (my partner) throughout our interaction.

$\begin{array}{lllllllll} & 1 & 2 & 3 & 4 & 5 & 6 & 7 & \\ \text { Fully Disagree } & \mathrm{O} & \mathrm{O} & \mathrm{O} & \mathrm{O} & \mathrm{O} & \mathrm{O} & \mathrm{O} & \text { Fully Agree }\end{array}$

(My partner) remained focused on me throughout our interaction.

$\begin{array}{lllllllll} & 1 & 2 & 3 & 4 & 5 & 6 & 7 & \\ \text { Fully Disagree } & \mathrm{O} & \mathrm{O} & \mathrm{O} & \mathrm{O} & \mathrm{O} & \mathrm{O} & \mathrm{O} & \text { Fully Agree }\end{array}$

(My partner) did not receive my full attention.

$\begin{array}{lllllllll} & 1 & 2 & 3 & 4 & 5 & 6 & 7 & \\ \text { Fully Disagree } & \mathrm{O} & \mathrm{O} & \mathrm{O} & \mathrm{O} & \mathrm{O} & \mathrm{O} & \mathrm{O} & \text { Fully Agree }\end{array}$

I did not receive (my partner's) full attention.

$\begin{array}{lllllllll} & 1 & 2 & 3 & 4 & 5 & 6 & 7 & \\ \text { Fully Disagree } & \mathrm{O} & \mathrm{O} & \mathrm{O} & \mathrm{O} & \mathrm{O} & \mathrm{O} & \mathrm{O} & \text { Fully Agree }\end{array}$

\section{Perceived Message}

My thoughts were clear to (my partner).

$\begin{array}{lllllllll} & 1 & 2 & 3 & 4 & 5 & 6 & 7 & \\ \text { Fully Disagree } & 0 & 0 & 0 & 0 & 0 & 0 & 0 & \text { Fully Agree }\end{array}$

(My partner's) thoughts were clear to me.

$\begin{array}{lllllllll} & 1 & 2 & 3 & 4 & 5 & 6 & 7 & \\ \text { Fully Disagree } & \mathrm{O} & \mathrm{O} & \mathrm{O} & \mathrm{O} & \mathrm{O} & \mathrm{O} & \mathrm{O} & \text { Fully Agree }\end{array}$

It was easy to understand (my partner).

$\begin{array}{lllllllll} & 1 & 2 & 3 & 4 & 5 & 6 & 7 & \\ \text { Fully Disagree } & \mathrm{O} & \mathrm{O} & \mathrm{O} & \mathrm{O} & \mathrm{O} & \mathrm{O} & \mathrm{O} & \text { Fully Agree }\end{array}$

(My partner) found it easy to understand me.

$\begin{array}{lllllllll} & 1 & 2 & 3 & 4 & 5 & 6 & 7 & \\ \text { Fully Disagree } & \mathrm{O} & \mathrm{O} & \mathrm{O} & \mathrm{O} & \mathrm{O} & \mathrm{O} & \mathrm{O} & \text { Fully Agree }\end{array}$

Understanding (my partner) was difficult

$\begin{array}{lllllllll} & 1 & 2 & 3 & 4 & 5 & 6 & 7 & \\ \text { Fully Disagree } & 0 & 0 & 0 & 0 & 0 & 0 & 0 & \text { Fully Agree }\end{array}$

(My partner) had difficulty understanding me.
Fully Disagree
$\begin{array}{lll}1 & 2 & 3 \\ 0 & 0 & 0\end{array}$
$\begin{array}{llll}4 & 5 & 6 & 7\end{array}$
O 0000
Fully Agree 


\section{Bibliography}

[1] Pimax 8k vr headset pimax technology. https://pimaxvr.com/ products / 8k. (Accessed on 07/18/2019).

[2] Snapshot compression - gaffer on games. https: //webcache.googleusercontent.com/search?q=cache: EAM952JqE3sJ:https://gafferongames.com/post/ snapshot_compression $+\& c d=2 \& h l=e n \& c t=c l n k \& g l=n z$. (Accessed on 07/15/2019).

[3] Vive Pro AR (SRWorks) vs. ZED Mini Augmented Reality, Apr 2018. [Online; accessed 23. Jun. 2019].

[4] Hubs by Mozilla, Jul 2019. [Online; accessed 18. Jul. 2019].

[5] Microsoft HoloLens - Mixed Reality Technology for Business, Feb 2020. [Online; accessed 2. Feb. 2020].

[6] Plane - ARCore - Google Developers, Oct 2020. [Online; accessed 26. Oct. 2020].

[7] Tracking and Visualizing Planes - Apple Developer Documentation, Oct 2020. [Online; accessed 26. Oct. 2020].

[8] AdCock, M., Anderson, S., And Thomas, B. Remotefusion: real time depth camera fusion for remote collaboration on physical tasks. In Proceedings of the 12th ACM SIGGRAPH International Conference 
on Virtual-Reality Continuum and Its Applications in Industry (2013), ACM, pp. 235-242.

[9] Anton, D., Kurillo, G., And BAJCsy, R. User experience and interaction performance in $2 \mathrm{~d} / 3 \mathrm{~d}$ telecollaboration. Future Generation Computer Systems 82 (2018), 77-88.

[10] Aschenbrenner, D., Li, M., DukAlski, R., Verlinden, J., AND LUKOSCH, S. Exploration of different augmented reality visualizations for enhancing situation awareness for remote factory planning assistance. In Fourth IEEE VR International Workshop on 3D Collaborative Virtual Environ-1650 ments (3DCVE 2018) (2018).

[11] Bessa, M., Melo, M., NARciso, D., BArbosA, L., AND VASCONCElOS-RAPOSO, J. Does 3d 360 video enhance user's vr experience?: An evaluation study. In Proceedings of the XVII International Conference on Human Computer Interaction (2016), ACM, p. 16.

[12] Billinghurst, M., Nassani, A., And ReichHerzer, C. Social panoramas: using wearable computers to share experiences. In SIGGRAPH Asia 2014 Mobile Graphics and Interactive Applications (2014), ACM, p. 25.

[13] Bouchard, S., Dumoulin, S., Talbot, J., Ledoux, A.-A., Phillips, J., Monthuy-Blanc, J., Labonté-Chartrand, G., Robillard, G., Cantamesse, M., And Renaud, P. Manipulating subjective realism and its impact on presence: Preliminary results on feasibility and neuroanatomical correlates. Interacting with Computers 24, 4 (2012), 227-236.

[14] Brenton, H., Gillies, M., Ballin, D., And Chatting, D. The uncanny valley: does it exist and is it related to presence. Presence connect 8, 1 (2005). 
[15] BRIlakis, I., FATHI, H., AND RAshidi, A. Progressive 3d reconstruction of infrastructure with videogrammetry. Automation in Construction 20, 7 (2011), 884-895.

[16] BuLU, S. T. Place presence, social presence, co-presence, and satisfaction in virtual worlds. Computers $\mathcal{E}$ Education 58, 1 (2012), 154161.

[17] Buttussi, F., AND CHITTARO, L. Effects of different types of virtual reality display on presence and learning in a safety training scenario. IEEE transactions on visualization and computer graphics 24, 2 (2017), 1063-1076.

[18] CHEN, S. E. Quicktime vr: An image-based approach to virtual environment navigation. In Proceedings of the 22nd annual conference on Computer graphics and interactive techniques (1995), ACM, pp. 2938.

[19] Cidota, M., Lukosch, S., Datcu, D., And Lukosch, H. Comparing the effect of audio and visual notifications on workspace awareness using head-mounted displays for remote collaboration in augmented reality. Augmented Human Research 1, 1 (2016), 1.

[20] Clergeaud, D., Roo, J. S., Hachet, M., And Guitton, P. Towards seamless interaction between physical and virtual locations for asymmetric collaboration. In Proceedings of the 23rd ACM Symposium on Virtual Reality Software and Technology (2017), ACM, p. 17.

[21] Comeau, C. Headsight television system provides remote surveillance. Electronics (1961), 86-90.

[22] Debevec, P. Rendering synthetic objects into real scenes: Bridging traditional and image-based graphics with global illumination and high dynamic range photography. In Proceedings of the 25th annual 
conference on Computer graphics and interactive techniques (1998), ACM, pp. 189-198.

[23] Dourish, P., AND Bellotti, V. Awareness and coordination in shared workspaces. In CSCW (1992), vol. 92, pp. 107-114.

[24] DAlonzo, M., Mioli, A., FormicA, D., Vollero, L., AND Di PINO, G. Different level of virtualization of sight and touch produces the uncanny valley of avatars hand embodiment. Scientific reports 9, 1 (2019), 1-11.

[25] Elbamby, M. S., Perfecto, C., Bennis, M., And Doppler, K. Toward low-latency and ultra-reliable virtual reality. IEEE Network 32,2 (2018), 78-84.

[26] Ens, B., LAnir, J., TAng, A., Bateman, S., Lee, G., PiumsomBOON, T., AND BILLINGHURST, M. Revisiting collaboration through mixed reality: The evolution of groupware. International Journal of Human-Computer Studies (2019).

[27] FACEBOOK, I. 360 photos facebook 360 video. https: / / facebook360.fb.com/360-photos/. (Accessed on 06/18/2019).

[28] FONSECA, D., AND KRAUS, M. A comparison of head-mounted and hand-held displays for 360 videos with focus on attitude and behavior change. In Proceedings of the 20th International Academic Mindtrek Conference (2016), ACM, pp. 287-296.

[29] Fraustino, J. D., LeE, J. Y., Lee, S. Y., AND AhN, H. Effects of 360 video on attitudes toward disaster communication: Mediating and moderating roles of spatial presence and prior disaster media involvement. Public Relations Review (2018). 
[30] GaO, L., Bai, H., LeE, G., And Billinghurst, M. An oriented point-cloud view for $\mathrm{mr}$ remote collaboration. In SIGGRAPH ASIA 2016 Mobile Graphics and Interactive Applications (2016), ACM, p. 8.

[31] GaO, L., Bai, H., Lindeman, R., And Billinghurst, M. Static local environment capturing and sharing for $\mathrm{mr}$ remote collaboration. In SIGGRAPH Asia 2017 Mobile Graphics \& Interactive Applications (2017), ACM, p. 17.

[32] Gerathewohl, S. J. Fidelity of simulation and transfer of training: a review of the problem. Tech. rep., Civil Aerospace Medical Institute, 1969.

[33] Gerhard, M., Moore, D., And Hobbs, D. Embodiment and copresence in collaborative interfaces. International Journal of HumanComputer Studies 61, 4 (2004), 453-480.

[34] Google. Upload 360-degree videos - youtube help. https: //support.google.com/youtube/answer/6178631?hl=en. (Accessed on 06/18/2019).

[35] Gruber, L., Ventura, J., And Schmalstieg, D. Image-space illumination for augmented reality in dynamic environments. In Virtual Reality (VR), 2015 IEEE (2015), IEEE, pp. 127-134.

[36] HARMS, C., AND BIOCCA, F. Internal consistency and reliability of the networked minds measure of social presence.

[37] Heeter, C. Being there: The subjective experience of presence. Presence: Teleoperators \& Virtual Environments 1, 2 (1992), 262-271.

[38] Heidicker, P., Langbehn, E., And Steinicke, F. Influence of avatar appearance on presence in social vr. In 2017 IEEE Symposium on 3D User Interfaces (3DUI) (2017), IEEE, pp. 233-234. 
[39] Heilig, M. Beginnings: Sensorama and the telesphere mask. In Digital illusion (1998), ACM Press/Addison-Wesley Publishing Co., pp. 343-351.

[40] Higuch, K., Yonetani, R., And Sato, Y. Can eye help you?: Effects of visualizing eye fixations on remote collaboration scenarios for physical tasks. In Proceedings of the 2016 CHI Conference on Human Factors in Computing Systems (2016), ACM, pp. 5180-5190.

[41] Hosseini, M., And Swaminathan, V. Adaptive 360 vr video streaming: Divide and conquer. In 2016 IEEE International Symposium on Multimedia (ISM) (2016), IEEE, pp. 107-110.

[42] HuAnG, H.-C., AND Hung, Y.-P. Panoramic stereo imaging system with automatic disparity warping and seaming. Graphical Models and Image Processing 60, 3 (1998), 196-208.

[43] Huang, W., Billinghurst, M., Alem, L., And Kim, S. Handsintouch: sharing gestures in remote collaboration. In Proceedings of the 30th Australian Conference on Computer-Human Interaction (2018), ACM, pp. 396-400.

[44] Hvass, J. S., Larsen, O., Vendelbo, K. B., Nilsson, N. C., NorDAHL, R., AND SERAFIN, S. The effect of geometric realism on presence in a virtual reality game. In Virtual Reality (VR), 2017 IEEE (2017), IEEE, pp. 339-340.

[45] IEEE. The Effect of Avatar Appearance on Social Presence in an Augmented Reality Remote Collaboration (03 2019).

[46] INC, A. Altspacevr inc - be there, together. https://altvr. com/. (Accessed on 05/03/2019).

[47] IORNS, T., AND RHEE, T. Real-time image based lighting for 360degree panoramic video. In Pacific-Rim Symposium on Image and Video Technology (2015), Springer, pp. 139-151. 
[48] Johansen, R. Groupware: Computer support for business teams. The Free Press, 1988.

[49] KaJIYA, J. T. The rendering equation. In ACM Siggraph Computer Graphics (1986), vol. 20, ACM, pp. 143-150.

[50] KÁN, P., AND KaUfmanN, H. High-quality reflections, refractions, and caustics in augmented reality and their contribution to visual coherence. In Mixed and Augmented Reality (ISMAR), 2012 IEEE International Symposium on (2012), IEEE, pp. 99-108.

[51] Kasahara, S., Ando, M., Suganuma, K., and Rekimoto, J. Parallel eyes: Exploring human capability and behaviors with paralleled first person view sharing. In Proceedings of the 2016 CHI Conference on Human Factors in Computing Systems (2016), ACM, pp. 15611572 .

[52] Kasahara, S., AND ReKIMOto, J. Jackin head: immersive visual telepresence system with omnidirectional wearable camera for remote collaboration. In Proceedings of the 21st ACM Symposium on Virtual Reality Software and Technology (2015), ACM, pp. 217-225.

[53] Kim, D., Chae, S., SeO, J., YANG, Y., And Han, T.-D. Realtime plane detection for projection augmented reality in an unknown environment. In Acoustics, Speech and Signal Processing (ICASSP), 2017 IEEE International Conference on (2017), IEEE, pp. 5985-5989.

[54] Kim, S., Billinghurst, M., AND Lee, G. The effect of collaboration styles and view independence on video-mediated remote collaboration. Computer Supported Cooperative Work (CSCW) 27, 3-6 (2018), 569-607.

[55] Kishore, S., Navarro, X., Dominguez, E., De La Peña, N., AND SLATER, M. Beaming into the news: a system for and case 
study of tele-immersive journalism. IEEE computer graphics and applications (2016).

[56] Latoschik, M. E., Roth, D., Gall, D., Achenbach, J., WalteMATE, T., AND BOTSCH, M. The effect of avatar realism in immersive social virtual realities. In Proceedings of the 23rd ACM Symposium on Virtual Reality Software and Technology (2017), ACM, p. 39.

[57] LEAP, M. Home - magic leap. https : / /www . magicleap.com/. (Accessed on 05/01/2019).

[58] LEAP, M. Welcome to the shared reality - magic leap. https: / / creator.magicleap.com/learn/guides / leap-welcome-to-shared. (Accessed on 05/01/2019).

[59] LeE, G. A., TeO, T., Kim, S., And Billinghurst, M. A user study on $\mathrm{mr}$ remote collaboration using live 360 video. In 2018 IEEE International Symposium on Mixed and Augmented Reality (ISMAR) (2018), IEEE, pp. 153-164.

[60] LEE, H., HA, G., LEE, S., AND KIM, S. A mixed reality tele-presence platform to exchange emotion and sensory information based on mpeg-v standard. In Virtual Reality (VR), 2017 IEEE (2017), IEEE, pp. 349-350.

[61] Lensing, P., AND BROLL, W. Instant indirect illumination for dynamic mixed reality scenes. In Mixed and Augmented Reality (ISMAR), 2012 IEEE International Symposium on (2012), IEEE, pp. 109118.

[62] Lukosch, S., Lukosch, H., DAtCu, D., And Cidota, M. Providing information on the spot: Using augmented reality for situational awareness in the security domain. Computer Supported Cooperative Work (CSCW) 24, 6 (2015), 613-664. 
[63] MacQuarrie, A., AND Steed, A. Cinematic virtual reality: Evaluating the effect of display type on the viewing experience for panoramic video. In 2017 IEEE Virtual Reality (VR) (2017), IEEE, pp. $45-54$.

[64] McMahan, R. P., LaI, C., AND PAL, S. K. Interaction fidelity: the uncanny valley of virtual reality interactions. In International Conference on Virtual, Augmented and Mixed Reality (2016), Springer, pp. 59-70.

[65] Microsoft. Shared experiences in mixed reality mixed reality - microsoft docs. https://docs. microsoft.com/en-us/windows/mixed-reality/ shared-experiences-in-mixed-reality. (Accessed on 05/01/2019).

[66] Microsoft. What is a hologram? - Mixed Reality, June 2019. [Online; accessed 23. Jun. 2019].

[67] Milgram, P., AND Kishino, F. A taxonomy of mixed reality visual displays. IEICE TRANSACTIONS on Information and Systems 77, 12 (1994), 1321-1329.

[68] Milgram, P., Takemura, H., Utsumi, A., And Kishino, F. Augmented reality: A class of displays on the reality-virtuality continuum. In Telemanipulator and telepresence technologies (1995), vol. 2351, International Society for Optics and Photonics, pp. 282-293.

[69] Mori, M., MacDorman, K. F., AND KageKI, N. The uncanny valley [from the field]. IEEE Robotics Automation Magazine 19, 2 (2012), 98-100.

[70] Nagendran, A., Steed, A., Kelly, B., And Pan, Y. Symmetric telepresence using robotic humanoid surrogates. Computer Animation and Virtual Worlds 26, 3-4 (2015), 271-280. 
[71] NAIK, D., Curcio, I. D., AND TOUKOMAA, H. Optimized viewport dependent streaming of stereoscopic omnidirectional video. In Proceedings of the 23rd Packet Video Workshop (2018), ACM, pp. 37-42.

[72] NXRIGHTHERE. ENet-CSharp, Jan 2020. [Online; accessed 2. Feb. 2020].

[73] Oculus. Oculus homepage. https://www.oculus.com/. (Accessed on $05 / 01 / 2019)$.

[74] Oda, O., Elvezio, C., Sukan, M., Feiner, S., And Tversky, B. Virtual replicas for remote assistance in virtual and augmented reality. In Proceedings of the 28th Annual ACM Symposium on User Interface Software E Technology (2015), ACM, pp. 405-415.

[75] Orts-Escolano, S., Rhemann, C., FAnello, S., Chang, W., Kowdle, A., Degtyarev, Y., Kim, D., Davidson, P. L., Khamis, S., DOU, M., ET AL. Holoportation: Virtual 3d teleportation in realtime. In Proceedings of the 29th Annual Symposium on User Interface Software and Technology (2016), ACM, pp. 741-754.

[76] Otsuki, M., Maruyama, K., KuzuokA, H., And Suzuki, Y. Effects of enhanced gaze presentation on gaze leading in remote collaborative physical tasks. In Proceedings of the 2018 CHI Conference on Human Factors in Computing Systems (2018), ACM, p. 368.

[77] PAgAni, A., AND STRICKeR, D. Structure from motion using full spherical panoramic cameras. In 2011 IEEE International Conference on Computer Vision Workshops (ICCV Workshops) (2011), IEEE, pp. 375-382.

[78] Pardo, P. J., Suero, M. I., ANd PÉRez, Á. L. Correlation between perception of color, shadows, and surface textures and the realism of a scene in virtual reality. JOSA A 35, 4 (2018), B130-B135. 
[79] PARK, K. S., AND KenYON, R. V. Effects of network characteristics on human performance in a collaborative virtual environment. In Proceedings IEEE Virtual Reality (Cat. No. 99CB36316) (1999), IEEE, pp. 104-111.

[80] Pejsa, T., Kantor, J., Benko, H., Ofek, E., and Wilson, A. Room2room: Enabling life-size telepresence in a projected augmented reality environment. In Proceedings of the 19th ACM conference on computer-supported cooperative work \& social computing (2016), ACM, pp. 1716-1725.

[81] Peleg, S., Ben-Ezra, M., and Pritch, Y. Omnistereo: Panoramic stereo imaging. IEEE Transactions on Pattern Analysis \& Machine Intelligence, 3 (2001), 279-290.

[82] Pintaric, T., Neumann, U., And Rizzo, A. Immersive panoramic video. In Proceedings of the 8th ACM International Conference on Multimedia (2000), vol. 493.

[83] Piumsomboon, T., Lee, G. A., Hart, J. D., Ens, B., Lindeman, R. W., Thomas, B. H., AND Billinghurst, M. Mini-me: An adaptive avatar for mixed reality remote collaboration. In Proceedings of the 2018 CHI Conference on Human Factors in Computing Systems (2018), ACM, p. 46.

[84] Piumsomboon, T., Lee, G. A., Irlitti, A., Ens, B., Thomas, B. H., AND Billinghurst, M. On the shoulder of the giant: A multi-scale mixed reality collaboration with 360 video sharing and tangible interaction. In Proceedings of the 2019 CHI Conference on Human Factors in Computing Systems (2019), ACM, p. 228.

[85] RAmamoorthi, R., AND HANRAHAN, P. An efficient representation for irradiance environment maps. In Proceedings of the 28th an- 
nual conference on Computer graphics and interactive techniques (2001), ACM, pp. 497-500.

[86] Regenbrecht, H., Meng, K., Reepen, A., Beck, S., And LANGLOTZ, T. Mixed voxel reality: Presence and embodiment in low fidelity, visually coherent, mixed reality environments. In Mixed and Augmented Reality (ISMAR), 2017 IEEE International Symposium on (2017), IEEE, pp. 90-99.

[87] Rhee, T., Petikam, L., Allen, B., And Chalmers, A. Mr360: Mixed reality rendering for 360 panoramic videos. IEEE Transactions on Visualization E Computer Graphics (2017), 1379-1388.

[88] Rhee, T., Thompson, S., Medeiros, D., Anjos, R. D., AND CHALMERS, A. Augmented virtual teleportation for high-fidelity telecollaboration. In IEEE Transactions on Visualization $\mathcal{E}$ Computer Graphics (2020), IEEE.

[89] Robert, K., Zhu, D., HuAnG, W., Alem, L., And Gedeon, T. Mobilehelper: Remote guiding using smart mobile devices, hand gestures and augmented reality. In SIGGRAPH Asia 2013 Symposium on Mobile Graphics and Interactive Applications (2013), ACM, p. 39.

[90] Robinson, P., And Tuddenham, P. Distributed tabletops: Supporting remote and mixed-presence tabletop collaboration. In Second Annual IEEE International Workshop on Horizontal Interactive Human-Computer Systems (TABLETOP'07) (2007), IEEE, pp. 19-26.

[91] RoO, J. S., AND HACHET, M. Towards a hybrid space combining spatial augmented reality and virtual reality. In $3 D$ User Interfaces (3DUI), 2017 IEEE Symposium on (2017), IEEE, pp. 195-198.

[92] SCHMIDT, K. The problem withawareness': Introductory remarks onawareness in cscw'. Computer Supported Cooperative Work (CSCW) 11, 3-4 (2002), 285-298. 
[93] Schubert, T., Friedmann, F., And ReGEnbrecht, H. The experience of presence: Factor analytic insights. Presence: Teleoperators $\mathcal{E}$ Virtual Environments 10, 3 (2001), 266-281.

[94] Sheridan, T. B. Musings on telepresence and virtual presence. Presence: Teleoperators \& Virtual Environments 1, 1 (1992), 120-126.

[95] Sodhi, R. S., Jones, B. R., Forsyth, D., BAiley, B. P., AND MACIOCCI, G. Bethere: $3 \mathrm{~d}$ mobile collaboration with spatial input. In Proceedings of the SIGCHI Conference on Human Factors in Computing Systems (2013), ACM, pp. 179-188.

[96] SON, J., JANG, D., AND RYU, E.-S. Implementing 360 video tiled streaming system. In Proceedings of the 9th ACM Multimedia Systems Conference (2018), pp. 521-524.

[97] Speicher, M., CaO, J., Yu, A., Zhang, H., ANd Nebeling, M. 360anywhere: Mobile ad-hoc collaboration in any environment using 360 video and augmented reality. Proceedings of the ACM on Human-Computer Interaction 2, EICS (2018), 9.

[98] Sreedhar, K. K., Aminlou, A., Hannuksela, M. M., And GABвOUJ, M. Viewport-adaptive encoding and streaming of 360degree video for virtual reality applications. In 2016 IEEE International Symposium on Multimedia (ISM) (2016), IEEE, pp. 583-586.

[99] Steed, A., Steptoe, W., Oyekoya, W., Pece, F., Weyrich, T., Kautz, J., Friedman, D., Peer, A., Solazzi, M., Tecchia, F., ET AL. Beaming: an asymmetric telepresence system. IEEE computer graphics and applications 32, 6 (2012), 10-17.

[100] STEIN, J.-P., AND OHLER, P. Venturing into the uncanny valley of mindthe influence of mind attribution on the acceptance of humanlike characters in a virtual reality setting. Cognition 160 (2017), 43-50. 
[101] Steuer, J. Defining virtual reality: Dimensions determining telepresence. Journal of communication 42, 4 (1992), 73-93.

[102] Stotko, P., Krumpen, S., Hullin, M. B., Weinmann, M., AND KLEIN, R. Slamcast: Large-scale, real-time 3d reconstruction and streaming for immersive multi-client live telepresence. arXiv preprint arXiv:1805.03709 (2018).

[103] Sutherland, I. E. The ultimate display. Multimedia: From Wagner to virtual reality (1965), 506-508.

[104] Sutherland, I. E. A head-mounted three dimensional display. In Proceedings of the December 9-11, 1968, fall joint computer conference, part I (1968), ACM, pp. 757-764.

[105] TAIT, M., AND BillinghuRst, M. The effect of view independence in a collaborative ar system. Computer Supported Cooperative Work (CSCW) 24, 6 (Dec 2015), 563-589.

[106] Tang, A., Fakourfar, O., Neustaedter, C., And Bateman, S. Collaboration in 360 videochat: Challenges and opportunities. Tech. rep., University of Calgary, 2017.

[107] TARKO, J., TOMPKIN, J., AND RICHARDT, C. Omnimr: Omnidirectional mixed reality with spatially-varying environment reflections from moving 360 video cameras. In IEEE Conference on Virtual Reality and 3D User Interfaces (2019).

[108] TeO, T., LAwrence, L., LeE, G. A., Billinghurst, M., AND ADCOCK, M. Mixed reality remote collaboration combining 360 video and $3 \mathrm{~d}$ reconstruction. In Proceedings of the 2019 CHI Conference on Human Factors in Computing Systems (2019), ACM, p. 201.

[109] Tran, H. T., Ngoc, N. P., Pham, C. T., Jung, Y. J., And Thang, T. C. A subjective study on qoe of 360 video for vr communica- 
tion. In 2017 IEEE 19th International Workshop on Multimedia Signal Processing (MMSP) (2017), IEEE, pp. 1-6.

[110] Velamkayala, E. R., Zambrano, M. V., and Li, H. Effects of hololens in collaboration: A case in navigation tasks. In Proceedings of the Human Factors and Ergonomics Society Annual Meeting (2017), vol. 61, SAGE Publications Sage CA: Los Angeles, CA, pp. 21102114.

[111] ViveSoftware. ViveMediaDecoder, Feb 2019. [Online; accessed 2. Feb. 2020].

[112] VIVE. Vive new zealand - discover virtual reality beyond imagination. https://www.vive.com/nz/. (Accessed on 05/01/2019).

[113] Vorderer, P., Wirth, W., Gouveia, F. R., Biocca, F., SaAri, T., JÄNCKE, L., BÖCKING, S., SCHRAMM, H., GYSBERS, A., HARTMANN, T., ET AL. Mec spatial presence questionnaire. Retrieved Sept 18 (2004), 2015.

[114] Weech, S., Kenny, S., And Barnett-Cowan, M. Presence and cybersickness in virtual reality are negatively related: a review. Frontiers in psychology 10 (2019), 158.

[115] Wheatstone, C. Xviii. contributions to the physiology of vision.part the first. on some remarkable, and hitherto unobserved, phenomena of binocular vision. Philosophical transactions of the Royal Society of London, 128 (1838), 371-394.

[116] Zenati-Henda, N., Bellarbi, A., BenbelKacem, S., AND BelHOCINE, M. Augmented reality system based on hand gestures for remote maintenance. In 2014 International Conference on Multimedia Computing and Systems (ICMCS) (2014), IEEE, pp. 5-8.

[117] ZHAO, S. Toward a taxonomy of copresence. Presence: Teleoperators $\mathcal{E}$ Virtual Environments 12, 5 (2003), 445-455. 